\title{
A Family of Non-Abelian Kitaev Models on a Lattice: Topological Condensation and confinement
}

\author{
H. Bombin and M.A. Martin-Delgado \\ Departamento de Física Teórica I, Universidad Complutense, 28040. Madrid, Spain.
}

\begin{abstract}
We study a family of non-Abelian topological models in a lattice that arise by modifying the Kitaev model through the introduction of single-qudit terms. The effect of these terms amounts to a reduction of the discrete gauge symmetry with respect to the original systems, which corresponds to a generalized mechanism of explicit symmetry breaking. The topological order is either partially lost or completely destroyed throughout the various models. The new systems display condensation and confinement of the topological charges present in the standard non-Abelian Kitaev models, which we study in terms of ribbon operator algebras.
\end{abstract}

PACS numbers: 71.10.-w, 11.15.-q, 03.67.Pp, 71.27.+a

\section{INTRODUCTION}

The subject of topological orders poses new challenges in the understanding of new phases of matter due to novel effects in quantum many-body physics [1]. There is by now a good deal of examples in condensed matter, like in fractional Hall effect systems [2], [3] , [4], [5], [6], short range RVB (Resonating Valence Bond) models 7], 8], 9], 10]. or in quantum spin liquids [1], 12], 13], 5], 14], 15], 16], 17. There exists also exactly solvable models [18], 19], 20], 39], [40], that are paradigmatic examples for exhibiting topological properties that can be addressed in full detail since the whole spectrum of those models is known. Although topological orders typically arise in the quantum physics of two spatial dimensions, it is possible to construct exactly solvable models in three spatial dimensions and beyond [21]. There is yet another field in which topological orders appear naturally. It corresponds to discrete gauge theories that arise as a consequence of a spontaneous symmetry breaking mechanism from a continuous gauge group down to a discrete gauge group [22], 23], 24], 25], 26], 27], 28]. In these two-dimensional topological quantum field theories, the standard algebraic language to describe the residual gauge invariant properties of the excitations is that of quasitriangular Hopf algebras (quantum groups) [29].

At the same time, quantum systems with topological order provide new expectations for finding alternative ways of robust quantum computation [18], 30], 31]. In fact, there are several forms to set up schemes for topological quantum computation, some of them based on the braiding of quasiparticles [18], [32], 33], 34], 35], 36], [37], 38], some of them based solely on the topological entangled properties of the degenerate ground states, without selective addressing of the physical qubits and without resorting to braiding of excitations [39], [40], and others based on cluster states [41].

Topological orders can be thought of as new forms of long range entanglement and they are at the crossroads of condensed matter and quantum information [42], [43], [44], 45], [46], [47], 48], 449], [50]. Some forms of hidden topological orders in quantum spin chains can be detected with string order parameters, which in turn can be interpreted in the light of quantum information techniques, and their long-range entanglement detected with them [45] using matrix product states from condensed matter.

There are experimental proposals based on optical lattices [51], 52 to implement models with Abelian topological orders [53], and in particular, the study of the string order parameter mentioned above can also be proposed by means of these techniques [54]. There are also proposals for non-Abelian models based on Josephson junction arrays [55], 56], 57], in addition to the largely studied case of the fractional quantum Hall effect [38].

One of the emblematic examples of exactly solvable models to study topological orders on a lattice is the Kitaev model [18], both in its Abelian and non-Abelian versions. It captures the algebraic properties exhibited by the discrete gauge theories mentioned above. In addition, it provides us with an explicit realization of a Hamiltonian on a lattice, with the bonus that it allows for a model of topological quantum computation.

Comparatively, there are much less works on the nonAbelian Kitaev model than in the Abelian case (toric code). This is due, to some extent, to the additional mathematical technical difficulties presented by the nonAbelian case which is traditionally introduced with the language of quasi-triangular Hopf algebras and their representations [58], [59]. Here we have made an effort to explain its contents in full detail and clarity with simpler algebraic tools based on group theory and their representations. Our goal is twofold: to make the model more accessible to a broader audience with a previous knowledge on the Abelian toric code, and to use that simpler presentation as a starting point for considering more general models.

In this paper we introduce a family of non-Abelian topological models on a lattice, such that the standard Kitaev model corresponds to a particular case. More specifically, we study a two-parameter family labeled by a pair of subgroups $N \subset M \subset G, N$ normal in $G$, where $G$ is a discrete non-Abelian gauge group. The particular case $N=1, M=G$ correspond to the original 
Kitaev models. The Hamiltonians of the family, denoted $H_{G}^{N, M}$, are explicitly constructed in eq. (35). The standard vertex ('electric') operators are modified according to the subgroup $M$, while the face ('magnetic') operators change in accordance with $N$. In addition, there are new terms entering in the Hamiltonians which act on the edges of the lattice. Since there is a qudit attached to each edge these are single-qudit terms. Depending on the choice of the pair of subgroups $(M, N)$ with respect to $G$, the non-Abelian discrete gauge group of the whole Hamiltonian $H_{G}^{N, M}$ may range from $G$ down to the trivial group when $M=N$. This is so because the gauge group for these models turns out to be given by $G^{\prime}=M / N$. Therefore, the new family of non-Abelian models provides us with a mechanism of explicit symmetry breaking of an original Hamiltonian with large discrete gauge symmetry group. In other words, this mechanism can also be seen as a symmetry-reduction mechanism, since we may have still a smaller gauge symmetry present in the Hamiltonian.

The new edge terms do not commute with the vertex and face terms of the original Hamiltonian, but this can be compensated by slightly changing these vertex and face terms. This change corresponds to studying the regimen in which the single-qudit terms have a higher coupling constant. Choosing the models this way, we can study their ground state and also the charge condensation phenomena. At least in some cases, single-qudit terms can be understood as a mechanism for introducing string tension, or more appropriately 'ribbon tension', to some of the quasiparticle excitations which thus get confined. In those cases, a complete characterization of the charge types and domain wall fluxes will be given.

In order to facilitate both the exposition of the results and the readability of the manuscript, throughout the main text we will be giving the main constructions and results omitting many auxiliary details or proofs. However, all these can be found in a well-ordered form in a complete set of appendices.

We hereby summarize briefly some of our main results: i/ we introduce a family of Hamiltonians defined on twodimensional spatial lattices of arbitrary topology which exhibit a variety of discrete non-Abelian gauge group symmetry and topological orders;

ii/ the ground state of the models can be exactly given and characterized in terms of open a boundary ribbon operators. In many interesting cases the spectrum of excitations can be characterized accordingly;

iii/ the new models show condensation and confinement of the charges in the original models with Hamiltonian $H_{G}$;

iv/ in order to facilitate and complement the study of the family of models, we have carried out a thorough clarification of the main properties of the standard nonAbelian Kitaev model. In particular:

iv.a/ The ribbon operator algebra is introduced in an intrinsic way, with the motivation to find operators that describe excitations. iv.b/ We study in detail and generalize the concept of ribbon. In particular, closed ribbons and a related algebra are defined, and their transformation properties described.

iv.c/ The vertex and face operators that appear in the Hamiltonian are related to elementary closed ribbon operators, showing that everything in the models can be translated to the language of ribbons.

iv.d/ We give a detailed account of two-particle states, giving explicitly a basis for the states that clarifies the meaning of the labels for topological charge.

$\mathrm{v} /$ A description of the ground state in terms of boundary ribbon operators is given.

This paper is organized as follows: in Sect $\amalg$ we treat the standard non-abelian Kitaev model. We start explaining the terms appearing in the Hamiltonian and go on characterizing the ground state and quasiparticle excitations by means of closed ribbon operators. We also present an explicit characterization of the topological charges of the model and study when single-quasiparticle states are possible. In Sect III we motivate the new family of non-Abelian model Hamiltonians and present their generic properties. Then, we show how these models exhibit topological condensation and confinement described by domain walls. To this end we make use of closed and open ribbon operator algebras. Sect IV is devoted to conclusions.

Appendices deserve special attention since they contain the detailed and basic explanations of all the constructions used throughout the text. Specifically, Appendix A contains a brief summary of representation theory for group algebras, their centers and induced characters. In Appendix $B$ we perform an extensive treatment of ribbon operators, which are necessary to describe the whole spectrum of the models. We define ribbons as geometrical objects and then construct and characterize a series of ribbon operator algebras. In Appendix C we study the relationship between certain ribbon transformations and the action of ribbon operator algebras on suitable subspaces, which is a key ingredient in describing the topological properties of the models. In Appendix D we give some details about the local degrees of freedom that appear in the Hilbert space of two-particle excitations. In Appendix Ewe explain why single-quasiparticle states exist in non-abelian models on surfaces of nontrivial topology. Finally, in Appendix F we show several results needed for condensation and ground state characterization.

\section{NON-ABELIAN KITAEV MODEL}

\section{A. Hamiltonian}

The data necessary for building up the model, as introduced by Kitaev[18], are any given finite group $G$ and a lattice embedded in an orientable surface. The edges of the lattice must be oriented, as shown in Fig. 1. 


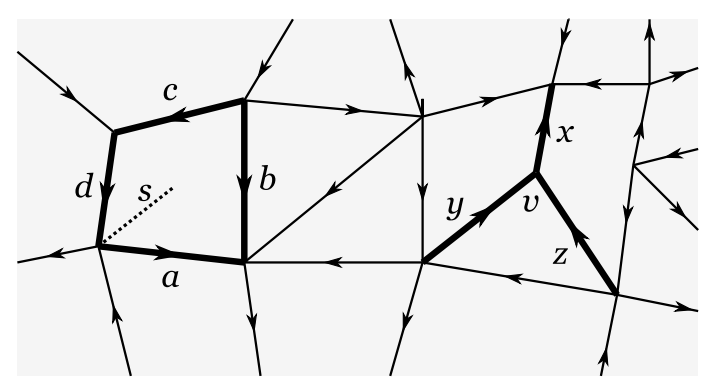

FIG. 1: The two dimensional lattice that we consider are arbitrary in shape and have oriented edges. Thick lines display the support of a face operator (left) and a vertex operator (right).

At every edge of the lattice we place a qudit, that is, a $|G|$-dimensional quantum system, with Hilbert space $\mathcal{H}_{G}^{\prime}$ with orthonormal basis $\{|g\rangle \mid g \in G\}$. This way, we identify $\mathcal{H}_{G}^{\prime}$ with the group algebra $\mathbf{C}[G]$. The Hilbert space for the whole system is then $\mathcal{H}_{G}:=\mathcal{H}_{G}^{\prime}{ }^{\otimes n}$, with $n$ the number of edges in the lattice. For notational convenience, we will denote the inverse of elements of $G$ as $\bar{g}$ instead of the usual $g^{-1}$. For completeness, we give a recollection of some basic properties of the group algebra $\mathbf{C}[G]$ in Appendix $\mathrm{A}$.

Usually, when we talk about sites in a lattice we mean its vertices. However, here we will say that a site $s$ is a pair $s=(v, f)$ with $f$ a face and $v$ one of its vertices [18]. The need to consider sites will be clarified later, when we discuss the excitations of the model in terms of strips associated to ribbon operators. This is in contrast with the Abelian case where one only needs to consider strings both in the direct and dual lattices. As it happens, to obtain a non-Abelian generalization we need to consider vertices and faces (plaquettes) in an unified manner through the concepts of sites, and strings and dual strings in an unified manner through the concept of ribbons.

The Hamiltonian of interest, as introduced in [18], is

$$
H_{G}=-\sum_{v} A_{v}-\sum_{f} B_{f}
$$

where the sums run over vertices $v$ and faces $f$. The terms $A_{v}$ and $B_{f}$ are projectors, called respectively vertex and face operators, or electric and magnetic operators. They commute with each other B37). In what follows, we give their explicit form.

First, we need a group of local operators at each vertex. We label its elements as $A_{v}^{g}, g \in G$, with $A_{v}^{g} A_{v}^{g \prime}=A_{v}^{g g \prime}$ so that they form a representation of $G$ on $\mathcal{H}_{G}$. The operators $A_{v}$ act only on those edges that meet at $v$, and this action depends on the orientation of the edge, inwards or outwards $v$. For example, for the vertex $v$ of figure (1) we have

$$
A_{v}^{g}|x, y, z, \cdots\rangle:=|g x, y \bar{g}, z \bar{g}, \cdots\rangle,
$$

where the dots represent other qudits, which do not change. These are the "local gauge transformation" 18] operators. The vertex operators $A_{v}$ that appear in the Hamiltonian are projectors onto the trivial sector of the representation of $G$ at $v$, that is

$$
A_{v}:=\frac{1}{|G|} \sum_{h \in G} A_{v}^{h}
$$

Now let $s=(v, f)$ be a site and $p_{s}$ denote the closed path with its endpoints in $v$ and running once and counterclockwise through the border of $f$. That is, $p_{s}$ is related to an elementary plaquette. We can then consider operators $B_{s}^{g}, g \in G$, that project onto those states with value $g$ for the 'product along $p_{s}$ '. For example, for the site $s$ of figure (1) we have

$$
B_{s}^{g}|a, b, c, d, \ldots\rangle:=\delta_{g, a \bar{b} c d}|a, b, c, d, \ldots\rangle .
$$

These are the "magnetic charge" [18] operators. Note that the orientation of the edges respect to the path is relevant. The face operators $B_{f}$ that appear in the Hamiltonian are projectors onto the trivial flux, that is

$$
B_{f}:=B_{s}^{1},
$$

where $s$ is any site with $s=(v, f)$ and 1 is the unit of $G$. The operator $B_{f}$ can be labeled just with the face, not with the particular site, because if the flux is trivial for a site then it is so for any other in the same face.

Since the Hamiltonian is a sum of projector operators, the ground state subspace contains those states $|\xi\rangle$ which are left invariant by the action of the vertex and face operators, namely,

$$
A_{v}|\xi\rangle=B_{f}|\xi\rangle=|\xi\rangle
$$

for every $v$ and $f$. That is, the projector onto the ground state is

$$
P_{\mathrm{GS}}=\prod_{v} A_{v} \prod_{f} B_{f} .
$$

In the sphere or the plane, there is no ground state degeneracy [18]. In particular, the ground state can be obtained easily

$$
\left|\psi_{G}\right\rangle=P_{\mathrm{GS}}|\mathbf{1}\rangle=\prod_{v} A_{v}|\mathbf{1}\rangle,
$$

where $|\mathbf{1}\rangle$ is the state with all the qudits in the state $|1\rangle$.

If an eigenstate violates some of the conditions (6) it is an excited state. Note that there is an energy gap from the ground state to excited states and that excitations are localized. If $A_{v}|\xi\rangle=0$, then we say that there is an electric quasiparticle at vertex $v$. If $B_{f}|\xi\rangle=0$, then we say that there is a magnetic quasiparticle at face $f$. In general electric and magnetic charges are interrelated, as we will see, and one says that quasiparticles are dyons that live at sites.

The excitations of these models carry topological charge. Let us explain what this means. First, consider a configuration with several excitations, far apart 
from each other. Each of these excitations has a type, a property that can be measured locally and does not change 18. It is this type what we refer as a topological charge. The point is that there exist certain degrees of freedom with a global, topological nature. In particular, there exists a subsystem which depends on the value of the charges and such that no local measurement is able to distinguish its states [18]. This subsystem is thus protected and a good place to store quantum information. When two quasiparticles get close, some degrees of freedom of the protected subsystem become local. This operation, called fusion, allows to perform measurements. Finally, one can perform unitary operations on the protected subsystem by suitably 'braiding' the excitations.

We will not be concerned with the particular rules that govern the processes of fusion and braiding. Instead, we only want to be able to label the topological charges. But for this, as we shall see, it is enough to study certain ribbon operator algebras, which are introduced next.

\section{B. Ribbon operators}

This section is devoted to ribbon operators [18], which will be extensively employed throughout the paper. The main motivation is that ribbon operators describe quasiparticle excitations above the ground state in the NonAbelian Kitaev model, much like string operators describe the corresponding excitations in the Abelian case. A full account of the properties and definitions for ribbon operators used in this section is presented in Appendix B. specially in B8 where a basic characterization theorem for ribbon operators is proven.

The basic idea behind ribbon operators is the following. First, ribbons are certain 'paths' that connect sites (not vertices), as shown in Fig. 2. Suppose that for every pair of sites $s$ and $s^{\prime}$ and for every ribbon $\rho$ connecting them we have at our disposal certain family of operators $\left\{O_{\rho}^{i}\right\}_{i}$ with support in the ribbon $\rho$. In particular, suppose that any state $|\psi\rangle$ with no excitations along $\rho$ except possibly at $s$ and $s^{\prime}$ can be written as

$$
|\psi\rangle=\sum_{i} O_{\rho}^{i}\left|\psi_{i}\right\rangle
$$

in terms of certain states $\left|\psi_{i}\right\rangle$ which have no excitations along $\rho$ except possibly at $s^{\prime}$, but not at $s$. Then, any state can be obtained from states with one excitation less by application of such ribbon operators. In the sphere, where as we will see there are no states with one excitation, this means that any configuration of excited sites can be obtained from the GS by application of ribbon operators connecting these sites. Thus, we are addressing a situation for quasiparticle excitations which clearly resembles that of the Abelian Kitaev model, where strings in the dual and direct lattice have operators attached to them that create excitations at their endpoints.

Before ribbons can be further considered, we need to give more structure to our lattice. In particular, we will

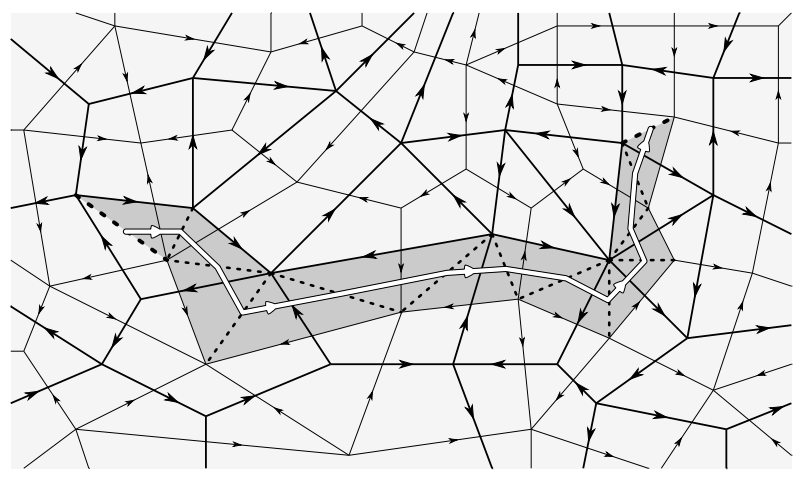

FIG. 2: Thick lines correspond to the lattice and thin lines to the dual lattice. Arrows show the orientation of edges and dual edges. Note that dual edges are oriented in agreement with edges (see explanation in main text). The shaded area is a ribbon. All the sites that form the ribbon are displayed as dashed lines, thicker in the case of the two sites in the ends. The arrowed thick white line shows the orientation of the ribbon.

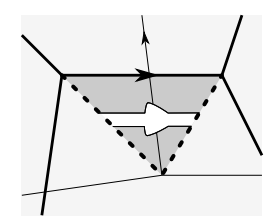

(a)

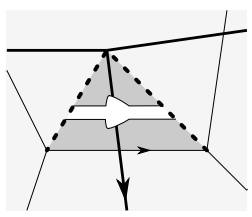

(c)

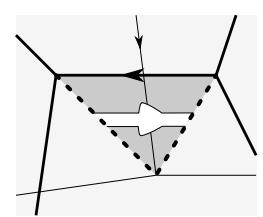

(b)

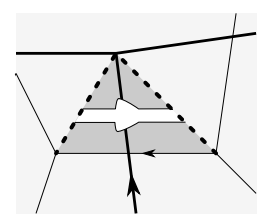

(d)
FIG. 3: Each figure represents a triangle $\tau$ (shaded area) that connects two sites (dashed lines): $\partial_{0} \tau$ to the left and $\partial_{1} \tau$ to the right. Thick lines correspond to the lattice and thin lines to the dual lattice. Arrows show the orientation of edges and dual edges. (a) A direct triangle with an edge which matches its direction. (b) A direct triangle with an edge which does not match its direction. (c) A dual triangle with a dual edge which matches its direction. (d) A dual triangle with a dual edge which does not match its direction.

have to deal with a 'merged' lattice in which the lattice and its dual play a simultaneous role. The reason to consider this merged lattice is that the excitations, as commented above, are related to sites, i.e., pairs $s=$ $(v, f)$ of a vertex and a face. Since the dual of a face is a vertex in the dual lattice, we could equally well say that a site is a pair of a vertex $v$ and a neighboring dual vertex $v^{\prime}=f^{*}$. Thus, a site is best visualized as a line connecting these two vertices, as the dashed lines shown in Fig. 2.

In order to have an oriented merged lattice, we orient the edges of the dual lattice in such a way that a dual 
edge $e^{*}$ crosses the edge $e$ 'from right to left', as in Fig. 2, This can be done because we are considering orientable surfaces only. Just as edges connect vertices in a normal lattice, we need something that connects sites in the merged lattice. These connectors turn out to be certain oriented triangles that come into two types: direct and dual triangles. A direct triangle $\tau$ is formed with two sites and an edge, as shown in Fig. 3( $(a, b)$. The idea is that $\tau$ points from a site $\partial_{0} \tau$ (dashed side to the left) to a site $\partial_{1} \tau$ (dashed side to the right) through an edge $e_{\tau}$ in the direct lattice. Note that the directions of $\tau$ and $e_{\tau}$ can either match or not, as the figure shows. A dual triangle $\tau^{\prime}$ is formed with two sites and a dual edge, see Fig. 3)(c,d). Again, it points from a site $\partial_{0} \tau^{\prime}$ to a site $\partial_{1} \tau^{\prime}$ through an edge $e_{\tau^{\prime}}^{*}$, which now belongs to the dual lattice. Again, the directions of $\tau^{\prime}$ and $e_{\tau^{\prime}}^{*}$ can either match or not, as the figure shows.

Just as in a usual lattice a list of composable edges forms a path, in the merged lattice a list of composable triangles forms a triangle strip. So a strip is a sequence of triangles $\rho=\left(\tau_{1}, \ldots, \tau_{n}\right)$ with the end of a triangle being the beginning of the next one, $\partial_{1} \tau_{i}=\partial_{0} \tau_{i+1}$. The ends of a strip are $\partial_{0} \rho=\partial_{0} \tau_{1}$ and $\partial_{1} \rho=\partial_{1} \tau_{n}$. A triangle strip is called a ribbon when it does not self-overlap, except possibly on its ends. A generic example of ribbon is shown in Fig. 2, For a detailed description of triangles, strips and ribbons on a lattice, we refer to Appendix B 1]

Our next task is to attach to each triangle an algebra of operators which is enough to move quasiparticles between its two ends, in the sense of (9). With this aim in mind, we first define triangle operators, which are single qudit operators acting on the edge $e_{\tau}$ of a triangle $\tau$. these operators depend on whether the triangle is direct or dual and on the relative orientation of $e_{\tau}$. The four possibilities are depicted in Fig. 3. The corresponding operators are
(a) $T_{\tau}^{g}|k\rangle=\delta_{g, k}|k\rangle$,
(b) $T_{\tau}^{g}|k\rangle=\delta_{\bar{g}, k}|k\rangle$,
(c) $L_{\tau}^{g}|k\rangle=|g k\rangle$,
(d) $L_{\tau}^{g}|k\rangle=|k \bar{g}\rangle$,

where $|k\rangle$ is the state of the qudit at the edge $e_{\tau}$. Thus, the triangle operators $T_{\tau}^{g}$ of direct triangles are projectors, like the $B_{s}^{g}$, and the triangle operators $L_{\tau}^{g}$ of dual triangles form a representation of $G$, like the $A_{v}^{g}$.

We start considering a direct triangle $\tau$. Since direct triangles connect sites with the same face but different vertices, triangle operators for direct triangles must be able to move electric, or vertex, excitations. Let $v, v^{\prime}$ be the two vertices of $\tau$. Then, as a special case of (B46),

$$
|G| \sum_{g \in G} T_{\tau}^{g} A_{v} T_{\tau}^{g}=1 .
$$

Thus, any state $|\psi\rangle$ can be expressed as

$$
|\psi\rangle=\sum_{g \in G} T_{\tau}^{g}\left|\psi_{g}\right\rangle
$$

with $\left|\psi_{g}\right\rangle=|G| A_{v} T_{\tau}^{g}|\psi\rangle$ an state with no excitation at $v$ because $A_{v}$ projects out electric excitations. Moreover,
$T_{\tau}^{g}$ commutes with all face operators and all vertex operators apart from those in the ends of $\tau$, so that $\left|\psi_{g}\right\rangle$ has no excited spots which are not already in $|\psi\rangle$, except possibly at $v^{\prime}$. These are the properties we were looking for and thus we define the algebra $\mathcal{A}_{\tau}$ as that with basis $\left\{T_{\tau}^{g}\right\}_{g \in G}$.

Next we consider a dual triangle $\tau$. Since dual triangles connect sites with the same vertex but different face, triangle operators for dual triangles must be able to move magnetic, or face, excitations. Let $f, f^{\prime}$ be the two faces of $\tau$. Then, as a special case of (B47),

$$
\sum_{g \in G} L_{\tau}^{\bar{g}} B_{f} L_{\tau}^{g}=1 .
$$

Thus, any state $|\psi\rangle$ can be expressed as

$$
|\psi\rangle=\sum_{g \in G} L_{\tau}^{\bar{g}}\left|\psi_{g}\right\rangle
$$

with $\left|\psi_{g}\right\rangle=B_{f} L_{\tau}^{g}|\psi\rangle$ an state with no excitation at $f$, because $B_{f}$ projects out magnetic excitations. Moreover, $L_{\tau}^{g}$ commutes with all vertex operators apart from those in the only vertex of $\tau$ and all face operators except those from the two faces connected by $\tau$, so that $\left|\psi_{g}\right\rangle$ has no excited sites which are not already in $|\psi\rangle$, except possibly at $f^{\prime}$. These are the properties we were looking for and thus we define the algebra $\mathcal{A}_{\tau}$ as that with basis $\left\{L_{\tau}^{g}\right\}_{g \in G}$.

Now that we have triangle operators at our disposal, we can move quasiparticles at will, in the sense of (9). In particular, if we want to move an excitation from one end of a ribbon $\rho=\left(\tau_{1}, \ldots, \tau_{n}\right)$ to the other end, we just proceed triangle by triangle. In other words, we can introduce an algebra $\mathcal{A}_{\rho}:=\bigotimes_{i} \mathcal{F}_{\tau_{i}}$ which contains a family of operators $\left\{O_{\rho}^{i}\right\}$ with the properties related to (9). $\mathcal{A}_{\rho}$ can be thought of as the algebra of all quasiparticle processes along $\rho$. Note that it is closed under the adjoint operator, $\mathcal{A}_{\rho}^{\dagger}=\mathcal{A}_{\rho}$.

However, if we are just interested in processes were no quasiparticles are created or destroyed but in the ends of $\rho$, as is the case for (9), then $\mathcal{A}_{\rho}$ is just too general. Instead, we consider the ribbon operator algebra $\mathcal{F}_{\rho} \subset$ $\mathcal{A}_{\rho}$, which contains those operators that do not create or destroy excitations along $\rho$. In other words $F \in \mathcal{F}_{\rho}$ if $\left[F, A_{v}\right]=\left[F, B_{f}\right]=0$ for any vertex $v$ and face $f$ which do not lie in the ends of $\rho$. Note that $\mathcal{F}_{\rho}$ is closed under the adjoint operator because $A_{v}=A_{v}^{\dagger}, B_{f}=B_{f}^{\dagger}$. These are the operators we were searching for in (9): a basis of $\mathcal{F}_{\rho}$ gives the desired operators $O_{\rho}^{i}$, see (B46, B47). $\mathcal{F}_{\rho}$ can be thought of as the algebra of processes in which a pair of quasiparticles is created in one end of the ribbon and then one of them is moved to the other end. In these terms, it is clear why excited states are expressible by means of ribbon operators acting on ground states.

A particularly meaningful basis for $\mathcal{F}_{\rho}$, explicitly given in (B66), consists of certain operators $F_{\rho}^{R C ; \mathbf{u v}}$, labeled by $C$, a conjugacy class of the group $G, R$, an irreducible representation of certain group $\mathbf{N}_{C}$ defined below, and the indices $\mathbf{u}=(i, j), \mathbf{v}=\left(i^{\prime}, j^{\prime}\right)$ with $i, i^{\prime}=1, \ldots,|C|$, 
$j, j^{\prime}=1, \ldots, n_{R}$. Here $|C|$ is the cardinality of $C$ and $n_{R}$ is the degree of the representation $R$. The group $\mathbf{N}_{C}$ is defined as that with elements $g \in G$ with $g r_{C}=r_{C} g$ for some chosen representative $r_{C} \in C$. In order to construct

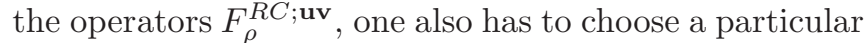
unitary matrix representation $\Gamma_{R}$ for $R$ and enumerate the elements of the conjugacy class as $C=\left\{c_{i}\right\}$, together with a suitable subset $\left\{q_{i}\right\}_{i=1}^{|C|} \subset G$ such that $c_{i}=q_{i} r_{C} \bar{q}_{i}$. Later we will relate the labels $R, C$ to the topological charges of the model and show how the indices $\mathbf{u}, \mathbf{v}$ are related to local degrees of freedom at both ends of the ribbon. We will use the following notation to denote linear combinations of ribbon operators with the same topological charge label $R, C$

$$
F_{\rho}^{R C}(\boldsymbol{\alpha}):=\sum_{\mathbf{u}, \mathbf{v}} \alpha^{\mathbf{u}, \mathbf{v}} F_{\rho}^{R C ; \mathbf{u v}},
$$

where $\alpha^{\mathbf{u v}} \in \mathbf{C}$.

In the case of abelian groups there are no local degrees of freedom and the elements of the basis are $F_{\rho}^{R C}=F_{\rho}^{\chi, g}$ with $g \in G$ and $\chi$ an element of the character group of $G$. These operators are unitary and form a group:

$$
F_{\rho}^{\chi, g} F_{\rho}^{\chi^{\prime}, g^{\prime}}=F_{\rho}^{\chi \chi^{\prime}, g g^{\prime}}, \quad F_{\rho}^{\chi, g \dagger}=F_{\rho}^{\bar{\chi}, \bar{g}} .
$$

Indeed, $T_{\rho}^{\chi}:=F_{\rho}^{\chi, 1}$ are the string operators of abelian models, and $L_{\rho}^{g}:=F_{\rho}^{e, g}$ the co-string operators, with $e$ the identity character.

An essential property of ribbon operators, which reflects the topological nature of the model, is that in the absence of excitations the particular shape of the ribbon is unimportant: We can deform the ribbon while keeping the action of the ribbon operator invariant. More exactly, if the state $|\psi\rangle$ is such that the ribbon $\rho$ can be deformed, with its ends fixed, to obtain another ribbon $\rho^{\prime}$ without crossing any excitation, then

$$
F_{\rho}^{R C}(\boldsymbol{\alpha})|\psi\rangle=F_{\rho^{\prime}}^{R C}(\boldsymbol{\alpha})|\psi\rangle .
$$

This is illustrated in Fig. 4.

\section{Closed ribbons}

For a closed ribbon $\sigma$ we mean one for which both ends coincide, so that we can set $\partial \sigma:=\partial_{0} \sigma=\partial_{1} \sigma$. In view of the definition of $\mathcal{F}_{\rho}$, in the case of closed ribbons it is natural to consider a subalgebra $\mathcal{K}_{\sigma} \subset \mathcal{A}_{\sigma}$ such that it forgets the single end $\partial \sigma$. With this goal in mind, we let $\mathcal{K}_{\sigma} \subset \mathcal{A}_{\sigma}$ contain those operators in $\mathcal{A}_{\sigma}$ that commute with all vertex and face operators $A_{v}, B_{f}$. In terms of quasiparticle processes, such closed ribbon operators are related to processes in which a pair of quasiparticles is created and one end of them is moved along the ribbon till they meet again to fuse into vacuum. Closed ribbon operators play a fundamental role in characterizing the ground state of the model in a similar fashion as how closed strings are the building blocks for the ground state

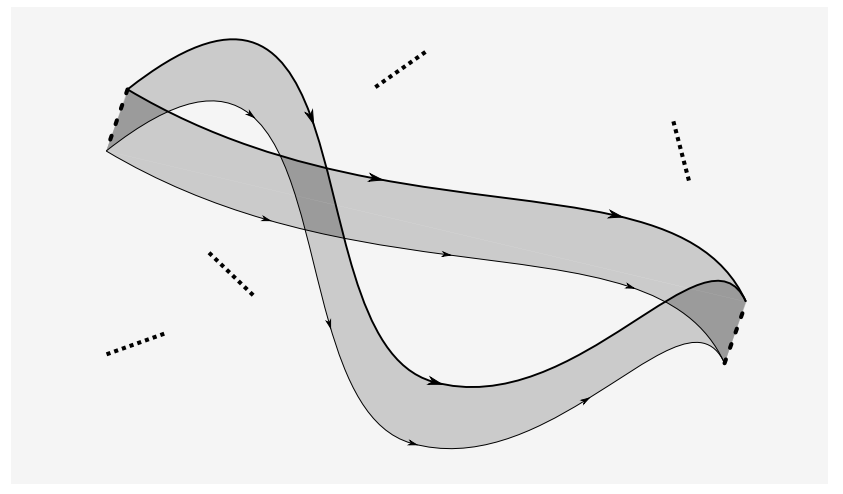

FIG. 4: An example of a deformation of a ribbon. The endpoints are fixed, and the area in between the two ribbons does not contain any excited site, which are represented with dotted lines.

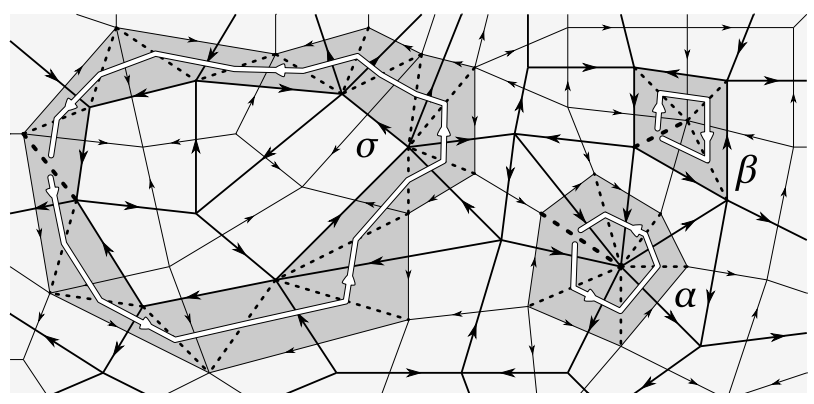

FIG. 5: Three examples of closed ribbons. $\sigma$ is a proper closed ribbon, containing both dual and direct triangles. It is also a boundary ribbon, as it encloses an area with the topology of a disc. $\alpha$ is a dual closed ribbon and thus encloses a single vertex. $\beta$ is a direct closed ribbon and thus encloses a single face.

in the Abelian case (toric code). A detailed analysis of closed ribbon operators is performed in Appendix B9.

We first consider the smallest examples of closed ribbons, i.e., dual and direct closed ribbons. We say that a ribbon is direct (dual) if it consists only of direct (dual) triangles. A dual ribbon like $\alpha$ in Fig. 5 encloses a single vertex $v$, and $\mathcal{K}_{\alpha}$ has as basis the operators $A_{v}^{h}, h \in G$. A direct ribbon like $\beta$ in Fig. [5 encloses a single face $f$, and $\mathcal{K}_{\beta}$ has as basis the operators $B_{f}^{C}$. These are labeled by the conjugacy classes $C$ of $G$ and take the form $B_{s}^{C}=\sum_{g \in C} B_{s}^{g}$ for any $s=(v, f)$. Thus, after defining ribbon operators by means of vertex and face operators, we now see that vertex and face operators are themselves ribbon operators.

As for the rest of closed ribbons $\sigma$, which we call proper closed ribbons, it turns out that $\mathcal{K}_{\sigma}$ has as basis certain orthogonal projectors $K_{\sigma}^{R C}$ that form a resolution of the identity, as shown in proposition 9. The labels $R, C$ of these projectors are the same appearing in the basis for $\mathcal{F}_{\rho}$. In fact, in the next section we will characterize exci- 


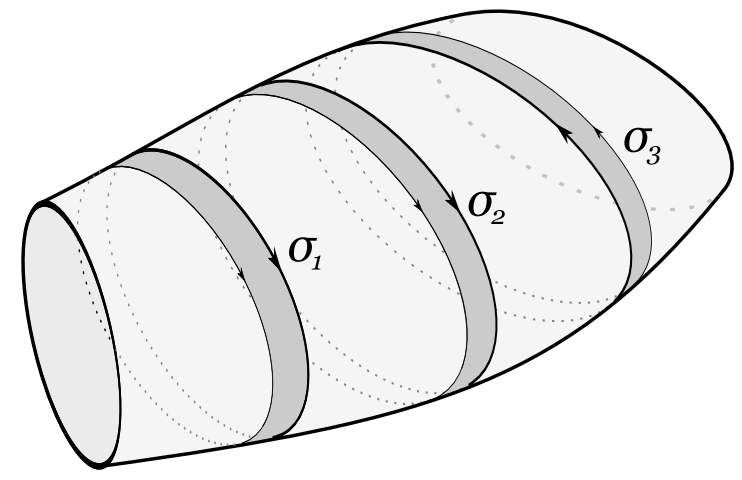

FIG. 6: Examples of closed ribbon transformations. A tubular piece of surface is displayed. The closed ribbon $\sigma_{1}$ is a deformation of $\sigma_{2}$ as long as there are no excitations between them. The ribbon $\sigma_{3}$ has an inverse orientation, and thus to obtain it from $\sigma_{2}$ we have to consider a deformation plus an inversion.

tations in terms of closed ribbon operators.

The algebra $\mathcal{K}_{\sigma}$ does not see the ends of $\sigma$. Because of this, unlike $\mathcal{F}_{\sigma}$, it can stand deformations in which the end $\partial \sigma$ is not fixed or, for that matter, rotations of the ribbon. More exactly, if the state $|\psi\rangle$ is such that the closed ribbon $\sigma$ can be deformed to obtain another ribbon $\sigma^{\prime}$ without crossing any excitation then

$$
K_{\sigma}^{R C}|\psi\rangle=K_{\sigma^{\prime}}^{R C}|\psi\rangle
$$

see appendix C2. This is illustrated in Fig. 6. Another kind of transformation is possible for closed ribbons. In particular, we can consider deformations plus inversions of the orientation of the ribbon, as shown in Fig. 6, When $\sigma^{\prime}$ is a transformation of $\sigma$ which includes an inversion we have

$$
K_{\sigma}^{\bar{R}^{C} \bar{C}}|\psi\rangle=K_{\sigma^{\prime}}^{R C}|\psi\rangle .
$$

where $\bar{C}$ is the inverse conjugacy class of $C, \bar{R}^{C}$ is the conjugate representation of $R^{C}$ and $R^{C}$ is an irreducible representation of $\mathbf{N}_{\bar{C}}$ defined by $R^{C}(\cdot):=R(g \cdot \bar{g})$ if $\bar{r}_{C}=$ $\operatorname{gr}_{\bar{C}} \bar{g}$ for some $g \in G$. In the next section we relate this to inversion of topological charge.

\section{Topological charges}

Let $s_{0}, s_{1}$ be two non-adjacent sites in a lattice embedded in the sphere. From the discussion on ribbon operators it follows that the states

$$
|R C ; \mathbf{u v}\rangle:=F_{\rho}^{R C ; \mathbf{u v}}\left|\psi_{G}\right\rangle
$$

form a basis for the subspace with excitations only at $s_{0}$ and $s_{1}$. Here $\left|\psi_{G}\right\rangle$ is the ground state (8) and $\rho$ is any ribbon with $\partial_{i} \rho=s_{i}$.

For each site $s=(v, f)$, we introduce the algebra $\mathcal{D}_{s}$ with basis $\left\{D_{s}^{h g}:=A_{v}^{h} B_{s}^{g}\right\}_{h, g \in G}$. The reason to introduce it is that its action on an excitation at $s$ gives all possible local action on the excitation [18]. In other words,

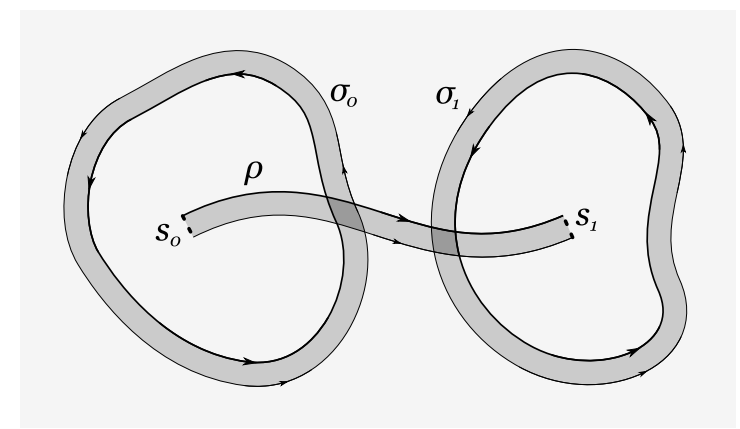

FIG. 7: An open ribbon $\rho$ that connects two sites $s_{0}$ and $s_{1}$ and two closed ribbons $\sigma_{0}$ and $\sigma_{1}$ that surround counterclockwise $s_{0}$ and $s_{1}$, respectively. The ribbon operators $F_{\rho}^{h, g}$ of the open ribbon change the excitations at $s_{0}, s_{1}$. The ribbon operators $K_{\sigma_{0}}^{R C}, K_{\sigma_{1}}^{R C}$ of the closed ribbons project the system onto states with a given topological charge at $s_{0}, s_{1}$.

$\mathcal{D}_{s}$ is useful to show why $\mathbf{u}, \mathbf{v}$ are just local degrees of freedom. The action of the algebras $\mathcal{D}_{s_{i}}$ on the states (21) is

$$
\begin{aligned}
& D_{s_{0}}^{h, g}|R C ; \mathbf{u v}\rangle=\delta_{g, c_{i}} \sum_{s=1}^{n_{R}} \Gamma_{R}^{s j}\left(n\left(h q_{i}\right)\right)|R C ; \mathbf{u}(s) \mathbf{v}\rangle, \\
& D_{s_{1}}^{h, g}|R C ; \mathbf{u v}\rangle=\delta_{g, \bar{c}_{i^{\prime}}} \sum_{s=1}^{n_{R}} \bar{\Gamma}_{R}^{s j^{\prime}}\left(n\left(h q_{i^{\prime}}\right)\right)|R C ; \mathbf{u v}(s)\rangle,
\end{aligned}
$$

where $\mathbf{u}=(i, j), \mathbf{v}=\left(i^{\prime}, j^{\prime}\right), \mathbf{u}(s)=\left(i\left(h q_{i}\right), s\right), \mathbf{v}(s)=$ $\left(i\left(h q_{i^{\prime}}\right), s\right)$ and we set for any $g \in G g=: q_{i(g)} n(g)$ with $n(g) \in \mathbf{N}_{C}$. Equations (22) are a consequence of (B69. 6).

As shown in detail in appendix D it is possible to find operators $d_{\mathbf{u}^{\prime \prime}}^{\mathbf{u}^{\prime}} \in \mathcal{D}_{s_{0}}$ and $d_{\mathbf{v}^{\prime \prime}}^{\mathbf{v}^{\prime}} \in \mathcal{D}_{s_{1}}$ with

$$
d_{\mathbf{u}^{\prime \prime}}^{\mathbf{u}^{\prime}} d_{\mathbf{v}^{\prime \prime}}^{\mathbf{v}^{\prime}}|R C ; \mathbf{u v}\rangle=\delta_{\mathbf{u}, \mathbf{u}^{\prime \prime}} \delta_{\mathbf{v}, \mathbf{v}^{\prime \prime}}\left|R C ; \mathbf{u}^{\prime} \mathbf{v}^{\prime}\right\rangle
$$

Thus we see that a state with particular labels $\mathbf{u}, \mathbf{v}$ can be transformed with local operators into one with any other labels $\mathbf{u}^{\prime}, \mathbf{v}^{\prime}$. Roughly speaking, for local operators we mean operators which act on a neighborhood of the excitations. More exactly, local operators should have a support which does not connect excitations.

What about the degrees of freedom related to the labels $R$ and $C$ ? They can certainly be measured locally, because there exists a set of projectors $D_{s_{0}}^{R C} \in \mathcal{D}_{s_{0}}$ with

$$
D_{s_{0}}^{R C}\left|R^{\prime} C^{\prime} ; \mathbf{u v}\right\rangle=\delta_{R, R^{\prime}} \delta_{C, C^{\prime}}|R C ; \mathbf{u v}\rangle .
$$

However, $R$ and $C$ cannot be changed locally, in the sense that an operator with a support not connecting both sites and which creates no additional excitations will not change their values. To see this, consider two closed ribbons $\sigma_{0}$ and $\sigma_{1}$ that enclose respectively the sites $s_{0}$ and $s_{1}$ counterclockwise, as in Fig. 7. From the discussion in appendix $\mathrm{C} 3$ it follows that

$K_{\sigma_{0}}^{R C}\left|R^{\prime} C^{\prime} ; \mathbf{u v}\right\rangle=K_{\sigma_{1}}^{\bar{R}^{C} \bar{C}}\left|R^{\prime} C^{\prime} ; \mathbf{u v}\right\rangle=\delta_{R, R^{\prime}} \delta_{C, C^{\prime}}|R C ; \mathbf{u v}\rangle$. 


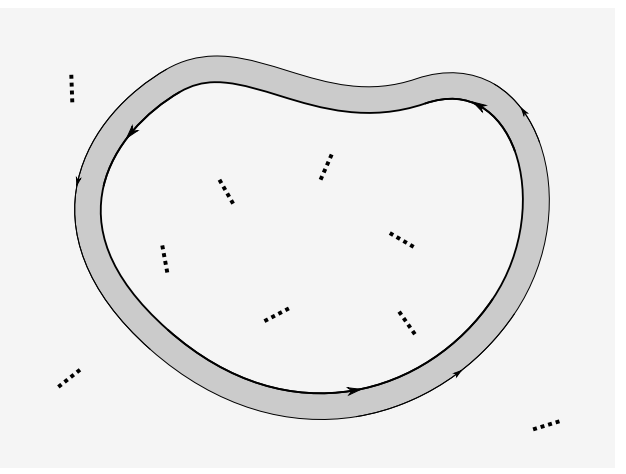

FIG. 8: A boundary ribbon $\sigma$ that encloses several excitations counterclockwise. The corresponding operators $K_{\sigma}^{R C}$ are projectors onto the sector with total topological charge $(R, C)$ inside the ribbon.

Any operator with no common support with $\sigma$ will commute with the projectors $K_{\sigma}^{R C}$, and thus cannot change the value of $R$ and $C$. In particular, any operator which changes $R$ and $C$ must have a support that connects the sites $s_{0}$ and $s_{1}$.

Indeed, the preceding discussion shows that $R$ and $C$ are the labels of the topological charges of the model. Thus the charge of an excitation is the pair $(R, C)$, with $C$ a conjugacy class of $G$ and $R$ an irreducible representation of $\mathbf{N}_{C}$. If a closed ribbon $\sigma$ encloses certain amount of excitations, as in Fig. 8, the projectors $K_{\sigma}^{R C}$ correspond to sectors with different total topological charge in the region surrounded. If $|\xi\rangle$ is a state with no excitations in the area enclosed by $\sigma$, we have

$$
K_{\sigma}^{e 1}|\xi\rangle=|\xi\rangle,
$$

with $e$ the identity representation, see appendix $\mathbf{F}$ Thus, $(e, 1)$ is the trivial charge. This offers a way to describe the ground state of (11) as the space of states for which (26) holds for any boundary ribbon, that is, any closed ribbon enclosing a disc or simply connected region.

In a region with no excitations, quasiparticles can only be locally created in pairs, so that the two excitations have opposite charges and the total charge in the region remains trivial. From (20) or (25) it follows that the opposite of the charge $(R, C)$ is $\left(\bar{R}^{C}, \bar{C}\right)$,

\section{E. Single-quasiparticle states}

In a sphere there do not exist states with a single excitation. The reason, as shown in Fig. 9, is that any closed ribbon $\sigma$ divides the sphere in two regions, both of them simply connected. The ribbon $\sigma$ surrounds one of this region counterclockwise, call it $R_{1}$, and the other one clockwise, call it $R_{2}$. Then the operator $K_{\sigma}^{R C}$ is a projector onto the subspace with total charge $(R, C)$ in $R_{1}$, but also a projector onto the subspace with total charge $\left(\bar{R}^{C}, \bar{C}\right)$ in $R_{2}$. Thus, if there are no excitations

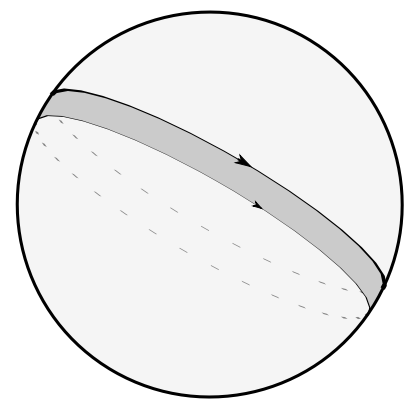

FIG. 9: A closed ribbon in a sphere. Its ribbon operators $K_{\sigma}^{R C}$ project onto states with topological charge $(R, C)$ in the upper side of the sphere and $\left(\bar{R}^{C}, \bar{C}\right)$ in the lower side.

in $R_{1}$, we have a total charge $(e, 1)$ in $R_{1}$ and also a total charge $(e, 1)$ in $R_{2}$. But a single excited site cannot have trivial charge, and thus $R_{2}$ contains either zero or more than one excitation

What about surfaces with non-trivial topology, such as a torus? In the case of Abelian groups, the situation is the same as in the sphere: there are no states with a single excitation. In the case of vertex excitations, that is, electric charges, this follows from the fact that

$$
\prod_{v \in V} A_{v}^{g}=1
$$

For face excitations, that is, magnetic charges, an analogous result holds. For any character $\chi$ of $G$, let $B_{f}^{\chi}:=$ $\sum_{g \in G} \chi(g) B_{s}^{g}$ for $s=(v, f)$. Then

$$
\prod_{f \in F} B_{f}^{\chi}=1 .
$$

For non-Abelian groups, the situation is very different. In fact, examples of single-quasiparticle states can be constructed, see appendix (E).

\section{CONDENSATION AND CONFINEMENT}

\section{A. The models}

We want to modify the Hamiltonian $H_{G}$ by introducing single qudit terms. In particular, we propose to consider projectors of the form

$$
L_{\tau}^{N}:=\frac{1}{|N|} \sum_{n \in N} L_{\tau}^{n}, \quad T_{\tau}^{M}:=\sum_{m \in M} T_{\tau}^{m},
$$

where $\tau$ is a dual or direct triangle and $N, M$ are subgroups of $G$. Thus $L_{\tau}^{N}$ projects out the trivial representation of $N$ and $T_{\tau}^{M}$ selects those states within $M$. We want to have single qudit operators that do not depend on the orientation of the edge $e=e_{\tau}$. This is automatic for $T_{e}^{M}:=T_{\tau}^{M}$, but in the case of dual triangles this is true if and only if $N$ is normal, so that we can set 
$L_{e}^{N}:=L_{\tau}^{N}$. That is, if $n \in N$ and $g \in G$, then $g n \bar{g} \in N$. Moreover, we want these two kinds of single-qudit terms to commute

$$
\left[L_{e}^{N}, T_{e}^{M}\right]=0,
$$

which is true if and only if $N \subset M$.

Now consider a Hamiltonian of the form

$$
H=H_{G}-\mu \sum_{e}\left(L_{e}^{N}+T_{e}^{M}\right)
$$

where $\mu$ is a positive coupling constant and the sum runs over edges $e$. The problem with this Hamiltonian is that the new terms do not commute with $H_{G}$. However, as we show now, we can still consider the limit of large $\mu$. In this limit, the low energy sector is projected out by

$$
P:=\bigotimes_{e} T_{e}^{M} L_{e}^{N}
$$

Let us define the following vertex and face projectors

$$
A_{v}^{M}:=\frac{1}{|M|} \sum_{m \in M} A_{v}^{m}, \quad B_{f}^{N}:=B_{s}^{N}:=\sum_{n \in N} B_{s}^{n},
$$

where $s=(v, f)$ is a site. Note that $B_{s}^{N}$ only depends on $f$ because $N$ is normal. We now make the following observation

$$
\begin{aligned}
|M| P A_{v}^{M} P & =|G| P A_{v} P, \\
P B_{f}^{N} P & =|N| P B_{f} P .
\end{aligned}
$$

Thus, studying the low energy sector of (31) for large $\mu$ amounts to study the sector with no edge excitations of the Hamiltonian

$$
H_{G}^{N, M}:=-\sum_{v} A_{v}^{M}-\sum_{f} B_{f}^{N}-\sum_{e}\left(T_{e}^{M}+L_{e}^{N}\right) .
$$

The point of these Hamiltonians is that all its vertex, face and edge terms commute and thus the ground state of the system can be exactly given. It turns out that it is related to that of (10) but for the group $G^{\prime}:=M / N$, as we will see in the next section. Note that $H_{G}^{1, G}$ is just the original Hamiltonian (11), up to a constant. Although we have motivated the introduction of (35) through (31), our aim is to study the models $H_{G}^{N M}$ in their own right, for arbitrary subgroups $N \subset M \subset G$ with $N$ normal.

\section{B. Ground state}

The ground state of Hamiltonian (35) is described by the conditions

$$
A_{v}^{M}|\psi\rangle=B_{f}^{N}|\psi\rangle=L_{e}^{N}|\psi\rangle=T_{e}^{M}|\psi\rangle=|\psi\rangle
$$

where $v$ is any vertex, $f$ any face and $e$ any edge. Violations of these conditions amount to vertex, face or edge excitations. Let $V$ be the subspace of states with no edge excitations, which is projected out by the projector $P$ of (32). $V$ is a tensor product of single qudit subspaces $V:=\bigotimes_{e} V_{M / N}$, with $V_{M / N} \subset \mathcal{H}_{G}^{\prime}$ the subspace with orthonormal basis:

$$
|\tilde{m}\rangle:=|N|^{-\frac{1}{2}} \sum_{n \in N}|m n\rangle, \quad \tilde{m} \in M / N .
$$

Thus $V \simeq \mathcal{H}_{M / N}$, that is, within the subspace $V$ we are effectively dealing with qudits of dimension $|M / N|$ which are naturally labeled through the group quotient. We denote the corresponding isomorphism by

$$
p: \mathcal{H}_{M / N} \longrightarrow V \text {. }
$$

Let us write

$$
H_{M / N}^{\prime}:=p H_{M / N} p^{-1},
$$

that is, $H_{M / N}^{\prime}$ is the Hamiltonian (11), for the group $M / N$, applied to the subspace $V_{M / N}$. We have

$$
H_{M / N}^{\prime} P=\left(H_{G}^{N, M}+2|E|\right) P .
$$

Thus, within the sector with no edge excitations we are effectively dealing with the Hamiltonian $H_{M / N}$ (1). Moreover, the ground state of $H_{G}^{N, M}$ in $\mathcal{H}_{G}$ is that of $H_{M / N}^{\prime}$ in $V$. The projector onto the ground state is

$$
P_{\mathrm{GS}}^{N, M}:=P \prod_{v} A_{v}^{M} \prod_{f} B_{f}^{N}=P \prod_{v} A_{v}^{\prime} \prod_{f} B_{f}^{\prime}
$$

where $A_{v}^{\prime}:=p A_{v} p^{-1}, B_{f}^{\prime}:=p B_{f} p^{-1}$ with $A_{v}$ and $B_{f}$ acting in $\mathcal{H}_{M / N}$. In the sphere, the normalized ground state is

$$
\left|\psi_{G}^{N, M}\right\rangle \propto P_{\mathrm{GS}}^{N, M}|\mathbf{1}\rangle \propto \prod_{v} A_{v}^{\prime}|\tilde{\mathbf{1}}\rangle .
$$

Thus the new edge terms in the Hamiltonian, which can be thought of as a sort of generalized 'Zeeman terms' [60], have the role of selecting a particular sector of the Hilbert space in which a new non-Abelian discrete gauge symmetry appears, namely $G^{\prime}=M / N$. Thus, edge terms amount to an explicit symmetry breaking mechanism, since in general the gauge symmetry is reduced, even to a trivial one if $M=N$. Alternatively, we can say that they provide a symmetry-reduction mechanism. Thus, the sector with no edge excitations is completely understood. In the remaining sections we study the meaning of edge excitations.

\section{An example}

Before we go on with the general case and its details, let us first give a flavor of what is going on by considering a family of examples. We take $N=1$ and $M$ normal in 
$G$, so that the new gauge group is $G^{\prime}=M$. Note that in this case we can forget about the $L_{e}^{N}$ terms because $L_{e}^{1}=1$. Our aim is to study the result of applying quasiparticle creation operators (16) on a ground state of the Hamiltonian (35):

$$
|\psi\rangle:=F_{\rho}^{R C}(\boldsymbol{\alpha})\left|\psi_{G}^{N M}\right\rangle .
$$

We first consider purely magnetic quasiparticle creation operators, fixing $R$ as the identity representation. For simplicity we set $\alpha^{\mathbf{u v}}=c \in \mathbf{C}$. Then from B69 it follows that $\left[A_{v}^{M}, F^{R C}(\boldsymbol{\alpha})\right]=0$ for every vertex $v$ and from (B50) it follows that $\left[T_{e}^{M}, F^{R C}(\boldsymbol{\alpha})\right]=0$ for any edge $e$ not in a dual triangle of $\rho$. Then due to (36) the state $|\psi\rangle$ can have, at most, face excitations on the ends of $\rho$ and edge excitations on dual triangles of $\rho$. In particular, from (B69, B52 36) it follows that if $f$ is an end face of $\rho$ and $e$ is any dual edge of $\rho$ we have

$$
B_{f}^{1}|\psi\rangle=u|\psi\rangle, \quad T_{e}^{M}|\psi\rangle=u^{\prime}|\psi\rangle,
$$

with $u, u^{\prime}=0,1$. As long as $C \neq 1$, we have $u=0$ and thus $|\psi\rangle$ contains a pair of face excitations. On the other hand, $u^{\prime}=1$ iff $C \subset M$, which means that $|\psi\rangle$ contains a chain of edge excitations along $\rho$ if we try to create magnetic charges which do not belong to the new gauge group $G^{\prime}$. Therefore, we find out that some face excitations are confined, in particular those created with $C \not \subset M$. By this, we mean that the energy of $|\psi\rangle$ increases linearly with the length of $\rho$ in terms of dual triangles.

Next, we consider purely electric quasiparticle creation operators, that is, we set $C=1$. Reasoning in the same way as in the previous case, one finds out that the state $|\psi\rangle$ can have, at most, vertex excitations on the ends of $\rho$, but no face or edge excitations. In particular, if $v$ is an end vertex of $\rho$ we have

$$
A_{v}^{M}|\psi\rangle=u|\psi\rangle
$$

with $u=1$ if the restriction of $R$ to $M$ is an identity representation and $u=0$ otherwise. That is, in some cases $|\psi\rangle$ is a ground state although $R$ is not trivial. Since there is no local degeneracy in the ground state we know that $|\psi\rangle=c\left|\psi_{G}^{N M}\right\rangle$ for some $c \in \mathbf{C}$. Moreover, $c$ can be nonzero, because, as we will see below, for $\alpha^{\mathbf{u v}}=\delta_{\mathbf{u}, \mathbf{v}}$ and $R$ trivial in $M$

$$
\left\langle F_{\rho}^{R 1}(\boldsymbol{\alpha})\right\rangle_{\psi_{G}^{N M}}=1
$$

Moreover, if $\sigma$ is any boundary ribbon we have for $R$ trivial in $M$

$$
\left\langle K_{\sigma}^{R 1}\right\rangle_{\psi_{G}^{N M}}=\frac{n_{R}|M|}{|G|} .
$$

Thus, those electric charges with trivial restriction of $R$ to $M$ are condensed: they are part of the ground state.

\section{Condensation}

Let $\sigma$ be a boundary ribbon, that is, a ribbon that encloses some region $r$. Motivated by the previous example, we want to study the expectation value in the ground state of $H_{G}^{N M}$ of the operators $K_{\sigma}^{R C}$. Recall that these operators project onto the space with total topological charge $(R, C)$ in systems with Hamiltonian $H_{G}$. Then if for a particular charge type we have

$$
\left\langle K_{\sigma}^{R C}\right\rangle:=\left\langle\psi_{G}^{N, M}\left|K_{\sigma}^{R C}\right| \psi_{G}^{N, M}\right\rangle>0
$$

we say that the charges $(R, C)$ of the original Hamiltonian $H_{G}$ get condensed in the system with Hamiltonian $H_{G}^{N M}$ : if one measures the charge of the region $r$ in the ground state of $H_{G}^{N M}$ there exists some probability of finding the charge $(R, C)$.

As we show in appendix $\mathrm{F}$

$$
\left\langle K_{\sigma}^{R C}\right\rangle=\frac{n_{R}|M|}{|G||N|}\left(\chi_{R}, \chi_{e_{M} \uparrow}\right)_{\mathbf{N}_{C}}|C \cap N|,
$$

where the product $(\cdot, \cdot)_{M}$ is defined in A1 and $e_{M} \uparrow$ is the induced representation in $G$ of the identity representation of $M$, see appendix A2 Another way to write the product is

$$
\left(\chi_{R}, \chi_{e_{M} \uparrow}\right)_{\mathbf{N}_{C}}=\frac{1}{\left|\mathbf{N}_{C}\right||M|} \sum_{g \in G}\left|M_{C}^{g}\right|\left(\chi_{R}, 1\right)_{M_{C}^{g}},
$$

where $M_{C}^{g}:=\mathbf{N}_{C} \cap \bar{g} M g$. Note in particular that for $M$ normal the sum has a single term, simplifying the form of (49). The result (49) not only shows that some of the charges are condensed, but also that the expectation value is independent of the shape or size of the ribbon, a feature that underlines the topological nature of the condensation. Such behavior for a perimeter expectation is called a zero law 61 .

Let us consider several examples. First, under Kitaev's original Hamiltonian $H_{G}^{1, G}$ we have

$$
\left\langle K_{\sigma}^{R C}\right\rangle=\delta_{C, 1}, \delta_{R, e_{G}}
$$

where $e_{G}$ is the identity representation of $G$. Thus none of the nontrivial charges is condensed, as expected. In the case $N=M=G$ we have

$$
\left\langle K_{\sigma}^{R C}\right\rangle=\frac{|C|}{|G|} \delta_{R, e_{\mathbf{N}_{C}}},
$$

which means that the purely magnetic charges are condensed. On the contrary, in the case $N=M=1$ we have

$$
\left\langle K_{\sigma}^{R C}\right\rangle=\frac{n_{R}^{2}}{|G|} \delta_{C, 1}
$$

which means that the purely electric charges are condensed. Another illustrative case is that of an Abelian group $G$. In that case we can label the projectors as $K_{\sigma}^{\chi, g}$ 
with $g \in G$ and $\chi$ is an element of the character group of $G$. Then

$$
\left\langle K_{\sigma}^{\chi, g}\right\rangle=\frac{|M|}{|G||N|} \delta_{g N, N} \delta_{\chi_{M}, e_{M}}
$$

where $\chi_{M}$ is the restriction of $\chi$ to $M$.

It is possible to show which charges condense using another kind of expectation values, namely those for operators (16), which create a particle-antiparticle pair in the original model (1). From the discussion in appendix F it follows that if $|C \cap N|=\emptyset$ or $\left(\chi_{R}, 1\right)_{M_{C}^{g}}=0$ for all $g$, we have

$$
\left\langle F_{\rho}^{R C}(\boldsymbol{\alpha})\right\rangle=0
$$

In the case of $M$ normal, for a charge $(R, C)$ that is not condensed according to (49) the operator $F_{\rho}^{R C}(\boldsymbol{\alpha})$ always has expectation value zero. Even if $M$ is not normal, if we trace out the local degrees of freedom and set $F_{\rho}^{R C}:=$ $F_{\rho}^{R C}(\boldsymbol{\alpha})$ with $\alpha^{\mathbf{u v}}=\delta_{\mathbf{u}, \mathbf{v}}$ we get

$$
\left\langle F_{\rho}^{R C}\right\rangle=\frac{1}{|C|}\left(\chi_{R}, \chi_{e_{M} \uparrow}\right)_{\mathbf{N}_{C}}|C \cap N| .
$$

Therefore, for that particular choice we get an expectation value which vanishes if an only if (49) does, showing that both approaches agree. Again topology makes its appearance in the fact that the length or shape of the ribbon $\rho$ are not relevant. For Abelian groups (56) reads

$$
\left\langle F_{\rho}^{\chi, g}\right\rangle=\delta_{g N, N} \delta_{\chi_{M}, e_{M}}
$$

\section{E. Confinement}

The example studied in (IIIC) suggests that the edge terms $L_{e}^{N}$ and $T_{e}^{M}$ could be interpreted as string tension terms, which in turn would confine some of the charges of the original model $H_{G}$. However, one has to be a bit cautious with such a viewpoint in general. Certainly, in those cases in which $N$ is central in $M$ and $M$ is normal (from now on, case I) such a viewpoint makes sense. Only in those cases do certain properties hold, see appendix B 6. In case I we can write the relations, see (B52),

$$
P_{e, e^{\prime}}^{N M} F_{\rho}^{R C ; \mathbf{u v}} P_{e, e^{\prime}}^{N M}=d_{R C}^{N M} F_{\rho}^{R C ; \mathbf{u v}} P_{e, e^{\prime}}^{N M}
$$

where $d_{R C}^{N M}$ equals one (zero) if $C \subset M$ and the restriction of $R$ to $N \subset N_{C}$ is trivial (in other case), $P_{e, e^{\prime}}^{N M}=L_{e}^{N} T_{e^{\prime}}^{M}$ and $e=e_{\tau}, e^{\prime}=e_{\tau^{\prime}}$ with $\tau, \tau^{\prime}$ direct and dual triangles in an open ribbon $\rho$, respectively. The relation (58) show that edge operators project out certain states among those which were created by applying a string operator to a ground state. Note that the projection only takes into account the quasiparticle labels $R$, $C$ of the string operators. Moreover, it is not important which the particular edges are. Outside of case I such nice properties, reasonable for string tension terms, do not hold. As a consequence of (58), we now that a state of the form (43) will have a chain of edge excitations along $\rho$ unless $C \subset M$ and $R$ is trivial in $N$ : all other charges get confined when moving from $H_{G}$ to $H_{G}^{N M}$, which means that they exist at the end of chains of edge excitations. We shall refer to these chains of excitations as domain walls. They can be labeled just as we labeled topological charges in $H_{G}$, something that we will do in the next section.

Unfortunately, as soon as any of the mentioned conditions for case I fails many nice properties of the models are lost. Indeed, only for those systems that fall in that class will we be able to classify domain walls and confined charges in terms of open an closed ribbon operator algebras, in the fashion of what we already did for topological charges in $H_{G}$. On the other hand, in certain more general cases it is still possible to classify domain wall types. In particular, we will show that this can be done in all models with $N$ abelian (from now on, the case II). Interestingly enough, the domain wall fluxes in case II are qualitatively richer than in case I. Such fluxes have in general a non-abelian character for case II systems, whereas for case I they have always an abelian nature.

\section{F. Case I systems}

This section is devoted to those models $H_{G}^{N, M}$ with $N$ central in $M$ and $M$ normal in $G$. We will show that edge excitations appear in the form of domain walls that terminate in certain site excitations which are therefore confined. With this goal in mind, we start introducing the excitations which will turn out to be confined. Consider the projectors

$$
A_{v}^{N}:=\frac{1}{|N|} \sum_{n \in N} A_{v}^{n}, \quad B_{f}^{M}:=B_{s}^{M}=\sum_{m \in M} B_{s}^{m} .
$$

They commute among each other and with the terms of Hamiltonian (35), so that we could choose the energy eigenstates to be eigenstates of the projectors (59). We say that the state $|\psi\rangle$ has a confined excitation at a site $s=(v, f)$ whenever $A_{v}^{N} B_{f}^{M}|\psi\rangle=0$. That these are really excitations follows from

$$
A_{v}^{N} B_{f}^{M}|\psi\rangle=0 \quad \Longrightarrow \quad A_{v}^{M} B_{f}^{N}|\psi\rangle=0
$$

where we have used $A_{v}^{N} A_{v}^{M}=A_{v}^{M}$ and $B_{f}^{M} B_{f}^{N}=B_{f}^{N}$. That they are really confined will be revealed later, but we can already give a clue: a state with a confined excitation at the site $s$ must have an edge excitation at least at one of the edges $e$ meeting at $v$ or in the border of $f$. Thus, confined excitations cannot appear isolated: there must be edge excitations around them. Conversely, it is also true that a chain of edge excitations cannot terminate without a confined excitation in its end. However, this is not enough to demonstrate confinement, but we can do it better by introducing suitable ribbon operator algebras. 


\section{Open ribbon operators.}

Domain walls have a type, and this type behaves as a flux in the absence of confined excitations. We thus need a family of projectors that distinguish between the domain wall fluxes that cross a particular line, analogous to the projectors $K_{\sigma}^{R C}$ that distinguished the topological charge in a the area enclosed by $\sigma$. So we consider as our starting point the ribbon operator algebra $\mathcal{F}_{\rho}$ for an open ribbon $\rho$. Since the flux should be the same if we move the ends of our flux-measuring ribbon in an area with no edge excitations, we need a ribbon algebra which to some extent forgets the ends of the ribbon. In particular, we should choose ribbon operators that do not create or destroy excitations. Thus, we define $\mathcal{J}_{\rho} \subset \mathcal{F}_{\rho}$ as the subalgebra containing those operators $F \in \mathcal{F}_{\rho}$ which commute with all vertex operators $A_{v}^{M}$ and face operators $B_{f}^{N}$. Such operators also commute with all edge terms $L_{e}^{N}$ and $T_{e}^{M}$, see colollary 12 .

As we show in proposition 11, $\mathcal{J}_{\rho}$ is linearly generated by certain orthogonal projectors $J_{\rho}^{\chi t}$ that form a resolution of the identity. The labels $(\chi, t)$ of these projectors are $\chi$, an element of the character group of $N$, and $t$, an element of the quotient group $G / M$. If $|\psi\rangle$ has no edge excitations along $\rho$ then

$$
J_{\rho}^{e 1}|\psi\rangle=|\psi\rangle,
$$

where $e$ and 1 are both identity elements, see (F4 B85).

The algebra $\mathcal{J}_{\rho}$ can stand deformations in which the ends of $\rho$ are not fixed. More exactly, if the state $|\psi\rangle$ is such that the open ribbon $\rho$ can be deformed, without fixing its ends, to obtain another ribbon $\rho^{\prime}$ in such a way that no confined excitations are crossed and the ends do not touch edge excitations, then

$$
J_{\rho}^{\chi t}|\psi\rangle=J_{\rho^{\prime}}^{\chi t}|\psi\rangle,
$$

see appendix C2. This is illustrated in Fig. 10. As in the case of closed ribbons in $H_{G}$, here we can consider inversions in the orientation of the ribbon, as shown in Fig. 10, When $\rho^{\prime}$ is a transformation of $\rho$ which includes an inversion we have

$$
J_{\rho}^{\chi t}|\psi\rangle=J_{\rho^{\prime}}^{\bar{\chi}^{t} \bar{t}}|\psi\rangle
$$

where for any $n \in N$ we set $\chi^{t}(n):=\chi(\operatorname{tn} \bar{t})$, see appendix C2 2

When a ribbon $\rho_{2}$ crosses two domain walls which are respectively crossed by two other ribbons $\rho_{3}, \rho_{4}$ with the same orientation as $\rho$, as in Fig. 12, we have

$$
J_{\rho_{2}}^{\chi t}=\sum_{\xi} \sum_{k \in G / M} J_{\rho_{3}}^{\bar{\xi}^{k}} J_{\rho_{4}}^{\xi \chi k t}
$$

where $\xi$ runs over the group of characters, see appendix B10.

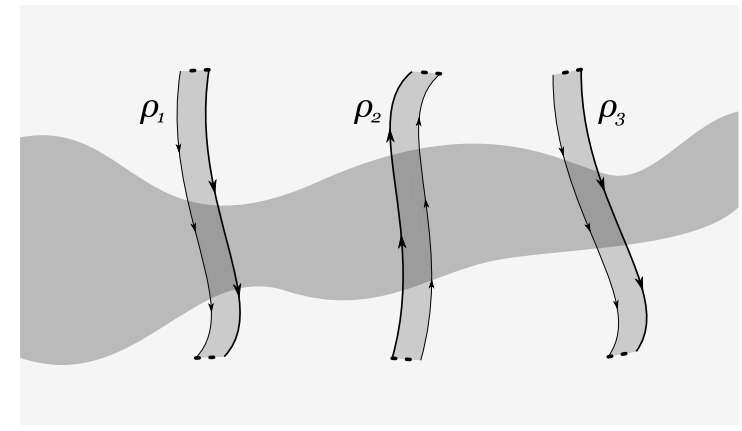

FIG. 10: Examples of open ribbon transformations for $\mathcal{J}_{\rho}$. Edge excitations are present only in the shaded area. No confined quasiparticle excitations are present. The ribbon $\rho_{1}$ is a deformation of $\rho_{3}$, and $\rho_{2}$ has an inverse orientation with respect to them.

\section{Closed ribbon operators.}

Before we can further analyze the consequences of the properties of $\mathcal{J}_{\rho}$, as we shall do in the next section, we have to introduce a family of projectors that distinguish the charges in our models. The situation is different to the one we found in the models $H_{G}$, because now some charges are confined, and thus the rules for deforming closed ribbons will change qualitatively to reflect this fact. Our starting point is the ribbon operator algebra $\mathcal{F}_{\sigma}$ for a closed ribbon $\sigma$, from which we want to select certain suitable operators, just as we did for other projector algebras. In the case of open ribbons just considered, we made this selection requiring that the operators commuted with all vertex and face terms. Here that will not be enough, because since $\sigma$ is closed we would be considering operators that create a closed domain wall, with no ends and no confined excitations. Therefore, we define $\mathcal{K}_{\sigma}^{\prime} \subset \mathcal{F}_{\sigma}$ as the subalgebra containing those operators $K \in \mathcal{F}_{\sigma}$ which commute with all vertex operators $A_{v}^{M}$, face operators $B_{f}^{N}$ and edge operators $L_{e}^{N}, T_{e}^{M}$.

As we show in proposition 14. $\mathcal{K}_{\sigma}^{\prime}$ is linearly generated by certain orthogonal projectors $K_{\sigma}^{R C}$ that form a resolution of the identity. The labels $(R, C)$ of these projectors are $C$, a set of the form $\{m g \bar{m} \mid m \in M\}$ for some $g$ in $G$, and $R$, an irreducible representation of the group $\mathbf{N}_{C}^{\prime}:=\left\{m \in M \mid m r_{C} \bar{m} \bar{r}_{C} \in N\right\}$ for some fixed $r_{C} \in C$. If $\sigma$ is a boundary ribbon surrounding an area with no vertex or face excitations in the state $|\psi\rangle$, then

$$
K_{\sigma}^{e 1}|\psi\rangle=|\psi\rangle
$$

where $e$ and 1 are both identity elements, see F6, B86. B75).

The algebra $\mathcal{K}_{\sigma}^{\prime}$ can stand deformations in which the end $\partial \sigma$ is not fixed, as long as it crosses no domain walls. If the state $|\psi\rangle$ is such that the open ribbon $\sigma$ can be deformed to obtain another ribbon $\sigma^{\prime}$ in such a way that no vertex or face excitations are crossed and the end of 


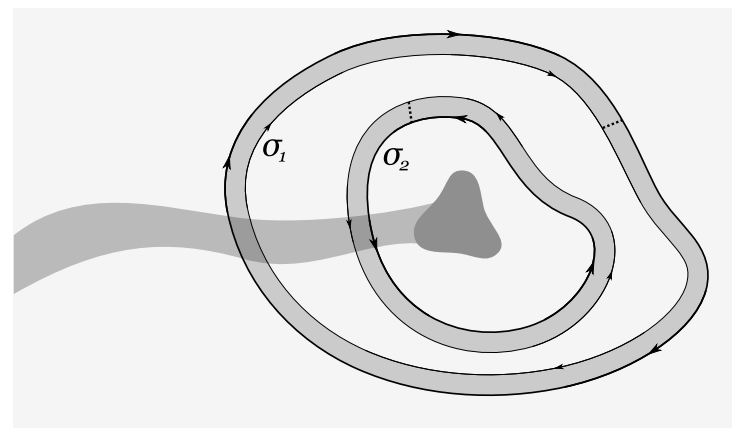

FIG. 11: Examples of closed ribbon transformations for $\mathcal{K}_{\rho}^{\prime}$. The light shaded area represents edge or domain wall excitations, and the dark shaded area confined quasiparticle excitations. The ribbon $\sigma_{1}$ is a deformation of $\sigma_{2}$ which includes an inversion.

$\sigma$ touches no edge excitations, then

$$
K_{\sigma}^{R C}|\psi\rangle=K_{\sigma^{\prime}}^{R C}|\psi\rangle,
$$

see appendix C2 This is illustrated in Fig. 11] As in the case of closed ribbons in $H_{G}$, here we can consider inversions in the orientation of the ribbon, as shown in Fig. 11. When $\sigma^{\prime}$ is a transformation of $\sigma$ which includes an inversion we have

$$
K_{\sigma}^{R C}|\psi\rangle=K_{\sigma^{\prime}}^{\bar{R}^{C} \bar{C}}|\psi\rangle,
$$

where $R^{C}$ is an irreducible representation of $\mathbf{N}_{\bar{C}}^{\prime}$ defined by $R^{C}(\cdot):=R(m \cdot \bar{m})$ if $\bar{r}_{C}=m r_{\bar{C}} \bar{m}$ for some $m \in M$, see appendix $\mathrm{C} 2$.

\section{Domain walls and charges.}

The deformation properties of the projectors in $\mathcal{J}_{\rho}$ that we have introduced indicate that edge excitations appear in the form of domain walls to which a flux can be attached. Branching points are possible in these walls. Each value of the flux corresponds to a projector $J_{\rho}^{\chi t}$, and we know that it is preserved along a domain wall due to the deformation property (62). The trivial flux is given by (61) and the inverse flux by (63). Since deformations of $J_{\rho}^{\chi t}$ only require that no confined excitations are crossed, a domain wall can only end in the presence of confined excitations. Domain wall fluxes have an abelian nature: as indicated by (64), the addition of two given fluxes always produces the same combined flux. All these ideas are reflected in Fig. 12 .

Regarding charge labeling, the properties of the projectors in $\mathcal{K}_{\sigma}^{\prime}$ very much resemble those already found in the study of $\mathcal{K}_{\sigma}$ in systems with Hamiltonian $H_{G}$. The trivial charge is given by (65) and the inverse charge by (67). Indeed, the new element that appears is that now the deformation properties (66) take into account that the charge could be attached to a domain wall.

But if we want to neatly describe confinement, we have to establish the relationship between domain walls and charges. To this end, consider Fig. 12, We can deform each ribbon $\rho_{i}$, without changing the flux it measures, till $\rho_{i}$ is equal to $\sigma_{i}$, the boundary ribbon enclosing the charge at the end of the domain wall. At that point we can compare both projector algebras, with the following result: there exists a function $f$ onto the group character of $N$ such that

$$
J_{\sigma}^{\chi t}=\sum_{C \subset t M} \sum_{R} \delta_{\chi, f(R)} K_{\sigma}^{R C}
$$

where the sum on $R$ runs over irreducible representations in $\mathbf{N}_{C}^{\prime}$, see (B90). Equation (68) tells us at the end of which domain walls can each charge exist. In other words, the projectors in $\mathcal{J}_{\sigma}$ classify charge types from $\mathcal{K}_{\sigma}^{\prime}$ in different compartments or sectors: each of these sectors gives a confined charge type. The different charge labels within a sector give us the topological part of the charge. In particular, for the trivial confined charge we recover the topological charge types for a system with Hamiltonian $H_{M / N}$, in accordance with the study of the ground state of section [IIB, Finally, all charges which do not belong to the trivial confined charge sector are indeed confined, because if we take any circle surrounding them we must always have a domain wall crossing it. When excitations are localized in a single site, it turns out that confined excitations are exactly described, as expected, by the projectors (59).

As we already did in the particular case of $H_{G}$ models, the ground state can be described in terms of ribbon operators of arbitrary size. In this case, we have to impose that no region should contain a nontrivial charge and no line should be crossed by a nontrivial domain wall flux. That is, a state $\xi$ is a ground state if and only if

$$
K_{\sigma}^{e 1}|\xi\rangle=|\xi\rangle, \quad J_{\rho}^{e 1}|\xi\rangle=|\xi\rangle,
$$

for all boundary ribbons $\sigma$ and proper ribbons $\rho$. This conditions generalize to arbitrary $N$ and $M$, see appendix F

\section{G. Case II systems}

This section is devoted to those models $H_{G}^{N, M}$ with $N$ abelian. As we have already commented, in this case we will only be able to describe, using ribbon projector algebras, domain wall fluxes, but not charge types, except those in the sector with no edge excitations which are already classified through the mapping to $H_{M / N}$. Thus we proceed to describe the algebra $\mathcal{J}_{\rho}$, which is defined exactly as in case I. As discussed in appendix B10, in this case the projectors are $J_{\rho}^{R T}$ with $T$ an element of the double coset $M \backslash G / M$ and $R$ an induced representation in $\mathbf{N}_{T}$ of an irreducible representation in $N$, with $\mathbf{N}_{T}$ the group $\left\{m \in M \mid m r_{T} M=r_{T} M\right\}$ for some fixed $r_{T} \in T$. We recall that each element of the double coset takes the form $T=\left\{m r_{T} m^{\prime} \mid m, m^{\prime} \in M\right\}$. From the double coset structure, we can obtain a subalgebra of the group 


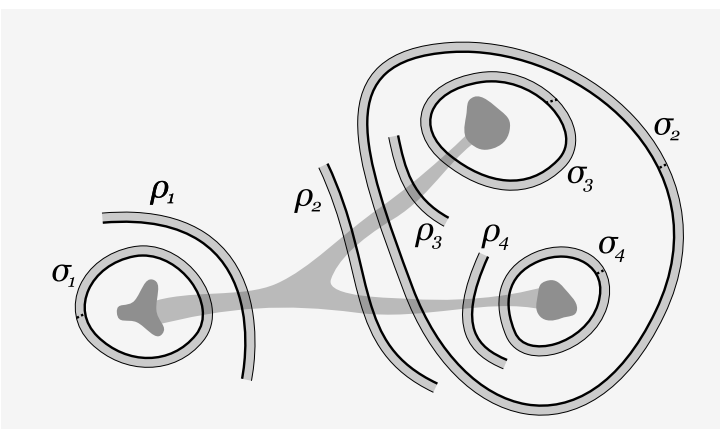

FIG. 12: An illustration of the relationship between domain wall fluxes and quasiparticle charges. The light shaded area represents edge or domain wall excitations, and the dark shaded area quasiparticle excitations. Each of the open ribbons $\rho_{i}$ can be deformed to the boundary ribbon $\sigma_{i}$, so that the domain wall flux measured by $\rho_{i}$ corresponds to the confined charge measured by $\sigma_{i}$. Since $\rho_{1}$ and $\rho_{2}$ are equivalent up to an inversion, the confined charges measured by $\sigma_{1}$ and $\sigma_{2}$ are inverses. The total flux in $\rho_{2}$ is the combination of that in $\rho_{3}$ and $\rho_{4}$, and thus the confined charge measured by $\sigma_{2}$ is the combination of that measured by $\sigma_{3}$ and $\sigma_{4}$.

algebra $\mathbf{C}(G)$. Indeed, for $T, T^{\prime} \in M \backslash G / M$ we have

$$
T T^{\prime}=\sum_{T^{\prime \prime} \in M \backslash G / M} C_{T T^{\prime}}^{T^{\prime \prime}} T^{\prime \prime}
$$

where $C_{T T^{\prime}}^{T^{\prime \prime}}$ are positive integers. The rules for deformations of $J_{\rho}^{R T}$, discussed in appendix C2, state that the ribbon $\rho$ can move across regions in which $B_{f}^{N}|\psi\rangle=|\psi\rangle$ and $A_{v}^{N}|\psi\rangle=|\psi\rangle$ are satisfied, and the ends can be displaced as long as they do not touch edge excitations, with the result that the flux measured by $J_{\rho}^{R T}$ does not change. The inverse flux of $(R, T)$ is $\left(\bar{R}^{T}, \bar{T}\right)$, where $\bar{M}$ contains the inverses of the elements of $M$ and $R^{T}$ is defined by $R^{T}(\cdot):=R\left(r_{T} m \cdot \bar{m} \bar{r}_{T}\right)$ if $m \in M$ is such that $M=r_{T} m r_{\bar{T}} M$. Finally, there is no analog of (64), in the sense that the knowledge of two fluxes is not enough to determine the total combined flux. This can be seen for example in (70). When $M$ is normal (case I), the sum is reduced to a single term, so that combining a flux $(e, T)$ with a flux $\left(e, T^{\prime}\right)$ will give certain determinate total flux $\left(e, T^{\prime \prime}\right)$. This is no longer true when $M$ is not normal (case II), giving a non-abelian nature to the domain wall fluxes, which disappears in case I.

\section{CONCLUSIONS}

In this paper we have introduced a family of quantum lattice Hamiltonians with a discrete non-Abelian gauge symmetry such that the standard Kitaev model for topological quantum computation is a particular case of this class. The ground state of the models can be exactly given and, in many cases, quasiparticles or at least domain wall excitations can be classified. They can be characterized by operator algebras corresponding to closed and open ribbon operators. This is done in full generality for arbitrary topologies. The models can be understood in terms of topological charge condensation and confinement with respect to the standard Kitaev models.

We have given a detailed account of the quasiparticle excitations in the standard non-Abelian Kitaev model. In particular, we have seen that for orientable closed surfaces other than the sphere, like the torus, excitations may show up in the form of single quasiparticles.

One of the features exhibited by the family of nonAbelian models considered in this paper is the existence of a string tension for the motion of quasiparticle excitations. It would be interesting to study the role of such tensions when the action of external source of decoherence such as local external fields or thermal effects are studied [30], 62]. Another aspect that deserves further study is the properties of these models for topological quantum computation, since here we have only focused on their properties as far as topological order is concerned.

Acknowledgements We acknowledge financial support from a PFI grant of the EJ-GV (H.B.), DGS grants under contracts BFM 2003-05316-C02-01, FIS2006-04885 (H.B., M.A.M.D,), and the ESF Science Programme INSTANS 2005-2010 (M.A.M.D.).

\section{APPENDIX A: GROUP ALGEBRAS}

We present some basic properties of the algebras of finite groups. We will find them useful for establishing several results on ribbon operator algebras in the next section. In particular, we need them to label the charges and domain walls of our models.

\section{Representations and classes}

Given a finite group $G$, let $(G)_{\mathrm{cj}}$ be the set of conjugacy classes of $G$ and $(G)_{\text {ir }}$ be the set of irreducible representations of $G$, up to isomorphisms [63]. For $R \in(G)_{\mathrm{ir}}, g \in G$, we denote by $R_{g}$ the image of $g$ under $R$ and by $\Gamma_{R}(g)$ the unitary matrix of $R_{g}$ in a particular basis. The character of a representation $R$ is $\chi_{R}(g):=\sum_{i} \Gamma_{R}^{i i}(g)$. Characters are examples of class functions $\phi, \psi:(G)_{\mathrm{cj}} \longrightarrow \mathbf{C}$, for which we introduce the product 63]

$$
(\phi, \psi)_{G}:=\frac{1}{|G|} \sum_{C \in(G)_{\mathrm{cj}}}|C| \phi(C) \bar{\psi}(C),
$$

where the bar denotes complex conjugation.

The following are the well-known orthogonality relations for irreducible representations, characters and con- 
jugacy classes [63

$$
\begin{aligned}
\sum_{g \in G} \Gamma_{R}^{i j}(g) \bar{\Gamma}_{R^{\prime}}^{i^{\prime} j^{\prime}}(g) & =\frac{|G|}{n_{R}} \delta_{R, R^{\prime}} \delta_{i, i^{\prime}} \delta_{j, j^{\prime}}, \\
\sum_{C \in(G)_{\mathrm{cj}}}|C| \chi_{R}(C) \bar{\chi}_{R^{\prime}}(C) & =|G| \delta_{R, R^{\prime}}, \\
\sum_{R \in(G)_{\mathrm{ir}}} \chi_{R}(C) \bar{\chi}_{R}\left(C^{\prime}\right) & =\frac{|G|}{|C|} \delta_{C, C^{\prime}},
\end{aligned}
$$

where $R, R^{\prime} \in(G)_{\mathrm{ir}}, C, C^{\prime} \in(G)_{\mathrm{cj}}$ and $n_{R}=\chi_{R}(1)$ is the degree of $R$. A3A4 imply that $\left|(G)_{\mathrm{cj}}\right|=\left|(G)_{\mathrm{ir}}\right|$. The identities (A3), which are just a particular case of (A2), can be written more concisely as $\left(\chi_{R}, \chi_{R^{\prime}}\right)_{G}=\delta_{R, R^{\prime}}$.

\section{Induced representations}

Given a finite group $G$ and a normal subgroup $H \subset G$, let $(H, G)_{\mathrm{cj}}$ be the set of conjugacy classes of $G$ contained in $H$ and $(H, G)_{\text {ir }}$ be the set of induced representations in $G$ of irreducible representations in $H$, up to isomorphisms [63]. Recall that for each representation $R$ of $H$ there exists a representation $R_{\text {Ind }}$, called the induced representation of $R$ in $G$, such that for $g \in G$

$$
\chi_{R_{\text {Ind }}}(g):= \begin{cases}\sum_{r \in G / H} \chi_{R}(r g \bar{r}), & g \in H, \\ 0, & g \notin H .\end{cases}
$$

The Frobenius reciprocity formula asserts that for $\phi$ : $(G)_{\mathrm{cj}} \longrightarrow \mathbf{C}$ and $R \in(H)_{\mathrm{ir}}$

$$
\left(\left.\phi\right|_{H}, \chi_{R}\right)_{H}=\left(\phi, \chi_{R_{\text {Ind }}}\right)_{G},
$$

where $\left.\phi\right|_{H}$ is the restriction of $\phi$ to $H$.

Let us introduce an equivalence relation in $(H)_{\mathrm{cj}}$. For $D, D^{\prime} \in(H)_{\mathrm{cj}}$, we set $D \sim D^{\prime}$ if $D^{\prime}=g D \bar{g}$ for some $g \in G / H$. Each $C \in(H, G)_{\mathrm{cj}}$ is related to a unique equivalence class $\tilde{C}$ in the following way

$$
C=\bigcup_{D \in \tilde{C}} D .
$$

Given $S \in(H)_{\text {ir }}$ and $r \in G / H$, define a representation $S^{r} \in(H)_{\text {ir }}$ setting $S_{h}^{r}:=S_{r h \bar{r}}$. We introduce an equivalence relation in $(H)_{\mathrm{ir}}$. For $S, S^{\prime} \in(H)_{\mathrm{ir}}$, we set $S \sim S^{\prime}$ if $S=S^{g}$ for some $g \in G / H$. Each $R \in(H, G)_{\text {ir }}$ is related to a unique equivalence class $\tilde{R}$ in the following way

$$
\chi_{R}(g)= \begin{cases}\frac{|G|}{|H||R|} \sum_{S \in \tilde{R}} \chi_{S}(g), & g \in H, \\ 0, & g \notin H,\end{cases}
$$

and $S \in \tilde{R}$ iff $S_{\text {Ind }}=R$.

As a generalization of $\mathrm{A3}$ A4 we have the following orthogonality relations

$$
\begin{aligned}
\sum_{C \in(H, G)_{\mathrm{cj}}}|C| \chi_{R}(C) \bar{\chi}_{R^{\prime}}(C) & =\frac{|G|^{2}}{|H||\tilde{R}|} \delta_{R, R^{\prime}}, \\
\sum_{R \in(H, G)_{\mathrm{ir}}}|\tilde{R}| \chi_{R}(C) \bar{\chi}_{R}\left(C^{\prime}\right) & =\frac{|G|^{2}}{|H||C|} \delta_{C, C^{\prime}},
\end{aligned}
$$

where $R, R^{\prime} \in(H, G)_{\text {ir }}$ and $C, C^{\prime} \in(H, G)_{\mathrm{cj}}$. A9910 imply that $\left|(H, G)_{\mathrm{cj}}\right|=\left|(H, G)_{\text {ir }}\right|$. Note that (A99) can be rewritten in terms of the product $(\cdot, \cdot)_{G}$ and derived from (A6) as follows:

$$
\left(\chi_{R}, \chi_{R^{\prime}}\right)_{G}=\left(\chi_{S_{0}}, \chi_{R^{\prime}}\right)_{H}=\frac{|G|}{|H||\tilde{R}|} \delta_{R, R^{\prime}},
$$

where $S_{0} \in \tilde{R}$. As for (A10), it follows from A8 A4)

$$
\begin{aligned}
& \sum_{R \in(H, G)_{\mathrm{ir}}}|\tilde{R}| \chi_{R}(C) \bar{\chi}_{R}\left(C^{\prime}\right)= \\
& =\sum_{R \in(H, G)_{\mathrm{ir}}} \frac{|G|^{2}}{|H|^{2}|\tilde{R}|} \sum_{S, S^{\prime} \in \tilde{R}} \chi_{S}\left(D_{0}\right) \bar{\chi}_{S^{\prime}}\left(D_{0}^{\prime}\right)= \\
& =\sum_{g \in G / H} \sum_{R \in(H, G)_{\mathrm{ir}}} \frac{|G|}{|H|} \sum_{S \in \tilde{R}} \chi_{S}\left(D_{0}\right) \bar{\chi}_{S}\left(g D_{0}^{\prime} \bar{g}\right)= \\
& \quad=\frac{|G|}{\left|D_{0}\right|} \sum_{g \in G / H} \delta_{D_{0}, g D_{0}^{\prime} \bar{g}}=\frac{|G|^{2}}{|H||C|} \delta_{C, C^{\prime}}, \quad
\end{aligned}
$$

where $D_{0} \in \tilde{C}, D_{0}^{\prime} \in \tilde{C}^{\prime}$.

\section{The group algebra $\mathbf{C}[G]$}

Given a finite group $G$, the group algebra $\mathbf{C}[G]$ consists of formal sums $\sum_{g \in G} c_{g} g, c_{g} \in \mathbf{C}$. We are interested in certain representation $\mathcal{R}: G \times G \longrightarrow \mathbf{G L}(\mathbf{C}[G])$. Let us denote by $\mathcal{R}_{g_{1}, g_{2}}$ the image of $\left(g_{1}, g_{2}\right) \in G \times G$. Then $\mathcal{R}$ is defined by:

$$
\mathcal{R}_{g_{1}, g_{2}}(g):=g_{1} g \bar{g}_{2}, \quad g \in G,
$$

where $\bar{g}$ denotes the inverse of $g$. It turns out that the following isomorphism holds, as we shall check below,

$$
\mathbf{C}[G] \simeq \sum_{R \in(G)_{\mathrm{ir}}} V_{R} \otimes V_{\bar{R}}
$$

where $V_{R}$ is the representation space of $R$.

With the aim of checking (A14) explicitly, let us consider the following elements of $\mathbf{C}[G]$ :

$$
e_{R}^{i j}:=\frac{n_{R}}{|G|} \sum_{g \in G} \bar{\Gamma}_{R}^{i j}(g) g
$$

where $R$ is a representation of $G$ and $i, j=1, \ldots, n_{R}$. For irreducible $R$, there are $|G|$ such elements, because $\sum_{R \in(G)_{\mathrm{ir}}} n_{R}^{2}=|G|$ due to A14). In fact, they give a new basis for $\mathbf{C}[G]$ :

$$
g=\sum_{R \in(G)_{\mathrm{ir}}} \sum_{i, j=1}^{n_{R}} \Gamma_{R}^{i j}(g) e_{R}^{i j}
$$

which can be checked using (A4). In this basis

$$
\mathcal{R}_{g_{1}, g_{2}} e_{R}^{i j}=\sum_{k, l=1}^{n_{R}} \Gamma_{R}^{k i}\left(g_{1}\right) \bar{\Gamma}_{R}^{l j}\left(g_{2}\right) e_{R}^{k l},
$$


which gives explicitly the isomorphism (A14), as desired. Let us define

$$
\overline{\left(\sum_{g} c_{g} g\right)}:=\sum_{g} \bar{c}_{g} \bar{g}
$$

Then, we have

$$
e_{R}^{i j} e_{R^{\prime}}^{i^{\prime} j^{\prime}}=\delta_{R, R^{\prime}} \delta_{j, i^{\prime}} e_{R}^{i j^{\prime}}, \quad \bar{e}_{R}^{i j}=e_{R}^{j i} .
$$

which follow from $\mathrm{A} 2$.

\section{The algebra $\mathcal{Z}_{G}$}

The center of a group algebra $\mathbf{C}[G]$, denoted $\mathcal{Z}_{G}$, is the subalgebra of elements that commute with all the elements of $\mathbf{C}[G]$. A basis for the center of $\mathbf{C}[G]$ is the following:

$$
e_{C}:=\sum_{g \in C} g \quad C \in(G)_{\mathrm{cj}} .
$$

We have

$$
e_{C} e_{C^{\prime}}=: \sum_{C^{\prime \prime} \in(G)_{\mathrm{cj}}} N_{C, C^{\prime}}^{C^{\prime \prime}} e_{C^{\prime \prime}}, \quad \bar{e}_{C}=e_{\bar{C}},
$$

where $\bar{C}$ denotes the inverse class of $C$ and $N_{C, C^{\prime}}^{C^{\prime \prime}} \geq 0$ are integers.

With the aim of finding an alternative basis for $\mathcal{Z}_{G}$, we define

$$
e_{R}:=\sum_{i} e_{R}^{i i}, \quad R \in(G)_{\mathrm{ir}},
$$

which are a nice set of projectors:

$$
\begin{aligned}
e_{R} e_{R^{\prime}} & =\delta_{R, R^{\prime}} e_{R}, \\
\bar{e}_{R} & =e_{R}, \\
\sum_{R \in(G)_{\mathrm{ir}}} e_{R} & =1,
\end{aligned}
$$

as follows from (A4). They provide us with a new basis for $\mathcal{Z}_{G}$ since

$$
\begin{aligned}
& e_{R}=\frac{n_{R}}{|G|} \sum_{C \in(G)_{\mathrm{cj}}} \bar{\chi}_{R}(C) e_{C}, \\
& e_{C}=\sum_{R \in(G)_{\mathrm{ir}}} \frac{|C|}{n_{R}} \chi_{R}(C) e_{R},
\end{aligned}
$$

as can be checked using (A4).

\section{The algebra $\mathcal{Z}_{H, G}$}

Let $H$ be a normal subgroup of $G$. There is a natural inclusion $\mathbf{C}[H] \subset \mathbf{C}[G]$. We are interested in the intersection of their centers $\mathcal{Z}_{H, G}:=\mathcal{Z}_{H} \cap \mathcal{Z}_{G}$. A basis for the algebra $\mathcal{Z}_{H, G}$ is

$$
e_{C}^{G}:=\sum_{g \in C} g \quad C \in(H, G)_{\mathrm{cj}} .
$$

Its elements can be rewritten in terms of elements of $\mathcal{Z}_{H}$ as follows

$$
e_{C}^{G}=\sum_{D \in \tilde{C}} e_{D}^{H}=\frac{|H||\tilde{C}|}{|G|} \sum_{g \in G / H} g e_{D}^{H} \bar{g},
$$

where we are using the notation of (A7).

We have the aim of finding a basis of projectors for $\mathcal{Z}_{H, G}$, analogous to A23. Let

$$
e_{R}^{\prime}:=\frac{|H||\tilde{R}|}{|G|} e_{R}^{G}=\sum_{S \in \tilde{R}} e_{S}^{H}, \quad R \in(H, G)_{\mathrm{ir}},
$$

so that,

$$
\begin{aligned}
e_{R}^{\prime} e_{R^{\prime}}^{\prime} & =\delta_{R, R^{\prime}} e_{R}^{\prime}, \\
e_{R}^{\prime \dagger} & =e_{R}^{\prime} \\
\sum_{R \in(H, G)_{\mathrm{ir}}} e_{R}^{\prime} & =1
\end{aligned}
$$

showing also that the $e_{R}$ are linearly independent. Indeed, $\left\{e_{R}^{\prime} \mid R \in(H, G)_{\text {ir }}\right\}$ is the desired projector basis for $\mathcal{Z}_{H, G}$, because we have

$$
\begin{aligned}
e_{R}^{\prime} & =\frac{n_{R}|\tilde{R}||H|}{|G|^{2}} \sum_{C \in(H, G)_{\mathrm{cj}}} \bar{\chi}_{R}(C) e_{C}^{G}, \\
e_{C}^{G} & =\sum_{R \in(H, G)_{\mathrm{ir}}} \frac{|C|}{n_{R}} \chi_{R}(C) e_{R}^{\prime},
\end{aligned}
$$

where $R \in(H, G)_{\text {ir }}$ and $C \in(H, G)_{\text {cj }}$. In order to check (A31), insert (A30) and apply A10. Finally, not that if $H$ belongs to the center of $G$ we have $e_{R}^{\prime}=e_{R}^{H}$.

\section{APPENDIX B: RIBBON OPERATORS}

In this appendix we discuss ribbon operator algebras. We will first introduce the geometric aspects of ribbons, then we will attach operators to ribbons, and we will finish describing and characterizing certain projector ribbon subalgebras. These projectors are directly related to the topological charges and domain wall fluxes in the models under study.

\section{Sites, triangles and strips}

Our starting point is a lattice embedded in an orientable two-dimensional manifold. Let $V, E$ and $F$ be the sets of its vertices, edges and faces respectively. The 
edges of the direct and dual lattices must be oriented accordingly, as explained in the main text in sect II and Fig 1. A direct edge $e$ points from the vertex $\partial_{0} e$ to the vertex $\partial_{1} e$, and a dual edge $e^{*}$ points from the dual vertex $\partial_{0} e^{*}$ to the dual vertex $\partial_{1} e^{*}$. The shape of the lattice is arbitrary, but with certain conditions. Namely (i) if $e$ is an edge, then $\partial_{0} e \neq \partial_{1} e$ and (ii) a face with $s$ edges must have $s$ different vertices. The same conditions must hold for the dual lattice.

For each edge $e \in E$, we introduce an inverse edge $\bar{e}$ which is an edge with the direction reversed,i.e., with $\partial_{0} \bar{e}=\partial_{1} e$ and $\partial_{1} \bar{e}=\partial_{0} e$. We let $\overline{\bar{e}}=e$ and denote by $E_{\text {ext }}:=E \cup \bar{E}$ the disjoint union of the original and inverse edges. For dual edges, we set $\overline{\left(e^{*}\right)}=(\bar{e})^{*}$ so that $E_{\text {ext }}^{*}=E^{*} \cup \bar{E}^{*}$.

A (direct) path $p$ is a list $\left(v_{0}, e_{1}, v_{1}, \ldots, e_{n}, v_{n}\right)$ such that $v_{i} \in V, e_{i} \in E_{\text {ext }}, \partial_{0} e_{i}=v_{i-1}$ and $\partial_{1} e_{i}=v_{i}$. A dual path $p^{*}$ is a list $\left(f_{0}^{*}, e_{1}^{*}, f_{1}^{*}, \ldots, f_{r}^{*}\right)$ such that $f_{i} \in F$, $e_{i} \in E_{\text {ext }}, \partial_{0} e_{i}^{*}=f_{i-1}^{*}$ and $\partial_{1} e_{i}^{*}=f_{i}^{*}$.

Sites. A site is a pair $s=(v, f)$ with $f$ a face and $v$ one of its vertex. We visualize sites as dashed lines connecting the vertex $v$ and the dual vertex $f^{*}$, as shown in Fig 13 and use the notation $s=:\left(v_{s}, f_{s}\right)$.

Triangles. A direct triangle $\tau=\left(s_{0}, s_{1}, e\right)$ consists of two sites $s_{i}$ and a direct edge $e \in E_{\text {ext }}$ such that (i) $f_{s_{0}}=f_{s_{1}}$ (ii) $\partial_{i} e=v_{s_{i}}$ and (iii) $s_{0}, s_{1}$ and $e$ form a triangle with sides listed in counterclockwise order. We use the notation $\tau=:\left(\partial_{0} \tau, \partial_{1} \tau, e_{\tau}\right)$, and say that $\tau$ points from $\partial_{0} \tau$ to $\partial_{1} \tau$ through $e_{\tau}$.

A dual triangle $\tau=\left(s_{0}, s_{1}, e^{*}\right)$ consists of two sites $s_{i}$ and a dual edge $e^{*} \in E_{\text {ext }}^{*}$ such that (i) $v_{s_{0}}=v_{s_{1}}$ (ii) $\partial_{i} e^{*}=f_{s_{i}}^{*}$ and (iii) $s_{0}, s_{1}$ and $e^{*}$ form a triangle with sides listed in clockwise order. We use the notation $\tau=:\left(\partial_{0} \tau, \partial_{1} \tau, e_{\tau}^{*}\right)$, and say that $\tau$ points from $\partial_{0} \tau$ to $\partial_{1} \tau$ through $e_{\tau}^{*}$.

Each direct (dual) triangle $\tau$ has a complementary triangle $\bar{\tau}$, the unique direct (dual) triangle with $e_{\bar{\tau}}=\bar{e}_{\tau}$.

Two triangles overlap if they 'share part of their area'. Specifically, a dual triangle $\tau$ and a direct triangle $\tau^{\prime}$ overlap if $\partial_{i} \tau=\partial_{i} \tau^{\prime}$ either for $i=0$ or $i=1$, and two triangles of the same type overlap if they are the same triangle. Triangles and their properties are illustrated in Fig 13.

Strips. A (triangle) strip of length $n \geq 0$ is an alternating sequence of sites and triangles $\rho=$ $\left(s_{0}, \tau_{1}, s_{1}, \tau_{2}, \ldots, s_{n-1}, \tau_{n}, s_{n}\right)$ with $\partial_{0} \tau_{i}=s_{i-1}$ and $\partial_{1} \tau_{i}=s_{i}$. We define $\partial_{0} \rho:=s_{0}$ and $\partial_{1} \rho:=s_{n}$. Strips can be given just as a list of sites $\rho=\left(s_{0}, s_{1}, \ldots, s_{n}\right)$ or, if they have non-zero length, as a list of triangles $\rho=\left(\tau_{1}, \ldots, \tau_{n}\right)$. They could also be given as a pair $\rho=(s, \mathbf{x})$, with $s=s_{0}$ the initial site and $\mathbf{x} \in 2^{n}$ a binary vector, because for any given site there exists exactly a dual and a direct triangle pointing from it. Examples of strips are given in Fig 13 .

Let $\rho$ be a strip with $s_{i}=\partial_{i} \rho$. We say that $\rho$ is (i) trivial if it has length zero, (ii) direct (dual) if it consists only of direct (dual) triangles, (iii) proper if it is neither direct nor dual, (iv) open if $v_{s_{0}} \neq v_{s_{1}}$ and $f_{s_{0}} \neq f_{s_{1}}$ and

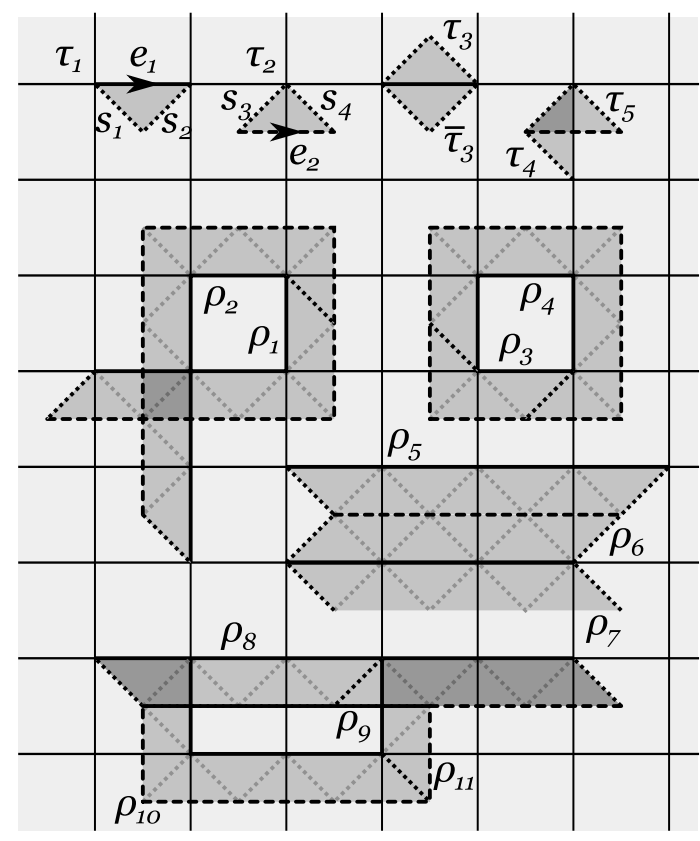

FIG. 13: Several examples of sites, triangles and strips. Sites are displayed as black dotted lines when they are an end of some ribbon. Triangles and strips are displayed as grey bands. The direct path of each strip is a thick black line, and the dual path a dashed line. The $s_{i}$ are sites, the $\tau_{i}$ are triangles and the $\rho_{i}$ strips (indeed ribbons). $\tau_{1}=\left(s_{1}, s_{2}, e_{1}\right)$ is a direct triangle and $\tau_{2}=\left(s_{3}, s_{4}, e_{2}^{*}\right)$ is a dual triangle. $\bar{\tau}_{3}$ is the complementary triangle of $\tau_{3} . \tau_{4}$ and $\tau_{5}$ overlap. $\rho=\rho_{1} \rho_{2}$ is a strip but not a ribbon. $\sigma=\rho_{3} \rho_{4}$ and $\sigma^{\prime}=\rho_{4} \rho_{3}$ are closed strips (indeed ribbons), with $\sigma^{\prime}$ a rotation of $\sigma$ and $\sigma \triangleright$ $\sigma^{\prime}=\rho_{3} . \rho_{5}$ is a dual complementary ribbon or $\rho_{6},\left(\rho_{5}, \rho_{6}\right)_{\triangle}$, and $\rho_{7}$ is direct complementary ribbon of $\rho_{6},\left(\rho_{6}, \rho_{7}\right)_{\nabla}$. The ribbons $\rho_{8}, \rho_{9}, \rho_{10}, \rho_{11}$ illustrate various types of joints, so that $\left(\rho_{8}, \rho_{10}\right)_{\prec,},\left(\rho_{9}, \rho_{11}\right)_{\succ}$ and $\left(\rho_{8} \rho_{9}, \rho_{10} \rho_{11}\right)_{\prec \succ}$.

(v) closed if $s_{0}=s_{1}$. When $\sigma$ is a closed strip we write $\partial \sigma$ instead of $\partial_{i} \sigma$.

Two strips are composable if $\partial_{1} \rho_{1}=\partial_{0} \rho_{2}$. In that case the composed strip is $\rho=\rho_{1} \rho_{2}:=\left(s_{0}, \ldots, s_{m}\right)$ with $\rho_{1}=\left(s_{0}, \ldots, s_{n}\right)$ and $\rho_{2}=\left(s_{n}, \ldots, s_{m}\right)$. This composition operation is clearly associative.

The cyclic nature of the list of triangles of a closed strip allows to rotate it, see Fig 13. We say that $\sigma^{\prime}$ is a rotation of $\sigma$, denoted $\left(\sigma, \sigma^{\prime}\right)_{\circ}$, if $\sigma \neq \sigma^{\prime}, \sigma=\rho_{1} \rho_{2}$ and $\sigma^{\prime}=\rho_{2} \rho_{1}$. In that case we set $\sigma \triangleright \sigma^{\prime}:=\rho_{1}$. Note that if $\left(\sigma, \sigma^{\prime}\right)_{\text {o }}$ then both $\sigma$ and $\sigma^{\prime}$ are closed.

We can attach both a direct and a dual path to a strip $\rho$, see Fig [13. If $\left(\tau_{1}, \ldots, \tau_{q}\right)$ is the ordered list of direct triangles in $\rho$, we set $p_{\rho}=\left(v_{\partial_{0} \tau_{1}}, e_{\tau_{1}}, v_{\partial_{1} \tau_{1}}, \ldots, e_{\tau_{q}}, v_{\partial_{1} \tau_{q}}\right)$. Similarly, if $\left(\tau_{1}^{\prime}, \ldots, \tau_{r}^{\prime}\right)$ is the ordered list of dual triangles in $\rho$, we set $p_{\rho}^{*}=\left(f_{\partial_{0} \tau_{1}^{\prime}}^{*}, e_{\tau_{1}^{\prime}}^{*}, f_{\partial_{1} \tau_{1}^{\prime}}^{*}, \ldots, e_{\tau_{r}^{\prime}}^{*}, f_{\partial_{1} \tau_{r}^{\prime}}^{*}\right)$.

Consider two strips $\rho_{1}$ and $\rho_{2}$. We say that $\rho_{1}$ and $\rho_{2}$ (i) do not overlap, denoted $\left(\rho_{1}, \rho_{2}\right)_{\oslash}$, if no triangle of $\rho_{1}$ overlaps with a triangle of $\rho_{2}$, (ii) form a left joint, denoted $\left(\rho_{1}, \rho_{2}\right)_{\prec}$, if $\rho_{i}=\rho \tau_{i} \rho_{i}^{\prime}$ with $\tau_{1}$ a dual triangle, $\tau_{2}$ a direct triangle and $\left(\rho_{1}^{\prime}, \rho_{2}^{\prime}\right)_{\oslash}$, (iii) form a right joint, 
denoted $\left(\rho_{1}, \rho_{2}\right)_{\succ}$, if $\rho_{i}=\rho_{i}^{\prime} \tau_{i} \rho$ with $\tau_{1}$ a dual triangle, $\tau_{2}$ a direct triangle and $\left(\rho_{1}^{\prime}, \rho_{2}^{\prime}\right)_{\oslash}$, (iv) form a left-right joint, denoted $\left(\rho_{1}, \rho_{2}\right)_{\prec \succ}$, if $\rho_{i}=\rho \tau_{i} \rho_{i}^{\prime} \tau_{i}^{\prime} \rho^{\prime}$ with $\tau_{1}, \tau_{1}^{\prime}$ dual triangles, $\tau_{2}, \tau_{2}^{\prime}$ direct triangles and $\left(\rho_{1}^{\prime}, \rho_{2}^{\prime}\right)_{\oslash}$, and (v) form a crossed joint, denoted $\left(\rho_{1}, \rho_{2}\right)_{+}$, if $\rho_{i}=\rho_{i}^{\prime} \rho_{i}^{\prime \prime}$ with $\left(\rho_{1}^{\prime}, \rho_{2}^{\prime}\right)_{\prec},\left(\rho_{2}^{\prime \prime}, \rho_{1}^{\prime \prime}\right)_{\succ},\left(\rho_{1}^{\prime}, \rho_{2}^{\prime \prime}\right)_{\oslash}$ and $\left(\rho_{1}^{\prime \prime}, \rho_{2}^{\prime}\right)_{\oslash}$. Closed crossed joints are only possible in surfaces of nontrivial topology, see Fig. 15. Indeed, their name was chosen with those cases in which $\rho_{1}, \rho_{2}$ are closed in mind. The other joint types are illustrated in Fig 13 .

We denote by $V_{\rho}\left(F_{\rho}\right)$ the set of vertices (faces) in a strip $\rho$, by $E_{\rho}^{\triangle}\left(E_{\rho}^{\nabla}\right)$ the set of edges $e \in E_{\text {ext }}$ with $e=e_{\tau}$ for some dual (direct) triangle $\tau$ in $\rho$, and by $\bar{E}_{\rho}^{\triangle}$ $\left(\bar{E}_{\rho}^{\nabla}\right)$ the set of their inverses. If $\rho$ and $\rho^{\prime}$ are non-direct (non-dual) closed strips with $E_{\rho}^{\triangle}=\bar{E}_{\rho^{\prime}}^{\triangle}\left(E_{\rho}^{\nabla}=\bar{E}_{\rho^{\prime}}^{\nabla}\right)$, we say that $\rho^{\prime}$ is a dual (direct) complementary ribbon of $\rho$, denoted $\left(\rho, \rho^{\prime}\right) \triangle\left(\left(\rho, \rho^{\prime}\right)_{\nabla}\right)$, see Fig 13.

\section{Ribbons}

Ribbons are strips such that its direct and dual path do not self-cross.

Definition 1 Let $\rho$ be a triangle strip, $p_{\rho}=$ $\left(v_{0}, \ldots, e_{q}, v_{q}\right)$ and $p_{\rho}^{*}=\left(f_{0}^{*}, \ldots, e_{r}^{*}, f_{r}^{*}\right)$. We say that $\rho$ is a ribbon if

- for any triangle $\tau$ in $\rho, \bar{\tau}$ is not in $\rho$,

- for $0 \leq i \neq j \leq q$ with $i \neq 0$ or $j \neq q, v_{i} \neq v_{j}$ and

- for $0 \leq i \neq j \leq r$ with $i \neq 0$ or $j \neq r, f_{i} \neq f_{j}$.

A rotation of a ribbon is a ribbon. Two ribbons $\rho_{1}$ and $\rho_{2}$ are composable if they are composable as strips and $\rho=\rho_{1} \rho_{2}$ is a ribbon. If $\rho$ is a ribbon and $\rho=\rho_{1} \rho_{2}$ as strips, then $\rho_{1}$ and $\rho_{2}$ are ribbons also, and $\left(\rho_{1}, \rho_{2}\right) \oslash$. Complementary ribbons do not overlap.

Consider any two sites $s$ and $s^{\prime}$. If $v_{s}=v_{s^{\prime}}$, we denote by $\alpha_{s, s^{\prime}}$ the unique nontrivial dual ribbon $\rho$ with $\partial_{0} \rho=s$ and $\partial_{1} \rho=s^{\prime}$. If $f_{s}=f_{s^{\prime}}$, we denote by $\beta_{s, s^{\prime}}$ the unique nontrivial direct ribbon $\rho$ with $\partial_{0} \rho=s$ and $\partial_{1} \rho=s^{\prime}$. All nontrivial dual (direct) ribbons take the form $\alpha_{s, s^{\prime}}\left(\beta_{s, s^{\prime}}\right)$ for some $s, s^{\prime}$. We also write $\alpha_{s}:=\alpha_{s, s}$ and $\beta_{s}:=\beta_{s, s}$. Then if $\left(\alpha_{s}, \alpha_{s^{\prime}}\right)$ 。 we have $\alpha_{s} \triangleright \alpha_{s^{\prime}}=\alpha_{s, s^{\prime}}$, and if $\left(\beta_{s}, \beta_{s^{\prime}}\right)$ 。 we have $\beta_{s} \triangleright \beta_{s^{\prime}}=\beta_{s, s^{\prime}}$.

\section{Triangle operators}

From this point on we are working with a fixed finite group $G$. To each edge $e \in E$ we attach a Hilbert space $\mathcal{H}_{G}^{\prime}$ with orthonormal basis $\{|g\rangle\}_{g \in G}$. The total Hilbert space of our system is then $\mathcal{H}_{G}:=\mathcal{H}_{G}^{\prime}{ }^{\otimes|E|}$. If $O$ is a single-qudit operator, $O_{e}$ with $e \in E_{\text {ext }}$ denotes that operator acting on the qudit attached to the edge $e(\bar{e})$ if $e \in E(e \in \bar{E})$.
Before we can define operators for arbitrary ribbons we must consider their elementary components, triangles. To this end, let

$$
L^{h}:=\sum_{g \in G}|h g\rangle\left\langle g\left|, \quad T^{g}:=\right| g\right\rangle\left\langle g\left|, \quad I:=\sum_{g \in G}\right| \bar{g}\right\rangle\langle g| .
$$

If $\tau$ is a dual triangle, we set

$$
L_{\tau}^{h}:=I^{x} L_{e_{\tau}}^{h} I^{x}
$$

with $x=0(x=1)$ if $e_{\tau} \in E\left(e_{\tau} \in \bar{E}\right)$. If $\tau^{\prime}$ is a direct triangle, we set

$$
T_{\tau^{\prime}}^{g}:=I^{x} T_{e_{\tau^{\prime}}}^{g} I^{x}
$$

with $x=0(x=1)$ if $\left.e_{\tau} \in E\left(e_{\tau} \in \bar{E}\right)\right)$. With these definitions we have

$$
\begin{aligned}
L_{\tau}^{h} L_{\tau}^{h^{\prime}}=L_{\tau}^{h h^{\prime}}, & T_{\tau^{\prime}}^{g} T_{\tau^{\prime}}^{g^{\prime}}=\delta_{g, g^{\prime}} T_{\tau^{\prime}}^{g}, \\
L_{\tau}^{h^{\dagger}}=L_{\tau}^{\bar{h}}, \quad & T_{\tau^{\prime}}^{g \dagger}=T_{\tau^{\prime}}^{g}, \\
L_{\tau}^{1}=1, & \sum_{g \in G} T_{\tau^{\prime}}^{g}=1 .
\end{aligned}
$$

As for the commutation rules, we have

$$
L_{\tau}^{h} T_{\tau^{\prime}}^{g}= \begin{cases}T_{\tau^{\prime}}^{h g} L_{\tau}^{h}, & \text { if } \partial_{0} \tau=\partial_{0} \tau^{\prime} \\ T_{\tau^{\prime}}^{g h} L_{\tau}^{h} & \text { if } \partial_{1} \tau=\partial_{1} \tau^{\prime} \\ T_{\tau^{\prime}}^{g} L_{\tau}^{h}, & \text { otherwise }\end{cases}
$$

If $\tau_{1} \neq \tau_{2}$ are dual triangles and $\tau_{1}^{\prime}, \tau_{2}^{\prime}$ are direct triangles then

$$
\left[L_{\tau_{1}}^{h}, L_{\tau_{2}}^{h^{\prime}}\right]=\left[T_{\tau_{1}^{\prime}}^{g}, T_{\tau_{2}^{\prime}}^{g^{\prime}}\right]=0
$$

Thus, non-overlapping triangles have commuting triangle operators.

\section{Ribbon operators}

For each ribbon $\rho$ we introduce a set of operators $\left\{F_{\rho}^{h, g}\right\}$ with $h, g \in G$. We call them ribbon operators. First, if $\epsilon$ is a trivial ribbon we define

$$
F_{\epsilon}^{h, g}:=\delta_{1, g}
$$

If $\tau$ is a dual triangle and $\tau^{\prime}$ a direct triangle we set [18]

$$
F_{\tau}^{h, g}:=\delta_{1, g} L_{\tau}^{h}, \quad F_{\tau^{\prime}}^{h, g}:=T_{\tau^{\prime}}^{g}
$$

If $\rho$ is an arbitrary ribbon of length $l>1$, we let $\rho=\rho_{1} \rho_{2}$ and recursively define a gluing or composition procedure by means of the folowing relations

$$
F_{\rho}^{h, g}:=\sum_{k \in G} F_{\rho_{1}}^{h, k} F_{\rho_{2}}^{\bar{k} h k, \bar{k} g}
$$


We must of course ensure that this definition of $F_{\rho}^{h, g}$ is independent of the particular choice of $\rho_{1}$ and $\rho_{2}$. But this amounts to check that if $\rho=\rho_{1} \rho_{2} \rho_{3}$ then

$$
\sum_{k \in G} F_{\rho_{1}}^{h, k} F_{\rho_{2} \rho_{3}}^{\bar{k} h k, \bar{k} g}=\sum_{k \in G} F_{\rho_{1} \rho_{2}}^{h, k} F_{\rho_{3}}^{\bar{k} h k, \bar{k} g}
$$

which follows by expanding $F_{\rho_{2} \rho_{3}}^{\bar{k} h k, \bar{k} g}$ and $F_{\rho_{1} \rho_{2}}^{h, k}$ with (B9). We also have to check that if $\rho=\epsilon \rho=\rho \epsilon$ then

$$
F_{\rho}^{h, g}=\sum_{k \in G} F_{\epsilon}^{h, k} F_{\rho}^{\bar{k} h k, \bar{k} g}=\sum_{k \in G} F_{\rho}^{h, k} F_{\epsilon^{\prime}}^{\bar{k} h k, \bar{k} g},
$$

which indeed holds true.

We will find useful the notation

$$
T_{\rho}^{g}:=F_{\rho}^{1, g}, \quad L_{\rho}^{h}:=\sum_{g \in G} F_{\rho}^{h, g} .
$$

conceived so that

$$
F_{\rho}^{h, g}=L_{\rho}^{h} T_{\rho}^{g}=T_{\rho}^{g} L_{\rho}^{h} .
$$

Also, for any $S \subset G, g \in G$ we set

$$
F^{S, g}:=\frac{1}{|S|} \sum_{s \in S} F^{s, g}, \quad F^{g, S}:=\sum_{s \in S} F^{g, s} .
$$

We now list several properties of ribbon operators. They follow from the properties of triangle operators and (B9). For any ribbon $\rho$

$$
\begin{gathered}
F_{\rho}^{h, g} F_{\rho}^{h^{\prime}, g^{\prime}}=\delta_{g, g^{\prime}} F_{\rho}^{h h^{\prime}, g}, \quad F_{\rho}^{h, g^{\dagger}}=F_{\rho}^{\bar{h}, g}, \\
L_{\rho}^{1}=\sum_{g \in G} T_{\rho}^{g}=1 .
\end{gathered}
$$

Thus, for each $\rho$, ribbon operators linearly generate an algebra closed under Hermitian conjugation. If $\rho$ is dual and $\rho^{\prime}$ direct then

$$
F_{\rho}^{h, g}=\delta_{g, 1} L_{\rho}^{h}, \quad F_{\rho^{\prime}}^{h, g}=T_{\rho^{\prime}}^{g} .
$$

If $\left(\rho_{1}, \rho_{2}\right)_{\prec}$ then

$$
F_{\rho_{1}}^{h, g} F_{\rho_{2}}^{k, l}=F_{\rho_{2}}^{h k \bar{h}, h l} F_{\rho_{1}}^{h, g} .
$$

If $\left(\rho_{1}, \rho_{2}\right)_{\succ}$ then

$$
F_{\rho_{1}}^{h, g} F_{\rho_{2}}^{k, l}=F_{\rho_{2}}^{k, \bar{g} \bar{h} g} F_{\rho_{1}}^{h, g} .
$$

If $\left(\rho_{1}, \rho_{2}\right)_{\prec \succ}$ then

$$
F_{\rho_{1}}^{h, g} F_{\rho_{2}}^{k, l}=F_{\rho_{2}}^{h k \bar{h}, h l \bar{g} \bar{h} g} F_{\rho_{1}}^{h, g} .
$$

If $\left(\rho_{1}, \rho_{2}\right)_{+}$then

$$
F_{\rho_{1}}^{h, g} F_{\rho_{2}}^{k, l}=F_{\rho_{2}}^{h k \bar{h}, h l} F_{\rho_{1}}^{h, g \bar{l} k l} .
$$

If $\left(\sigma_{1}, \sigma_{2}\right)_{\circ}$ then

$$
F_{\sigma_{1} \triangleright \sigma_{2}}^{k, l} F_{\sigma_{1}}^{h, g}=F_{\sigma_{1} \triangleright \sigma_{2}}^{k h \bar{h} g, l} F_{\sigma_{2}}^{\bar{l} h l, \bar{l} g l} .
$$

If $\left(\rho_{1}, \rho_{2}\right)_{\oslash}$ then

$$
\left[F_{\rho_{1}}^{h, g}, F_{\rho_{2}}^{k, l}\right]=0 .
$$

Proposition 2 Let $\rho$ be a ribbon, $h, g \in G$.

(i) If $\rho$ is dual

$$
\operatorname{Tr}\left(L_{\rho}^{h}\right)=\delta_{h, 1} \operatorname{Tr}(1) .
$$

(ii) If $\rho$ is direct

$$
|G| \operatorname{Tr}\left(T_{\rho}^{g}\right)=\operatorname{Tr}(1) .
$$

(iii) If $\rho$ is proper

$$
|G| \operatorname{Tr}\left(F_{\rho}^{h, g}\right)=\delta_{h, 1} \operatorname{Tr}(1) .
$$

Proof. If $\rho$ is not dual, choose any direct triangle $\tau$ in $\rho$, so that $\rho=\rho_{1} \tau \rho_{2}$. Let $\rho^{\prime}=\rho_{1} \tau^{\prime}$ with $\tau^{\prime}$ dual. Then $\left(\rho^{\prime}, \rho\right)_{\prec}$ and thus using (B18)

$$
\begin{aligned}
\operatorname{Tr}\left(F_{\rho}^{h, g}\right)= & \operatorname{Tr}\left(L_{\rho^{\prime}}^{k} F_{\rho}^{h, g} L_{\rho^{\prime}}^{\bar{k}}\right)= \\
& \operatorname{Tr}\left(F_{\rho}^{k h \bar{k}, k g} L_{\rho^{\prime}}^{k} L_{\rho^{\prime}}^{\bar{k}}\right)=\operatorname{Tr}\left(F_{\rho}^{k h \bar{k}, k g}\right) .
\end{aligned}
$$

If $\rho$ is not direct, choose any dual triangle $\tau$ in $\rho$, so that $\rho=\rho_{1} \tau \rho_{2}$. Let $\rho^{\prime}=\rho_{1} \tau^{\prime}$ with $\tau^{\prime}$ direct. Then $\left(\rho, \rho^{\prime}\right)_{\prec}$ and thus using (B18)

$$
\begin{aligned}
\operatorname{Tr}\left(F_{\rho}^{h, g}\right)= & \sum_{k \in G} \operatorname{Tr}\left(T_{\rho^{\prime}}^{k} F_{\rho}^{h, g} T_{\rho^{\prime}}^{k}\right)= \\
& \sum_{k \in G} \operatorname{Tr}\left(F_{\rho}^{h, g} T_{\rho^{\prime}}^{h k} T_{\rho^{\prime}}^{k}\right)=\delta_{h, 1} \operatorname{Tr}\left(F_{\rho}^{h, g}\right) .
\end{aligned}
$$

Equations (B27, B28) together with (B16, B17) give the desired results.

As a consequence of the previous proposition and B15 we have the following orthogonality results.

Corolary 3 Let $\rho$ be a ribbon, $h, g \in G$.

(i) If $\rho$ is dual

$$
\operatorname{Tr}\left(L_{\rho}^{h^{\dagger}} L_{\rho}^{h^{\prime}}\right)=\delta_{h, h^{\prime}} \operatorname{Tr}(1) .
$$

(ii) If $\rho$ is direct

$$
|G| \operatorname{Tr}\left(T_{\rho}^{g^{\dagger}} T_{\rho}^{g^{\prime}}\right)=\delta_{g, g^{\prime}} \operatorname{Tr}(1) .
$$

(iii) If $\rho$ is proper

$$
|G| \operatorname{Tr}\left(F_{\rho}^{h, g^{\dagger}} F_{\rho}^{h^{\prime}, g^{\prime}}\right)=\delta_{h, h^{\prime}} \delta_{g, g^{\prime}} \operatorname{Tr}(1) .
$$

Definition 4 Rotationally invariant ribbon operators Given a closed ribbon $\sigma$, we say that an operator $F=\sum_{h, g \in G} c_{h, g} F_{\sigma}^{h, g}, c_{h, g} \in \mathbf{C}$, is rotationally invariant if for any $\sigma^{\prime}$ with $\left(\sigma, \sigma^{\prime}\right)$ 。 we have $F=\sum_{h, g \in G} c_{h, g} F_{\sigma^{\prime}}^{h, g}$.

In this regard, results B15 B16, B22) imply that if $\left(\sigma_{1}, \sigma_{2}\right)$ 。 then

$$
F_{\sigma_{1}}^{h, g}=\sum_{l \in G} F_{\sigma_{1} \triangleright \sigma_{2}}^{h \bar{g} \bar{h} g, l} F_{\sigma_{2}}^{\bar{l} h l, \bar{l} g l}
$$




\section{Vertex and face operators}

We now define vertex and face operators in terms of ribbon operators. Let $\alpha_{s}\left(\beta_{s}\right)$ be the unique dual (direct) closed ribbon with $\partial \alpha_{s}=s\left(\partial \beta_{s}=s\right)$. For any site $s=(v, f)$ let

$$
A_{s}^{h}:=F_{\alpha_{s}}^{h, 1} \quad B_{s}^{g}:=F_{\beta_{s}}^{1, \bar{g}} .
$$

Let $s^{\prime}, s^{\prime \prime}$ be sites with $\left(\alpha_{s}, \alpha_{s^{\prime}}\right)_{\circ},\left(\beta_{s}, \beta_{s^{\prime \prime}}\right)_{\circ}$. From (B17. B32) we get

$$
A_{s}^{h}=A_{s^{\prime}}^{h}, \quad B_{s}^{k}=T_{\beta_{s, s^{\prime \prime}}}^{g} B_{s^{\prime \prime}}^{\bar{g} k g} .
$$

Thus, vertex operators are rotationally invariant and we can write $A_{v}^{h}:=A_{s}^{h}$ for $v=v_{s}$. These definitions of $A_{v}^{h}$ and $B_{s}^{g}$ agree with those given in (24).

Let us list several useful properties. If $s \neq s^{\prime}$ then

$$
\begin{gathered}
A_{s}^{h} A_{s}^{h^{\prime}}=A_{s}^{h h^{\prime}}, \quad A_{s}^{1}=1, \quad A_{s}^{h^{\dagger}}=A_{s}^{\bar{h}}, \\
B_{s}^{g} B_{s}^{g^{\prime}}=\delta_{g, g^{\prime}} B_{s}^{g}, \quad \sum_{g \in G} B_{s}^{g}=1, \quad B_{s}^{g^{\dagger}}=B_{s}^{g} . \\
A_{s}^{h} B_{s}^{g}=B_{s}^{h g \bar{h}} A_{s}^{h}, \\
{\left[A_{s}^{h}, B_{s^{\prime}}^{g}\right]=\left[A_{s}^{g}, A_{s^{\prime}}^{g^{\prime}}\right]=\left[B_{s}^{h}, B_{s^{\prime}}^{h^{\prime}}\right]=0 .}
\end{gathered}
$$

All these properties follow from the properties of ribbon operators. Note in particular that the well-known 22] flux metamorphosis B37) is a consequence of $\left(\alpha_{s}, \beta_{s}\right)_{\prec \succ}$.

For subgroups $H, H^{\prime} \subset G, H^{\prime}$ normal, we define the operators

$$
A_{v}^{H}:=A_{s}^{H}:=L_{\alpha_{s}}^{H}, \quad B_{f}^{H^{\prime}}:=B_{s}^{H^{\prime}}:=T_{\beta_{s}}^{H^{\prime}},
$$

where $s=(v, f)$ is a site. We set $A_{v}:=A_{v}^{G}$ and $B_{f}:=$ $B_{f}^{1}$. From (B37,B38) we have for arbitrary vertices $v, v^{\prime}$ and faces $f, f^{\prime}$

$$
\left[A_{v}^{H}, A_{v^{\prime}}^{H}\right]=\left[A_{v}^{H}, B_{f}^{H^{\prime}}\right]=\left[B_{f}^{H^{\prime}}, B_{f^{\prime}}^{H^{\prime}}\right]=0 .
$$

Of particular interest are the commutation rules between ribbon operators and vertex and face operators at their ends. We first consider non-closed ribbons. Let $s_{i}=\partial_{i} \rho$. If $v_{0} \neq v_{1}$ then $\left(\alpha_{s_{0}}, \rho\right)_{\prec},\left(\alpha_{s_{1}}, \rho\right)_{\succ}$ and from (B18, B19) we get

$$
\begin{aligned}
& A_{s_{0}}^{k} F_{\rho}^{h, g}=F_{\rho}^{k h \bar{k}, k g} A_{s_{0}}^{k}, \\
& A_{s_{1}}^{k} F_{\rho}^{h, g}=F_{\rho}^{h, g \bar{k}} A_{s_{1}}^{k} .
\end{aligned}
$$

If $f_{0} \neq f_{1}$ then $\left(\rho, \beta_{s_{0}}\right) \prec,\left(\rho, \beta_{s_{1}}\right) \succ$ and from (B18, B19) we get

$$
\begin{aligned}
& B_{s_{0}}^{k} F_{\rho}^{h, g}=F_{\rho}^{h, g} B_{s_{0}}^{k h}, \\
& B_{s_{1}}^{k} F_{\rho}^{h, g}=F_{\rho}^{h, g} B_{s_{1}}^{\bar{g} \bar{h} g k} .
\end{aligned}
$$

If $\rho$ is dual but not closed then $\alpha_{s_{0}}=\alpha_{s_{0}, s_{1}} \alpha_{s_{1}, s_{0}}$ and from (B9, B15, B23) we get

$$
A_{s_{i}}^{k} F_{\rho}^{h, 1}=F_{\rho}^{k h \bar{k}, 1} A_{s_{i}}^{k},
$$

and if $\rho$ is direct but not closed then $\beta_{s_{0}}=\beta_{s_{0}, s_{1}} \beta_{s_{1}, s_{0}}$ and from (B9, B15, B23) we get

$$
\left[B_{s_{i}}^{k}, F_{\rho}^{1, g}\right]=0 .
$$

Now we consider closed ribbons. So let $\sigma$ be a closed ribbon with $s=\partial \sigma$. If $\sigma$ is a proper closed ribbon then $\left(\alpha_{s}, \sigma\right)_{\prec \succ}$ and $\left(\sigma, \beta_{s}\right)_{\prec \succ}$ so that from (B20) we get

$$
\begin{aligned}
& A_{s}^{k} F_{\sigma}^{h, g}=F_{\sigma}^{k h \bar{k}, k g \bar{k}} A_{s}^{k}, \\
& B_{s}^{k} F_{\sigma}^{h, g}=F_{\sigma}^{h, g} B_{s}^{\bar{g} \bar{h} g k h} .
\end{aligned}
$$

If $\sigma$ is closed but not proper, then either $\sigma=\alpha_{s}$ or $\sigma=\beta_{s}$, but for that case we already have (B37).

Equations (12, 14) can be generalized. Let $\rho$ be a ribbon with two ends $i=0,1$ and set $s_{i}=\partial_{i} \rho, v_{i}=v_{s_{i}}$, $f_{i}=f_{s_{i}}$. If $v_{0} \neq v_{1}$ from (B41) we get

$$
|G| \sum_{g \in G} T_{\rho}^{g} A_{v_{i}} T_{\rho}^{g}=1
$$

and if $f_{0} \neq f_{1}$ from $(\mathrm{B} 42$ we get

$$
\sum_{h \in G} L_{\rho}^{\bar{h}} B_{f_{i}} L_{\rho}^{h}=1 .
$$

As explained in the main text, section II B these identities show how ribbon operators can be used to obtain arbitrary states with a number of excited spots from states with one excited spot less.

\section{Edge operators}

For subgroups $H \subset H^{\prime} \subset G, H$ normal in $G$, we define the operators

$$
L_{e}^{H}:=L_{\tau}^{H}, \quad T_{e^{\prime}}^{H^{\prime}}:=T_{\tau^{\prime}}^{H^{\prime}},
$$

where $e=e_{\tau}, e^{\prime}=e_{\tau^{\prime}}$ with $\tau$ a dual triangle and $\tau^{\prime}$ a direct one. Then from (B5|B6) we have for arbitrary edges $e, e^{\prime}$

$$
\left[L_{e}^{H}, L_{e^{\prime}}^{H}\right]=\left[L_{e}^{H}, T_{e^{\prime}}^{H^{\prime}}\right]=\left[T_{e}^{H^{\prime}}, T_{e^{\prime}}^{H^{\prime}}\right]=0 .
$$

In some particular cases, triangle operators and ribbons have nice commuting properties, but this is not always the case. If $H, H^{\prime}, \tau, \tau^{\prime}$ are as above, with $\tau$ and $\tau^{\prime}$ either in $\rho$ or with no overlap with it then from B9. B15. B23) we have

$$
\left[L_{\tau}^{H}, F_{\rho}^{h, g}\right]=\left[T_{\tau^{\prime}}^{H^{\prime}}, F_{\rho}^{h, g}\right]=0 .
$$

Other triangles are more complicated. Let $\rho=\rho_{1} \rho_{2}$, $\left(\tau_{1}, \rho_{1}\right)_{\succ},\left(\tau_{2}, \rho_{2}\right)_{\prec},\left(\rho_{1}, \tau_{3}\right)_{\succ},\left(\rho_{2}, \tau_{4}\right)_{\prec}, h \in H$ and $k, l \in$ 
$G$ with $k s \bar{k}=s$ for any $s \in H$. Then from B9. B18, B19) we have

$$
\begin{aligned}
L_{\tau_{1}}^{h} F_{\rho}^{k, l} & =\sum_{g \in G} T_{\rho_{1}}^{g} F_{\rho}^{k, g h \bar{g} l} L_{\tau_{1}}^{h}, \\
L_{\tau_{2}}^{h} F_{\rho}^{k, l} & =\sum_{g \in G} T_{\rho_{1}}^{g} F_{\rho}^{k, g \bar{h} \bar{g} l} L_{\tau_{2}}^{h}, \\
T_{\tau_{3}}^{h} F_{\rho}^{k, l} & =\sum_{g \in G} T_{\rho_{1}}^{g} F_{\rho}^{k, l} T_{\tau_{3}}^{h \bar{g} k g}, \\
T_{\tau_{4}}^{h} F_{\rho}^{k, l} & =\sum_{g \in G} T_{\rho_{1}}^{g} F_{\rho}^{k, l} T_{\tau_{4}}^{\bar{g} \bar{k} g h} .
\end{aligned}
$$

As a result, if $N \subset M \subset G$ with $N, M$ normal and $N$ central in $M$ we have for any $h, g \in G, \tau=\tau_{1}$ (or $\tau_{2}$ ) and $\tau^{\prime}=\tau_{3}\left(\right.$ or $\left.\tau_{4}\right)$ with $\tau_{i}$ as above

$$
L_{\tau}^{N} T_{\tau^{\prime}}^{M} F_{\rho}^{h, g} L_{\tau}^{N} T_{\tau^{\prime}}^{M}=\delta_{h M, M} \frac{1}{|N|} F^{h, N g} L_{\tau}^{N} T_{\tau^{\prime}}^{M},
$$

which then gives (58).

\section{The algebra $\mathcal{A}_{\rho}$}

We want to define the ribbon operator algebra $\mathcal{F}_{\rho}$, but as an intermediate step we introduce the algebra $\mathcal{A}_{\rho}$. If $\epsilon$ is a trivial ribbon then $\mathcal{A}_{\epsilon}:=\mathrm{C}$. If $\tau$ is a direct (dual) triangle then $\mathcal{A}_{\tau}:=\operatorname{Lin}\left\{T_{\tau}^{g} \mid g \in G\right\}$ $\left(\mathcal{A}_{\tau}:=\operatorname{Lin}\left\{L_{\tau}^{h} \mid h \in G\right\}\right)$. Finally, if $\rho=\left(\tau_{i}\right)$ is an arbitrary ribbon $\mathcal{A}_{\rho}:=\bigotimes_{i} \mathcal{A}_{\tau_{i}}$. That $\mathcal{A}_{\tau}$ is really an algebra follows from (B4).

We now proceed to show several results which are essential in order to characterize ribbon operator algebras in the next sections.

Lemma 5 Let $\rho$ be a ribbon, $O \in \mathcal{A}_{\rho}$ an operator, $H$ a subgroup of $G$, and $s$ a site

(i) If $\rho$ is not a rotation or a complement of $\alpha_{s}$ then

$$
O A_{s}^{H}=0 \quad \Longrightarrow \quad O=0 .
$$

(ii) If $\rho$ is not a rotation or a complement of $\beta_{s}$ then

$$
O B_{s}^{H}=0 \quad \Longrightarrow \quad O=0 .
$$

(iii) If $\tau$ is a dual triangle such that neither it nor its complement belong to $\rho$

$$
O L_{\tau}^{H}=0 \quad \Longrightarrow \quad O=0 .
$$

(iv) If $\tau$ is a direct triangle such that neither it nor its complement belong to $\rho$

$$
O T_{\tau}^{H}=0 \quad \Longrightarrow \quad O=0 .
$$

Proof. First, note that $A_{s}^{H} A_{s}^{G}=A_{s}^{G}$ implies $O A_{s}^{H}=$ $0 \Rightarrow O A_{s}^{G}=0$, and similarly for $L_{\tau}^{H}$, so that it suffices to consider $H=G$ in these cases. Also, $B_{s}^{H} B_{s}^{1}=B_{s}^{1}$ and similarly for $T_{\tau}^{H}$, so that it suffices to consider $H=1$ for them.

(i) There exists a direct triangle $\tau$ such that it overlaps with $\alpha_{s}$ but not with $\rho$, so that $\left[T_{\tau}^{h}, O\right]=0$, and a site $s^{\prime}$ such that $\left(\alpha_{s^{\prime}}, \alpha_{s}\right)$ 。 and $\left(\tau, \alpha_{s^{\prime}}\right)_{\prec}$ or $\left(\tau, \alpha_{s^{\prime}}\right)_{\succ}$. In the first case $A_{s}^{G}=A_{s^{\prime}}^{G}$ and from (B41) we have $0=$ $\sum_{g} T_{\tau}^{g} O A_{s^{\prime}}^{G} T_{\tau}^{g}=|G|^{-1} \sum_{g, h} O A_{s^{\prime}}^{h} T_{\tau}^{\bar{h}} T_{\tau}^{g}=O A_{s^{\prime}}^{1} T_{\tau}^{G}=$ $O$ and the other case is similar.

(ii) There exists a dual triangle $\tau$ such that it overlaps with $\beta_{s}$ but not with $\rho$, so that $\left[L_{\tau}^{h}, O\right]=0$, and a site $s^{\prime}$ such that $\left(\beta_{s^{\prime}}, \beta_{s}\right)_{\circ}$ and $\left(\beta_{s^{\prime}}, \tau\right)_{\prec}$ or $\left(\beta_{s^{\prime}}, \tau\right)_{\succ}$. In the first case $B_{s}^{1}=B_{s^{\prime}}^{1}$ from (B42) we have $0=\sum_{h} L_{\tau}^{h} O B_{s^{\prime}} L_{\tau}^{\bar{h}}=$ $\sum_{h} O B_{s^{\prime}}^{\bar{h}} L_{\tau}^{h} L_{\tau}^{\bar{h}}=O B_{s^{\prime}}^{G} L_{\tau}^{1}=O$ and the other case is similar.

(iii, iv) The proofs are analogous to (i,ii).

Given a vertex $v$ and a dual triangle $\tau$ we set for any $O \in \mathcal{A}_{\rho}$

$$
O_{k}:=A_{v}^{k} O A_{v}^{\bar{k}}, \quad O_{k}^{\prime}:=L_{\tau}^{k} O L_{\tau}^{\bar{k}} .
$$

One can check that $O_{k}, O_{k}^{\prime} \in \mathcal{A}_{\rho}$. Then if $\rho$ satisfies the conditions of lemma 5

$$
\begin{aligned}
& {\left[O, A_{v}^{H}\right]=0 \quad \Longleftrightarrow \quad|H| O=\sum_{k \in H} O_{k},} \\
& {\left[O, L_{\tau}^{H}\right]=0 \quad \Longleftrightarrow \quad|H| O=\sum_{k \in H} O_{k}^{\prime},}
\end{aligned}
$$

because for $k \in H$ we have $O_{k} A_{v}^{H}=O_{k} A^{k} A_{v}^{H}=$ $A_{v}^{k} O A_{v}^{H}=A^{k} A_{v}^{H} O=A_{v}^{H} O=O A_{v}$ giving $O_{k}=O$, and similarly for $L_{e}^{H}$. As a consequence, we also get under the same conditions and $k \in H$

$$
\left[O, A_{v}^{H}\right]=0 \quad \Longrightarrow \quad\left[O, A_{v}^{k}\right]=0 .
$$

Given a site $s$ in $\rho$ and a direct triangle $\tau$, one can check that unless $\rho$ is both a non-closed and nondirect ribbon with $f_{\partial_{i} \rho}=f_{s}$, for any $O \in \mathcal{A}_{\rho}$ there exist $O_{k, k^{\prime}}, O_{k, k^{\prime}}^{\prime} \in \mathcal{A}_{\rho}$ such that $O=\sum_{k, k^{\prime} \in G} O_{k, k^{\prime}}=$ $\sum_{k, k^{\prime} \in G} O_{k, k^{\prime}}^{\prime}$ with

$$
B_{s}^{g} O_{k, k^{\prime}}=O_{k, k^{\prime}} B_{s}^{\bar{k} g k^{\prime}}, \quad T_{\tau}^{g} O_{k, k^{\prime}}=O_{k, k^{\prime}} T_{\tau}^{\bar{k} g k^{\prime}} .
$$

Then if $\rho$ satisfies the conditions of lemma 5 and $H$ is normal in $G$

$$
\begin{gathered}
{\left[O, B_{f}^{H}\right]=0 \quad \Longleftrightarrow \quad O=\sum_{k, k^{\prime} \in G \mid \bar{k} k^{\prime} \in H} O_{k, k^{\prime}},} \\
{\left[O, T_{\tau}^{H}\right]=0 \quad \Longleftrightarrow \quad O=\sum_{k, k^{\prime} \in G \mid \bar{k} k^{\prime} \in H} O_{k, k^{\prime}}^{\prime}}
\end{gathered}
$$

because $O B_{f}^{H}=O B_{f}^{H} B_{f}^{H}=B_{f}^{H} O B_{f}^{H}=$ $\sum_{k, k^{\prime}} B_{s}^{H} O^{k, k^{\prime}} B_{s}^{H}=\sum_{k, k^{\prime}} O^{k, k^{\prime}} B_{s}^{\bar{k} H k^{\prime}} B_{s}^{H}=$ $\sum_{\bar{k} k^{\prime} \in H} O^{k, k^{\prime}} B_{f}^{H}$ and similarly for $T_{\tau}^{H}$. As a consequence, we also get under the same conditions and $C \in(G / H)_{\text {cj }}$

$$
\left[O, B_{f}^{H}\right]=0 \quad \Longrightarrow \quad\left[O, B_{f}^{C}\right]=0,
$$

where $B_{f}^{C}=\sum_{c \in C} \sum_{h \in H} B_{f}^{c h}$. 


\section{The algebra $\mathcal{F}_{\rho}$}

In this section we characterize the ribbon operator algebra that has been introduced so far.

Definition 6 Let $\rho$ be a ribbon. The ribbon operator algebra $\mathcal{F}_{\rho} \subset \mathcal{A}_{\rho}$ consists of those operators $F \in \mathcal{A}_{\rho}$ such that $\left[F, A_{v}\right]=\left[F, B_{f}\right]=0$ for any vertex $v \neq v_{\partial_{i} \rho}$ and any face $f \neq f_{\partial_{i} \rho}, i=0,1$.

Proposition 7 Let $\rho$ be a ribbon. The $|G|^{2}$ ribbon operators $F_{\rho}^{h, g}, h, g \in G$, linearly generate $\mathcal{F}_{\rho}$. Moreover, $\rho$ is proper if and only if they form a basis of $\mathcal{F}_{\rho}$.

Proof. For ribbons $\rho$ of length zero or one, $\mathcal{F}_{\rho}=\mathcal{A}_{\rho}$ because of (B23) and the first part of the statement follows since ribbon operators generate $\mathcal{A}_{\rho}$. For ribbons of length $l>1$, we proceed inductively on $l$. So let $\rho$ be such a ribbon and set $\rho=: \rho^{\prime} \tau$, with $\tau$ a triangle. Observe that $e_{\tau}$ is not part of $\rho^{\prime}$ and that $\rho^{\prime}$ and $\tau$ share vertices or faces only at their ends, so that $\mathcal{F}_{\rho} \subset \mathcal{F}_{\rho}^{\prime}:=\mathcal{F}_{\rho^{\prime}} \otimes \mathcal{F}_{\tau}=\left(F_{\rho^{\prime}}^{h_{1}, g_{1}} F_{\tau}^{h_{2}, g_{2}} \mid h_{i}, g_{i} \in G\right)$, where $(\cdot)$ is the subspace linearly generated by the set $\cdot$. In view of (B9), what we want to show is that

$$
\mathcal{F}_{\rho}^{\prime \prime}=\left(\sum_{k \in G} F_{\rho^{\prime}}^{h, k} F_{\tau}^{\bar{k} h k, \bar{k} g} \mid h, g \in G\right)
$$

is indeed equal to $\mathcal{F}_{\rho}$. We set $s=\partial_{0} \tau, v=v_{s}, f=f_{s}$ and distinguish two cases.

(a) $\tau$ is direct. In this case, $\mathcal{F}_{\rho} \subset \mathcal{F}_{\rho}^{\prime}$ is the subalgebra of operators commuting with $A_{v}$. Then from(B58) and (B41) we get $\mathcal{F}_{\rho}=\left(\sum_{k \in G} F_{\rho^{\prime}}^{h, k} F_{\tau}^{\bar{k} h^{\prime} k, \bar{k} g} \mid h, h^{\prime}, g \in G\right)$. Applying $F_{\tau}^{h, g}=F_{\tau}^{h^{\prime}, g}$ here and in B65 gives $\mathcal{F}_{\rho}=\mathcal{F}_{\rho}^{\prime \prime}$. (b) $\tau$ is dual. In this case, $\mathcal{F}_{\rho} \subset \mathcal{F}_{\rho}^{\prime}$ with $\mathcal{F}_{\rho}$ the subalgebra of operators commuting with $B_{f}$. Then from(B62) and (B42) we get $\mathcal{F}_{\rho}=\left(F_{\rho^{\prime}}^{h, g} F_{\tau}^{\bar{g} h g, g^{\prime}} \mid h, g, g^{\prime} \in G\right)$. Applying $F_{\tau}^{h, g}=F_{\tau}^{h, 1} \delta_{g, 1}$ here and in (B65) gives $\mathcal{F}_{\rho}=\mathcal{F}_{\rho}^{\prime \prime}$.

This completes the inductive step. The second part of the statement follows from (B17) and corollary 3.

We now construct an alternative basis for $\mathcal{F}_{\rho}$. For each conjugacy class $C \in(G)_{\text {cj }}$ we choose an element $r_{C}$ and denote by $\mathbf{N}_{C} \subset G$ the subgroup of elements commuting with $r_{C}$ and by $Q_{C}$ a set of representatives of $G / \mathbf{N}_{C}$. Then for each $C \in(G)_{\mathrm{cj}}$ we set $C=\left\{c_{i}\right\}_{i=1}^{|C|}$, and $Q_{C}=$ $\left\{q_{i}\right\}_{i=1}^{|C|}$ so that $c_{i}=q_{i} r_{C} \bar{q}_{i}$. Any $g \in G$ can be written in a unique way as $g=q_{i} n$, with $n \in \mathbf{N}_{C}$. We introduce index functions as follows: $i(g):=i$ and $n(g):=n$. For each irreducible representation $R \in\left(\mathbf{N}_{C}\right)_{\text {ir }}$, we choose a particular basis and denote by $\Gamma_{R}(k), k \in G$, the corresponding unitary matrices of the representation. The desired new basis is the following:

$$
F_{\rho}^{R C ; \mathbf{u v}}:=\frac{n_{R}}{\left|\mathbf{N}_{C}\right|} \sum_{n \in \mathbf{N}_{C}} \bar{\Gamma}_{R}^{j j^{\prime}}(n) F_{\rho}^{\bar{c}_{i}, q_{i} n \bar{q}_{i^{\prime}}}
$$

where $\mathbf{u}=(i, j), \mathbf{v}=\left(i^{\prime}, j^{\prime}\right)$ with $i, i^{\prime}=1, \ldots,|C|$ and $j, j^{\prime}=1, \ldots, n_{R}$. The inverse change is

$$
F_{\rho}^{h, g}=\sum_{R \in\left(\mathbf{N}_{C}\right)_{\mathrm{ir}}} \sum_{j, j^{\prime}=1}^{n_{R}} \Gamma_{R}^{j j^{\prime}}\left(n_{h, g}\right) F_{\rho}^{R C ; \mathbf{u v}}
$$

where $\bar{h} \in C \in(G)_{\mathrm{cj}}, n_{h, g}=\bar{q}_{i(\bar{h})} g q_{i(\bar{g} \bar{h} g)}, \mathbf{u}=(i(\bar{h}), j)$ and $\mathbf{v}=\left(i(\bar{g} \bar{h} g), j^{\prime}\right)$ using the index functions for $C$. That (B66) is really a basis follows from

$$
\begin{aligned}
\operatorname{Tr}\left(F_{\rho}^{R C ; \mathbf{u v}^{\dagger}} F_{\rho}^{R^{\prime} C^{\prime} ; \mathbf{u}^{\prime} \mathbf{v}^{\prime}}\right)= \\
\quad=\frac{\left|n_{R}\right|}{\left|\mathbf{N}_{C}\right||G|} \delta_{R, R^{\prime}} \delta_{C, C^{\prime}} \delta_{\mathbf{u}, \mathbf{u}^{\prime}} \delta_{\mathbf{v}, \mathbf{v}^{\prime}} \operatorname{Tr}(1)
\end{aligned}
$$

Instead of B41, B42 we can now write for $D_{s}^{h, g}:=$ $A_{s}^{h} B_{s}^{g}, s_{i}=\partial_{i} \rho$,

$$
\begin{aligned}
& D_{s_{0}}^{h, g} F^{R C ; \mathbf{u v}}=\sum_{s=1}^{n_{R}} \Gamma_{R}^{s j}\left(n\left(h q_{i}\right)\right) F^{R C ; \mathbf{u}(s) \mathbf{v}} D_{s_{0}}^{h, g \bar{c}_{i}}, \\
& D_{s_{1}}^{h, g} F^{R C ; \mathbf{u v}}=\sum_{s=1}^{n_{R}} \bar{\Gamma}_{R}^{s j^{\prime}}\left(n\left(h q_{i^{\prime}}\right)\right) F^{R C ; \mathbf{u v}(s)} D_{s_{1}}^{h, c_{i^{\prime}} g},
\end{aligned}
$$

where $\mathbf{u}=(i, j), \mathbf{v}=\left(i^{\prime}, j^{\prime}\right), \mathbf{u}(s)=\left(i\left(h q_{i}\right), s\right), \mathbf{v}(s)=$ $\left(i\left(h q_{i^{\prime}}\right), s\right)$.

\section{The algebra $\mathcal{K}_{\sigma}$}

Here we discuss the algebra of operators that gives the projectors onto states of different topological charge in systems with Hamiltonian $H_{G}$ (1).

Definition 8 Let $\sigma$ be a closed ribbon. The closed ribbon operator algebra $\mathcal{K}_{\sigma} \subset \mathcal{A}_{\sigma}$ consists of those operators $K \in \mathcal{A}_{\sigma}$ such that $\left[K, A_{v}\right]=\left[K, B_{f}\right]=0$ for every vertex $v$ and face $f$.

Note that $\mathcal{K}_{\sigma} \subset \mathcal{F}_{\sigma}$. It is not difficult to check that $\mathcal{K}_{\alpha_{s}}$ is linearly generated by the operators $A_{s}^{h}, h \in G$, and $\mathcal{K}_{\beta_{s}}$ is linearly generated by the operators $B_{s}^{C}$, with $C \in(G)_{\mathrm{cj}}$ and $B_{s}^{C}=\sum_{g \in C} B_{s}^{g}$. Note that these are exactly the rotationally invariant subalgebras of $\mathcal{F}_{\alpha_{s}}$ and $\mathcal{F}_{\beta_{s}}$.

For any closed ribbon $\sigma$ we define the operators

$$
K_{\sigma}^{D C}:=\sum_{q \in Q_{C}} \sum_{d \in D} F_{\sigma}^{q d \bar{q}, q r_{C} \bar{q}}
$$

where $C \in(G)_{\mathrm{cj}}$ and $D \in\left(\mathbf{N}_{C}\right)_{\mathrm{cj}}$. The point of these operators is that they are rotationally invariant:

$$
\left(\sigma, \sigma^{\prime}\right)_{\circ} \quad \Longrightarrow \quad K_{\sigma^{\prime}}^{D C}=K_{\sigma}^{D C}
$$

as can be checked applying (B32). In fact, it can be shown that if $\sigma$ is proper they form a basis of the subalgebra of rotationally invariant ribbon operators of $\mathcal{F}_{\sigma}$. 
From (B15, B16) we get

$$
\begin{aligned}
K_{\sigma}^{D C} K_{\sigma}^{D^{\prime} C^{\prime}} & =\delta_{C, C^{\prime}} \sum_{D^{\prime \prime}} N_{D D^{\prime}}^{D^{\prime \prime}} K_{\sigma}^{D^{\prime \prime} C}, \\
K_{\sigma}^{D C^{\dagger}} & =K_{\sigma}^{\bar{D} C}, \\
\sum_{C \in(G)_{c \mathrm{j}}} K_{\sigma}^{1 C} & =1 .
\end{aligned}
$$

where the sum runs over $D^{\prime \prime} \in\left(\mathbf{N}_{C}\right)_{\mathrm{cj}}, D D^{\prime}=$ $\sum_{D^{\prime \prime}} N_{D, D^{\prime}}^{D^{\prime \prime}} D^{\prime \prime}$ and $\bar{D}$ denotes the inverse class of $D$. The result (B26) implies for any proper $\sigma$

$$
|G| \operatorname{Tr}\left(K_{\sigma}^{D C}\right)=|C| \delta_{D, 1} \operatorname{Tr}(1),
$$

which together with $(\underline{\mathrm{B} 72})$ and $N_{D D^{\prime}}^{1}=\delta_{\bar{D}, D^{\prime}}|D|$ gives

$$
\operatorname{Tr}\left(K_{\sigma}^{D C^{\dagger}} K_{\sigma}^{D^{\prime} C^{\prime}}\right)=\frac{|D||C|}{|G|} \delta_{D, D^{\prime}} \delta_{C, C^{\prime}} \operatorname{Tr}(1) .
$$

Proposition 9 Let $\sigma$ be a proper closed ribbon. The operators $K_{\sigma}^{D C}, C \in(G)_{\mathrm{cj}}, D \in\left(\mathbf{N}_{C}\right)_{\mathrm{cj}}$, form a basis of $\mathcal{K}_{\sigma}$.

Proof. This is just a particular case of proposition 14

For any proper closed ribbons $\sigma$, consider the subalgebra $\mathcal{K}_{\sigma}^{C} \subset \mathcal{K}_{\sigma}$ with basis $\left\{K_{\sigma}^{D C} \mid D \in\left(\mathbf{N}_{C}\right)_{\mathrm{cj}}\right\}$. The point is that in view of $\mathrm{B} 72$, A21) we have $\mathcal{K}_{\sigma}^{C} \simeq \mathcal{Z}_{C}$, where $\mathcal{Z}_{C}$ is the center of the group algebra $\mathbf{C}\left[\mathbf{N}_{C}\right]$. In particular the isomorphism identifies $K^{D C}$ with $e_{D}:=$ $\sum_{h \in D} h$. Note that the isomorphism preserves adjoints as defined in (A18). This suggests the introduction of a different basis for $\mathcal{K}_{\sigma}$. We define in analogy with (A24)

$$
K_{\sigma}^{R C}:=\frac{n_{R}}{\left|\mathbf{N}_{C}\right|} \sum_{D \in\left(\mathbf{N}_{C}\right)_{c j}} \bar{\chi}_{R}(D) K_{\sigma}^{D C},
$$

where $R \in\left(\mathbf{N}_{C}\right)_{\text {ir. }}$. Due to A25, the reverse change of basis is:

$$
K_{\sigma}^{D C}=\sum_{R \in\left(\mathbf{N}_{C}\right)_{\mathrm{ir}}} \frac{|D|}{n_{R}} \chi_{R}(D) K_{\sigma}^{R C} .
$$

And due to A23), the elements of the new basis are orthogonal projectors summing up to the identity:

$$
\begin{aligned}
K_{\sigma}^{R C^{\dagger}} & =K_{\sigma}^{R C}, \\
K_{\sigma}^{R C} K_{\sigma}^{R^{\prime} C^{\prime}} & =\delta_{R, R^{\prime}} \delta_{C, C^{\prime}} K_{\sigma}^{R C}, \\
\sum_{R, C} K_{\sigma}^{R C} & =1 .
\end{aligned}
$$

\section{The algebra $\mathcal{J}_{\rho}$}

We discuss now the algebra of operators that gives the projectors onto states with different domain wall types in systems with Hamiltonian $H_{G}^{N M}$ (35), with $N \subset M \subset G$ subgroups, $N$ normal in $G$.
Definition 10 Let $\rho$ be an open ribbon. The ribbon operator algebra $\mathcal{J}_{\rho} \subset \mathcal{F}_{\rho}$ consists of those operators $J \in \mathcal{F}_{\rho}$ such that $\left[J, A_{v}^{M}\right]=\left[J, B_{f}^{N}\right]=0$ for every vertex $v$ and face $f$.

We denote by $r_{T}$ an arbitrarily chosen representative of a class $T$ of the double coset $M \backslash G / M$, by $\mathbf{N}_{T} \subset M$ the subgroup of elements $m$ such that $m r_{T} M=r_{T} M$ and by $Q_{T}$ a set of representatives of $M / \mathbf{N}_{T}$. For any open ribbon $\rho$ we define the operators

$$
J_{\rho}^{C T}:=\sum_{q \in Q_{T}} \sum_{c \in C} F_{\rho}^{q c \bar{q}, q r_{T} M},
$$

where $C \in\left(N, \mathbf{N}_{T}\right)_{\mathrm{cj}}$ and $T \in M \backslash G / M$. From (B15) we get

$$
\begin{aligned}
J_{\rho}^{C T} J_{\rho}^{C^{\prime} T^{\prime}} & =\delta_{T, T^{\prime}} \sum_{C^{\prime \prime}} N_{C C^{\prime}}^{C^{\prime \prime}} J_{\rho}^{C^{\prime \prime} T}, \\
J_{\rho}^{C T^{\dagger}} & =J_{\rho}^{\bar{C} T} \\
\sum_{T \in M \backslash G / M} J_{\rho}^{1 T} & =1,
\end{aligned}
$$

where the sum runs over $C^{\prime \prime} \in(N, M)_{\mathrm{cj}}, C C^{\prime}=$ $\sum_{C^{\prime \prime}} N_{C, C^{\prime}}^{C^{\prime \prime}} C^{\prime \prime}$ and $\bar{C}$ denotes the inverse class of $C$. From (B26) we get

$$
|G| \operatorname{Tr}\left(J_{\rho}^{C T}\right)=|T| \delta_{C, 1} \operatorname{Tr}(1),
$$

which together with (B79) and $N_{C C^{\prime}}^{1}=\delta_{\bar{C}, C^{\prime}}|C|$ gives

$$
|G| \operatorname{Tr}\left(J_{\rho}^{C T^{\dagger}} J_{\rho}^{C^{\prime} T^{\prime}}\right)=|C||T| \delta_{C, C^{\prime}} \delta_{T, T^{\prime}} \operatorname{Tr}(1)
$$

Proposition 11 Let $\rho$ be an open ribbon. The operators $J_{\rho}^{C T}, C \in\left(N, \mathbf{N}_{T}\right)_{\mathrm{cj}}, T \in M \backslash G / M$, form a basis of $\mathcal{J}_{\rho}$.

Proof. Set $\left(v_{i}, f_{i}\right)=\partial_{i} \rho$ and let $\mathcal{F}_{\rho}^{\prime} \subset \mathcal{F}_{\rho}$ be the subalgebra of operators commuting with $A_{v_{i}}^{M}$. From (B41) and (B58) we get $\mathcal{F}_{\rho}^{\prime}=\left(\sum_{m \in M} F_{\rho}^{m h \bar{m}, m g M} \mid h, g \in G\right) . \mathcal{J}_{\rho} \subset$ $\mathcal{F}_{\rho}^{\prime}$ is the subalgebra of operators commuting with $B_{f_{i}}^{N}$. From (B42, B62) we get $\mathcal{J}_{\rho}=\left(\sum_{m \in M} F_{\rho}^{m h \bar{m}, m g M} \mid h \in\right.$ $N, g \in G)$. Finally, if $h \in C \in\left(N, \mathbf{N}_{T}\right)_{\text {cj }}$ and $g \in T \in$ $M \backslash G / M$ we have $\sum_{m, \in M} F_{\rho}^{m h \bar{m}, m g M}=\frac{\left|\mathbf{N}_{T}\right|}{|C|} J_{\rho}^{C T}$. The result follows in view of (B81).

From the previous proposition and (B9, B51, B52) we get the following result, which is no longer true if the condition of $N$ being abelian is removed.

Corolary 12 Let $\rho$ be an open ribbon and e an edge. If $N$ is abelian $\left[J, L_{e}^{N}\right]=\left[J, T_{e}^{M}\right]=0$ for any $J \in \mathcal{J}_{\rho}$.

For any open ribbon $\rho$ and $T \in M \backslash G / M$, consider the subalgebra $\mathcal{J}_{\rho}^{T} \subset \mathcal{J}_{\rho}$ with basis $\left\{J_{\rho}^{C T} \mid C \in\left(N, \mathbf{N}_{T}\right)_{\mathrm{cj}}\right\}$. The point is that in view of A21, B79 we have $\mathcal{J}_{C} \simeq$ $\mathcal{Z}_{N, \mathbf{N}_{T}}$. In particular the isomorphism identifies $J^{C T}$ with $e_{C}^{M}$. Note that the isomorphism preserves adjoints 
as defined in (A18). This suggests the introduction of a different basis for $\mathcal{J}_{\rho}$. We define in analogy with (A30),

$$
J_{\rho}^{R T}:=\frac{n_{R}|\tilde{R}||N|}{\left|\mathbf{N}_{T}\right|^{2}} \sum_{C \in\left(N, \mathbf{N}_{T}\right)_{\mathrm{cj}}} \bar{\chi}_{R}(C) J_{\rho}^{C T},
$$

where $R \in\left(N, \mathbf{N}_{T}\right)_{\text {ir }}$. Due to (A31), the reverse change of basis is:

$$
J_{\rho}^{C T}=\sum_{R \in\left(N, \mathbf{N}_{T}\right)_{\mathrm{ir}}} \frac{|C|}{n_{R}} \chi_{R}(C) J_{\rho}^{R T}
$$

And due to A29, the elements of the new basis are orthogonal projectors summing up to the identity:

$$
\begin{aligned}
J_{\rho}^{R T^{\dagger}} & =J_{\rho}^{R T}, \\
J_{\rho}^{R T} J_{\rho}^{R^{\prime} T^{\prime}} & =\delta_{R, R^{\prime}} \delta_{T, T^{\prime}} J_{\rho}^{R T}, \\
\sum_{R, T} J_{\rho}^{R T} & =1 .
\end{aligned}
$$

Two comments should be made here. First, in the particular case of $M$ normal in $G, M \backslash G / M=G / M$ and for $T \in G / M$ we have $\mathbf{N}_{T}=M$, so that the two labels for the basis of $\mathcal{J}_{\rho}$ are not related anymore. Secondly, although definition 10 only applies to open ribbons, the algebra $\mathcal{J}_{\rho}$ can be extended to any $\rho$ taking proposition 11 as a definition. As long as $\rho$ is proper, the properties B79, B84 will still hold.

The special case of $M$ normal and $N$ central in $M$ deserves special attention. Instead of (B78B82 we can write

$$
J_{\rho}^{n, t}:=F^{n, t M}, \quad J_{\rho}^{\chi, t}:=\frac{1}{|N|} \sum_{n \in N} \bar{\chi}(n) J_{\rho}^{n, t},
$$

where $n \in N, \tilde{t} \in G / M$ and $\chi \in(N)_{\mathrm{ch}}$, with $(N)_{\mathrm{ch}}$ the character group of $N$. Then, if $\rho=\rho_{1} \rho_{2}$ is an open ribbon from (B9) we get 64).

\section{The algebra $\mathcal{K}_{\sigma}^{\prime}$}

Here we discuss the algebra of operators that gives the projectors onto states of different charge, confined and topological, in systems with Hamiltonian $H_{G}^{N M}$, 35. where $N \subset M \subset G$ are normal subgroups in $G$ with $N$ central in $M$.

Definition 13 Let $\sigma$ be a closed ribbon. The closed ribbon operator algebra $\mathcal{K}_{\sigma}^{\prime} \subset \mathcal{F}_{\sigma}$ consists of those operators $K \in \mathcal{F}_{\sigma}$ such that $\left[K, A_{v}^{M}\right]=\left[K, B_{f}^{N}\right]=\left[K, T_{e}^{M}\right]=$ $\left[K, L_{e}^{N}\right]=0$ for any vertex $v$, face $f$ and edge $e$.

Note that if $N=1$ and $M=G$ then $\mathcal{K}_{\sigma}^{\prime}=\mathcal{K}_{\sigma}$. We extend our previous notation and set $(A, B)_{\mathrm{cj}}:=$ $\{\{b a \bar{b} \mid b \in B\} \mid a \in A\}$ for two subgroups $A, B$ of some other group. For each class $C \in(G / N, M / N)_{\mathrm{cj}}$, we choose a representative $r_{C} \in G$. Let $\mathbf{N}_{C}^{\prime}:=\{m \in$ $\left.M \mid m r_{C} \bar{m} \bar{r}_{C} \in N\right\}$ and choose a set $Q_{C} \subset M$ of representatives of $M / \mathbf{N}_{C}^{\prime}$. For any closed ribbon $\sigma$ we define the operators

$$
K_{\sigma}^{D C}:=\sum_{q \in Q_{C}} \sum_{d \in D} \sum_{n \in N} F_{\sigma}^{q d \bar{q}, q r_{C} \bar{q} n}
$$

where $C \in(G / N, M / N)_{\text {cj }}$ and $D \in\left(\mathbf{N}_{C}^{\prime}\right)_{\text {cj }}$. With this notation, the results (B72) remain true, and (B73, B88) only need a slight modification:

$$
\begin{aligned}
|G| \operatorname{Tr}\left(K_{\sigma}^{D C}\right) & =|C||N| \delta_{D, 1} \operatorname{Tr}(1), \\
\operatorname{Tr}\left(K_{\sigma}^{D C^{\dagger}} K_{\sigma}^{D^{\prime} C^{\prime}}\right) & =\frac{|D||C||N|}{|G|} \delta_{D, D^{\prime}} \delta_{C, C^{\prime}} \operatorname{Tr}(1) .
\end{aligned}
$$

Proposition 14 Let $\sigma$ be a proper closed ribbon. The operators $K_{\sigma}^{D C}, C \in(G / N, M / N)_{\mathrm{cj}}, D \in\left(\mathbf{N}_{C}^{\prime}\right)_{\mathrm{cj}}$, form a basis of $\mathcal{K}_{\sigma}^{\prime}$.

Proof. Set $(v, f)=\partial \sigma$ and let $\mathcal{F}_{\sigma}^{\prime} \subset \mathcal{F}_{\sigma}$ be the subalgebra of operators commuting with $A_{v}^{M}$ and $B_{f}^{N}$. From (B45, B58, B62) we get $\mathcal{F}_{\sigma}^{\prime}=\left(\sum_{m \in M} F_{\sigma}^{\bar{m} h m, \bar{m} g m} \mid h, g \in\right.$ $G, h g \bar{h} \bar{g} \in N) . \quad \mathcal{K}_{\sigma}^{\prime} \subset \mathcal{F}_{\sigma}^{\prime}$ is the subalgebra of operators commuting with $L_{e}^{N}$ and $T_{e}^{M}$ for every edge e. From (B50, B51, B59, B63) it follows that $\mathcal{K}_{\sigma}=$ $\left(\sum_{m \in M} \sum_{n \in N} F_{\sigma}^{m h \bar{m}, m g \bar{m} n} \mid h \in M, g \in G, h g \bar{h} \bar{g} \in\right.$ $N)$. But given such $h$ and $g$ there exists a class $C \in(G / N, M / N)_{\text {cj }}$ and $q \in Q_{C}$ with $\bar{q} g q \bar{r}_{C} \in N$, and a class $D \in\left(\mathbf{N}_{C}^{\prime}\right)_{\text {cj }}$ with $\bar{q} h q \in D$, so that $\sum_{m \in M} \sum_{n \in N} F_{\sigma}^{m h \bar{m}, m g \bar{m} n}=\frac{\left|\mathbf{N}_{C}^{\prime}\right|}{D \mid} K_{\sigma}^{D C}=\frac{|M|}{|C||D|} K_{\sigma}^{D C}$. The result follows in view of $(\underline{\mathrm{B} 88})$.

The change of basis B75 that leads to the relations (B77) is possible for $\mathcal{K}_{\sigma}^{\prime}$ just as it was for $\mathcal{K}_{\sigma}$, with the only difference that now the representations $R$ belong to $\left(\mathbf{N}_{C}^{\prime}\right)_{\text {ir. }}$. fact

For any proper closed ribbon we have $\mathcal{J}_{\sigma} \subset \mathcal{K}_{\sigma}^{\prime}$. In

$$
J_{\sigma}^{n t}=\sum_{C \subset t M} K_{\sigma}^{n C}
$$

where $n \in N, t \in G / M, C \in(G / N, M / N)_{\mathrm{cj}}$ and $K_{\sigma}^{n C}=K_{\sigma}^{D C}$ with $D=\{n\} \in\left(N, \mathbf{N}_{C}^{\prime}\right)_{\mathrm{cj}} \subset\left(\mathbf{N}_{C}^{\prime}\right)_{\mathrm{cj}}$. From (B76, B82, B89) we get the following relation between the corresponding projector bases

$$
J_{\sigma}^{\chi t}=\sum_{C \subset t M} \sum_{R \in\left(\mathbf{N}_{C}^{\prime}\right)_{\mathrm{ir}}} \frac{\left(\chi_{R}, \chi\right)_{N}}{n_{R}} K_{\sigma}^{R C},
$$

where $\chi \in(N)_{\mathrm{ch}}, t \in G / M$ and $C \in(G / N, M / N)_{\mathrm{cj}}$.

\section{APPENDIX C: RIBBON TRANSFORMATIONS}

In this appendix we discuss several transformations that can be applied to ribbons. These transformations are interesting because they leave invariant the action of 


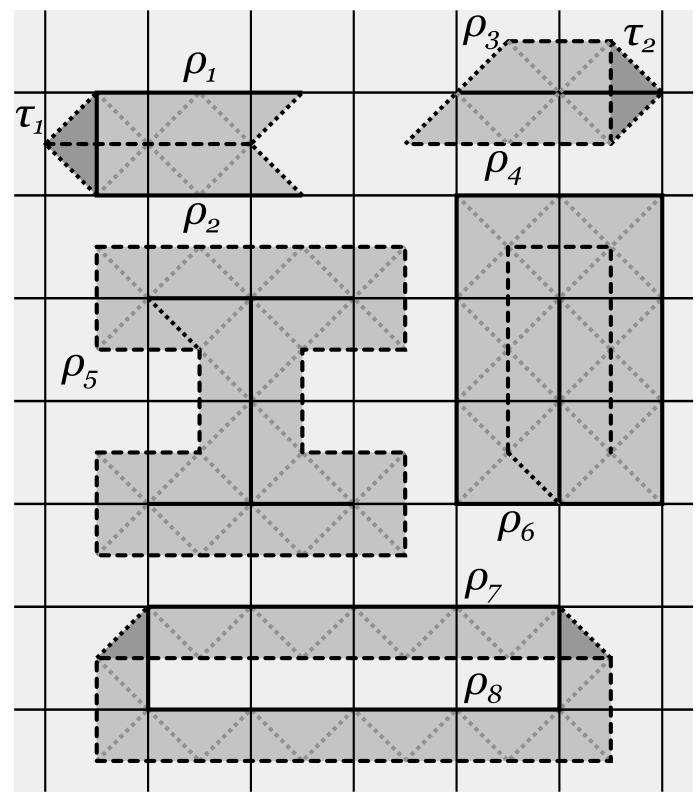

FIG. 14: Several constructions with nice strips and ribbons. All elements are displayed as in Fig. 13 The $\rho_{i}$ are ribbons, except $\rho_{5}$ and $\rho_{6}$ which are just nice strips. We have $\left(\rho_{1}, \tau_{1}, \rho_{2}\right)_{\triangleleft}$ and $\left(\rho_{3}, \tau_{2}, \rho_{4}\right)_{\triangleright} . \rho_{5}$ is a dual block and $\rho_{6}$ is a direct block. $\rho_{7}$ and $\rho_{8}$ form a simple deformation, $\left(\rho_{7}, \rho_{8}\right)_{=}$.

certain ribbon operator algebras on suitable subspaces of $\mathcal{H}_{G}$. In order to proof the desired properties, we need some preliminary results. We will find useful the notation $\cdot={ }_{\psi} \cdot$ for $\cdot|\psi\rangle=\cdot|\psi\rangle$. Also, for strips $\rho, \rho^{\prime}$ and a triangle $\tau$, we write $\left(\rho, \tau, \rho^{\prime}\right)_{\triangleleft}$ if $\tau$ is direct, $(\rho, \tau)_{\prec,}\left(\rho^{\prime}, \tau\right)_{\succ}$ and we write $\left(\rho, \tau, \rho^{\prime}\right)_{\triangleright}$ if $\tau$ is dual, $(\tau, \rho)_{\prec},\left(\tau, \rho^{\prime}\right)_{\succ}$, see Fig. 14.

Lemma 15 Let $\rho, \rho^{\prime}$ be ribbons, $|\psi\rangle \in \mathcal{H}_{G}$ and $H, H^{\prime} \subset$ $G$ normal subgroups with $h h^{\prime}=h^{\prime} h$ for any $h \in H, h^{\prime} \in$ $H^{\prime}$.

(i) If $\left(\rho, \rho^{\prime}\right) \triangle$, there exist a direct triangle $\tau$ such that $\left(\rho, \tau, \rho^{\prime}\right)_{\triangleleft}$ and for any $f \in F_{\rho}-\left\{f_{\partial_{0} \rho}, f_{\partial_{1} \rho}\right\}$ we have $B_{f}^{H}={ }_{\psi} 1$ then for $h^{\prime} \in H^{\prime}$

$$
L_{\rho}^{h^{\prime}}={ }_{\psi} \sum_{k, l \in G} F_{\rho^{\prime}}^{l \bar{k} \bar{h}^{\prime} k \bar{l}, l_{T}} T_{\tau}^{k} .
$$

(ii) If $\left(\rho, \rho^{\prime}\right)_{\nabla}$ then for $g \in G$

$$
T_{\rho}^{g}={ }_{\psi} T_{\rho^{\prime}}^{\bar{g}}
$$

Proof. (i) Let $p_{\rho}^{*}=\left(f_{0}^{*}, \ldots, e_{r}^{*}, f_{r}^{*}\right)$ and set $s_{i}=$ $\left(\partial_{0} e_{i+1}, f_{i}\right)$ for $i=1, \ldots, r-1$. Consider the states $\left|\psi^{g, \mathbf{h}}\right\rangle:=L_{e_{1}}^{H^{\prime}} T_{\tau}^{g} L_{e_{2}}^{H^{\prime}} B_{s_{1}}^{h_{1}} \cdots L_{e_{r}}^{H^{\prime}} B_{s_{r-1}}^{h_{r-1}}|\psi\rangle, g \in G, \mathbf{h} \in$ $H^{r-1}$. Then $L_{e_{i}}^{H^{\prime}}={ }_{\psi^{g, \mathbf{h}}} 1$ and thus $L_{\rho}^{H^{\prime}}={ }_{\psi^{g, \mathbf{h}}} L_{\rho^{\prime}}^{H^{\prime}}={ }_{\psi^{g, \mathbf{h}}}$ 1. But $|\psi\rangle=\sum_{g \in G} T_{\tau}^{g} \sum_{\mathbf{h} \in H^{r-1}} \prod_{i=1}^{r-1} B_{s_{i-1}}^{h_{i}}|\psi \mathbf{g}\rangle$, and the result follows using (B51) and the fact that $\left[L_{\rho}^{k}, B_{s_{i}}^{h}\right]=\left[L_{\rho^{\prime}}^{k}, B_{s_{i}}^{h}\right]=0$ for $k \in H^{\prime}, h \in H$.

(ii) The proof is dual to (i). Just note that one must use $\sum_{k \in G} L_{\tau}^{\bar{k}} T_{e_{1}}^{1} L_{\tau}^{k}=1$ instead of $\sum_{k \in G} T_{\tau}^{k} L_{e_{1}}^{H^{\prime}} T_{\tau}^{k}=1$ and $\sum_{k \in G} A_{v_{i-1}}^{\bar{k}} T_{e_{i}}^{1} A_{v_{i-1}}^{k}=1$ instead of $\sum_{k \in H} B_{f_{i-1}}^{k} L_{e_{i}}^{H^{\prime}} B_{s_{i-1}}^{k}=B_{f_{i-1}}^{H}$. Alternatively, the result is trivial in terms of Wilson loops.

When working with ribbon deformations, it is useful to consider triangle strips that are more general than ribbons but still allow to introduce operators. We say that a strip $\rho$ is nice if no two of its triangles overlap or, equivalently, if $\rho=\rho_{1} \cdots \rho_{n}$ with $\rho_{i}$ ribbons such that $\left(\rho_{i}, \rho_{j}\right)_{\oslash}$ for $i \neq j$. Then ribbon operators can be generalized for nice strips using (B9). Although such nice strip operators still commute with all vertex operators $A_{v}$ and face operators $B_{f}$ except those in their ends, they can no longer be characterized by this property. A direct (dual) block $\rho$ is a nice closed strip such that $(\rho, \rho)_{\triangle}$ $\left((\rho, \rho)_{\nabla}\right)$, see Fig. 14.

Lemma 16 Let $\rho$ be a nice closed strip, $|\psi\rangle \in \mathcal{H}_{G}$ and $H \subset G$ a normal subgroup.

(i) If $\rho$ is a dual block and for any $v \in V_{\rho}$ we have $A_{v}^{H}={ }_{\psi}$ 1 then

$$
L_{\rho}^{H}={ }_{\psi} 1
$$

(ii) If $\rho$ is a direct block and for any $f \in F_{\rho}$ we have $B_{f}^{H}={ }_{\psi} 1$ then

$$
T_{\rho}^{H}={ }_{\psi} 1
$$

Proof. (i) We proceed recursively on $\left|V_{\rho}\right|$. For $\left|V_{\rho}\right|=$ 0,1 the result is trivial. So let $\left|V_{\rho}\right|>1$. Note that if $\left(\rho, \rho^{\prime}\right)_{\circ}$ then $L_{\rho}^{H}=L_{\rho^{\prime}}^{H}$ due to (B32). Also, the path $p_{\rho}=\left(v_{0}, \ldots, e_{q}^{\prime}, v_{r}\right)$ forms a tree. Thus, w.l.o.g. we can choose $\rho$ such that $v_{1}=v_{r-1}$ and there exists a dual triangle $\tau$ such that $\rho=\rho_{1} \rho_{2}$ with $\alpha:=\alpha_{\partial_{0} \rho}=$ $\rho_{1} \tau$ and $\left(\rho_{2}, \tau, \rho_{2}\right)_{\triangleleft}$. Set $\rho_{2}=\tau^{\prime} \rho_{3} \bar{\tau}^{\prime}$, with $\tau^{\prime}$ a direct triangle. Then $L_{\rho}^{h^{\prime}}=L_{\rho_{1}}^{h^{\prime}} L_{\rho_{2}}^{h^{\prime}}=L_{\rho_{1}}^{h^{\prime}} \sum_{k \in G} T_{\tau^{\prime}}^{k} L_{\rho_{3}}^{\bar{k} h^{\prime} k}={ }_{\psi}$ $L_{\rho_{1}}^{h^{\prime}} \sum_{k \in G} T_{\tau^{\prime}}^{k} L_{\bar{\tau}}^{\bar{k}} h^{\prime} k=L_{\rho_{1}}^{h^{\prime}} L_{\tau}^{h^{\prime}}=L_{\alpha}^{h^{\prime}}={ }_{\psi} 1$, where we have used the fact that $\rho_{3} \bar{\tau}$ is a block and (C1) for $\tau, \bar{\tau}$.

(ii) Again the proof is dual to (i) or, alternatively, trivial in terms of Wilson loops.

Corolary 17 Let $\rho=\rho_{1} \rho_{2}$ be a nice strip. Under the same conditions of the previous lemma we have, respectively,

(i) for $h \in H$

$$
L_{\rho_{1}}^{h}={ }_{\psi} \sum_{g \in G} T_{\rho_{1}}^{g} L_{\rho_{2}}^{\bar{g} \bar{h} g}
$$

(ii) for $g \in G$

$$
T_{\rho_{1}}^{g H}={ }_{\psi} T_{\rho_{2}}^{\bar{g} H}
$$

Proof. Apply (B9, B15) to (i) (C3) and (ii) (C4).

For a region $R$ we will understand a collection of faces $f$. We also consider dual regions $R^{*}$, collections of dual faces $v^{*}$. 


\section{Transformation rules}

a. Deformations

Before we define general ribbon deformations, such as the one in Fig. 4, we have to introduce certain simpler ones which are easier to manage in proofs, as the one depicted in Fig. 14. Then simple deformations can be combined together to give the general ones. We say that the ribbons $\rho, \rho^{\prime}$ form a simple deformation, denoted $\left(\rho, \rho^{\prime}\right)_{=}$, if $(i)$ they are open, (ii) they share no triangles, (iii) $\left(\rho, \rho^{\prime}\right)_{\prec \succ}$ and (iv) for any $e \in E_{\rho}^{\triangle}$ we have $\partial_{1} e \in V_{\rho}^{\prime}$. The dual of (iv) is automatically true: for any $e \in E_{\rho^{\prime}}^{\nabla}$ we have $f \in F_{\rho}$ for $f^{*}=\partial_{1} e^{*}$. We will use the notation $F_{\rho, \rho^{\prime}}=F_{\rho}-\left\{f_{s_{0}}, f_{s_{1}}\right\}$ and $V_{\rho, \rho^{\prime}}=V_{\rho^{\prime}}-\left\{v_{s_{0}}, v_{s_{1}}\right\}$, where $s_{i}=\partial_{i} \rho=\partial_{i} \rho^{\prime}$.

Let $R=\left(R_{1}, R_{2}^{*}\right)$ with $R_{1}$ a region and $R_{2}^{*}$ a region of the dual lattice. We introduce the relation between ribbons $\simeq_{R}$ as the minimal equivalence relation such that $\rho_{1}^{\prime} \simeq_{R} \rho_{1}^{\prime}$ if the following conditions are all true: $\rho_{1}=$ $\rho_{2} \rho \rho_{3}, \rho_{1}^{\prime}=\rho_{2} \rho^{\prime} \rho_{3},\left(\rho, \rho^{\prime}\right)=, F_{\rho, \rho^{\prime}} \subset R_{1}$ and $V_{\rho, \rho^{\prime}}^{*} \subset$ $R_{2}^{*}$. Thus, two ribbons are equivalent in the sense of $\simeq_{R}$ if they can be transformed one into the other through simple deformations within $R$. Given a state $|\psi\rangle \in \mathcal{H}_{G}$ and subgroups $H, H^{\prime} \subset G, H$ normal, we define $R_{\psi}^{H . H^{\prime}}=$ $\left(R_{1}, R_{2}^{*}\right)$ with $R_{1}$ the region such that $f \in R_{1}$ iff $B_{f}^{H}={ }_{\psi} 1$ and $R_{2}^{*}$ the dual region such that $v^{*} \in R_{2}^{*}$ iff $A_{v}^{H^{\prime}}={ }_{\psi} 1$. Then we write $\simeq_{\psi}^{H H^{\prime}}$ for $\simeq_{R_{\psi}^{H H^{\prime}}}$.

Proposition 18 Let $|\psi\rangle \in \mathcal{H}_{G}$ and $H, H^{\prime} \subset G$ normal subgroups with $h h^{\prime}=h^{\prime} h$ for any $h \in H, h^{\prime} \in H^{\prime}$. If $\rho$, $\rho^{\prime}$ are ribbons with $\rho \simeq_{\psi}^{H, H^{\prime}} \rho^{\prime}$ then

$$
F_{\rho}^{h^{\prime}, S}={ }_{\psi} F_{\rho^{\prime}}^{h^{\prime}, S}
$$

where $h^{\prime} \in H^{\prime}, S \in G / H$.

Proof. Using (B9) for $\rho=\rho_{1} \rho_{2} \rho_{3}$ we can write

$$
F_{\rho}^{h^{\prime}, g H}=\sum_{l, m \in G} F_{\rho_{1}}^{h^{\prime}, l} F_{\rho_{2}}^{\bar{l} h^{\prime} l, \bar{l} g m H} F_{\rho_{3}}^{\bar{m} \bar{g} h^{\prime} g m, \bar{m}},
$$

and thus it is enough to consider simple deformations $\left(\rho, \rho^{\prime}\right)=$. In that case, we can set $\rho=\tau_{1} \rho_{1} \tau_{1}^{\prime}$ with $\tau_{1}, \tau_{1}^{\prime}$ dual triangles and there exists a ribbon $\rho_{2}$ such that $\rho_{1} \rho_{2}$ is a block and the conditions of lemma 16(ii) are satisfied, so that (C6) applies. But $\left(\rho_{2}, \rho^{\prime}\right)_{\nabla}$, so that using (C2) we get $T_{\rho}^{S}=T_{\rho_{1}}^{S}={ }_{\psi} T_{\rho_{2}}^{\bar{S}}=T_{\rho^{\prime}}^{S}$. We can write $\rho_{2}=\bar{\tau}_{2}^{\prime} \rho_{2}^{\prime} \bar{\tau}_{2}$ and $\rho^{\prime}=\tau_{2} \rho_{3} \tau_{2}^{\prime}$ with $\tau_{2}, \tau_{2}^{\prime}$ direct triangles, and set $\rho_{2}^{\prime \prime}=$ $\bar{\tau}_{1} \rho_{2}^{\prime} \bar{\tau}_{1}^{\prime}$. Then $\left(\rho, \rho_{2}^{\prime \prime}\right) \triangle$ and (C1) applies. Also, $\rho_{2}^{\prime \prime} \rho_{3}$ is a block and the conditions of lemma 16 (i) are satisfied, so that (C5) applies (for $H^{\prime}$ ). Putting everything together we get $L_{\rho}^{h^{\prime}}={ }_{\psi} \sum_{k, l \in G} F_{\rho_{2}^{\prime \prime}}^{l \bar{k} \bar{h}^{\prime} k \bar{l}} T_{\tau_{2}}^{k}={ }_{\psi} \sum_{k \in G} L_{\rho_{2}}^{\bar{k} h^{\prime} k} T_{\tau_{2}}^{k}={ }_{\psi}$ $L_{\rho^{\prime}}^{h^{\prime}}$, where we have used also (B9, B16).

\section{b. Extensions, contractions and rotations}

We also want to consider deformations in which the ends of ribbons are not fixed. Let $Q=\left(Q_{1}, Q_{2}^{*}\right)$ with $Q_{1}, Q_{2} \subset E_{\text {ext }}$. We introduce the relation between ribbons $\asymp_{Q}$ as the minimal equivalence relation such that $\rho \asymp_{Q} \rho^{\prime}$ if $\rho=\rho_{1} \rho^{\prime} \rho_{2}, E_{\rho_{i}}^{\nabla} \subset Q_{1}$ and $E_{\rho_{i}}^{\triangle} \subset Q_{2}$. Thus, two ribbons are equivalent in the sense of $\asymp_{Q}$ if they can be transformed one into the other through extensions or contractions within $Q$. We also introduce an equivalence relation ${\Sigma_{Q}}_{Q}$ for closed ribbons, the minimal such that $\sigma \overline{ }_{Q} \sigma^{\prime}$ if $\left(\sigma, \sigma^{\prime}\right)_{\circ}, E_{\sigma \triangleright \sigma^{\prime}}^{\nabla} \subset Q_{1}$ and $E_{\sigma \triangleright \sigma^{\prime}}^{\triangle} \subset Q_{2}$. Thus, two closed ribbons are equivalent in the sense of $\pm_{Q}$ if they can be transformed one into the other through rotations within $Q$. Given a state $|\psi\rangle \in \mathcal{H}_{G}$ and subgroups $H, H^{\prime} \subset G, H^{\prime}$ normal, we set $Q_{\psi}^{H H^{\prime}}:=\left(Q_{1}, Q_{2}^{*}\right)$ with $Q_{1}$ the collection of edges $e$ with $T_{e}^{H}|\psi\rangle=|\psi\rangle$ and $Q_{2}$ the collection of edges $e^{\prime}$ with $L_{e^{\prime}}^{H^{\prime}}|\psi\rangle=|\psi\rangle$. Then we write $\asymp_{\psi}^{H, H^{\prime}}$ for $\asymp_{Q_{\psi}^{H} H^{\prime}}$ and similarly for $\mp$.

Proposition 19 Let $|\psi\rangle \in \mathcal{H}_{G}$ and $H, H^{\prime} \subset G$ subgroups with $H^{\prime}$ normal.

(i) If $\rho, \rho^{\prime}$ are ribbons with $\rho \asymp_{\psi}^{H H^{\prime}} \rho^{\prime}$ then

$$
\sum_{k \in H} F_{\rho}^{k h^{\prime} \bar{k}, k g H}=\sum_{k \in H} F_{\rho^{\prime}}^{k h^{\prime} \bar{k}, k g H},
$$

where $h^{\prime} \in H^{\prime}, g \in G$.

(ii) If $\sigma, \sigma^{\prime}$ are closed ribbons with $\sigma{ }_{\psi}^{H}{ }_{\psi}^{H^{\prime}} \sigma^{\prime}$ then

$$
\sum_{k \in H} F_{\sigma}^{k h \bar{k}, k g \bar{k}}={ }_{\psi} \sum_{k \in H} F_{\sigma^{\prime}}^{k h \bar{k}, k g \bar{k}} .
$$

where $h, g \in G, h g \bar{h} \bar{g} \in H^{\prime}$.

Proof. (i) It is enough to consider $\rho=\rho^{\prime} \tau$ or $\rho=\tau \rho^{\prime}$ with $\tau$ a triangle and then apply (B9).

(ii) It is enough to consider that $\sigma \triangleright \sigma^{\prime}=\tau$ and then apply (B32).

\section{c. Inversions}

We finally consider other kind of ribbon transformations in which basically ribbons are reversed. As in the other cases, we start introducing suitable relations. For open ribbons $\rho, \rho^{\prime}$ and $R=\left(R_{1}, R_{2}^{*}\right)$ as above, we write $\rho \risingdotseq_{R} \rho^{\prime}$ if $\partial_{0} \rho=\partial_{1} \rho^{\prime}, \partial_{1} \rho=\partial_{0} \rho^{\prime}$ and either (i.a) $\left(\rho, \rho^{\prime}\right)_{\nabla}$ and $V_{\rho}^{*} \subset R_{2}^{*}$ or (i.b) $\left(\rho, \rho^{\prime}\right) \triangle$ and $F_{\rho} \subset R_{1}$. For closed ribbons $\sigma, \sigma^{\prime}$ and a triangle $\tau$, we write $\sigma \doteqdot_{R, \tau} \sigma^{\prime}$ if either (ii.a) $\left(\sigma, \sigma^{\prime}\right)_{\nabla}, \tau$ is dual, $\left(\sigma, \tau, \sigma^{\prime}\right)_{\triangleright}$ and $V_{\rho}^{*} \subset R_{2}^{*}$ or (ii.b) $\left(\sigma, \sigma^{\prime}\right)_{\triangle}, \tau$ is direct, $\left(\sigma, \tau, \sigma^{\prime}\right)_{\triangleleft}$ and $F_{\rho} \subset R_{1}$. For $|\psi\rangle \in \mathcal{H}_{G}$, we write $\risingdotseq_{\psi}^{H H^{\prime}}$ for $\risingdotseq_{R_{\psi}^{H} H^{\prime}}$ and also $\doteqdot_{\psi}^{H H^{\prime}}$ for $\doteqdot_{R_{\psi}^{H H^{\prime}}, \tau}$ if either $\tau$ is dual and $L_{\tau}^{H}={ }_{\psi} 1$ or $\tau$ is direct and $T_{\tau}^{H^{\prime}}={ }_{\psi} 1$. 
Proposition 20 Let $|\psi\rangle \in \mathcal{H}_{G}$ and $H, H^{\prime} \subset G$ normal subgroups with $h h^{\prime}=h^{\prime} h$ for any $h \in H, h^{\prime} \in H^{\prime}$.

(i) If $\rho, \rho^{\prime}$ are open ribbons with $\rho \stackrel{H_{\psi}^{H}, H^{\prime}}{\rho^{\prime}}$ then

$$
F_{\rho}^{h^{\prime}, S}={ }_{\psi} F_{\rho^{\prime}}^{\bar{s} \bar{h}^{\prime} s, \bar{S}},
$$

where $h^{\prime} \in H^{\prime}, s \in S \in G / H$.

(ii) If $\sigma, \sigma^{\prime}$ are closed ribbons with $\sigma \doteqdot{ }_{\psi}^{H} H^{\prime} \sigma^{\prime}$ then

$$
\sum_{k \in H^{\prime}} F_{\rho}^{\bar{k} h^{\prime} k, \bar{k} S k}=\sum_{k \in H^{\prime}} F_{\rho^{\prime}}^{\bar{k} \bar{h}^{\prime} s k, \bar{k} \bar{S} k},
$$

where $h^{\prime} \in H^{\prime}$ and $s \in S \in G / H$ with $s g \bar{s} \bar{g} \in H$.

Proof. (i.a) This case follows from (C2/C5).

(i.b) There exists ribbons $\rho_{i}$ and direct ribbons $\rho_{i}^{\prime}, \rho_{i}^{\prime \prime}$, $i=1,2$, such that $\rho=\rho_{1}^{\prime} \rho_{1} \rho_{1}^{\prime \prime}$ and $\rho=\rho_{2}^{\prime} \rho_{2} \rho_{2}^{\prime \prime}$. Then there exists a direct triangle $\tau$ so that (C1) applies to $\rho_{1}, \rho_{2}$. Moreover, for $s=\partial_{0} \rho_{1}$ we have $\beta_{s}=$ $\tau \rho_{2}^{\prime \prime} \rho_{1}^{\prime}$ and $B_{s}^{H}={ }_{\psi} 1$. Then using also (B9) we have $L_{\rho}^{h^{\prime}}={ }_{\psi} \sum_{m \in G} T_{\rho_{1}^{\prime}}^{m} L_{\rho_{1}}^{\bar{m} h^{\prime} m}={ }_{\psi} \sum_{k, l \in G} F_{\rho_{2}}^{l \bar{k} \bar{h}^{\prime} k \bar{l}, l} T_{\rho_{1}^{\prime} \tau}^{k}={ }_{\psi}$ $\sum_{l \in G} F_{\rho_{2} \rho_{2}^{\prime \prime}}^{l \bar{h}^{\prime}, \bar{l}} T_{\rho_{1}^{\prime} \tau \rho_{2}^{\prime \prime}}^{H}={ }_{\psi} \sum_{l \in G} F_{\rho^{\prime}}^{l \bar{h}^{\prime} \bar{l}, l}$. Together with (C5), this gives (C11).

(ii.a) From (C2) we have $T_{\sigma}^{g}={ }_{\psi} T_{\sigma^{\prime}}^{\bar{g}}$. We can set $\sigma=\tau^{\prime} \rho, \sigma^{\prime}=\rho^{\prime} \bar{\tau}^{\prime}$ with $\tau^{\prime}$ a direct triangle. The strip $\sigma_{0}=\bar{\tau} \rho \tau \rho^{\prime}$ is a nice closed strip, and indeed a block. Then B9|C5 give $L_{\sigma}^{h^{\prime}}={ }_{\psi} \sum_{k \in G} T_{\sigma}^{k} L_{\tau \rho^{\prime} \bar{\tau}}^{\bar{k} \bar{h}^{\prime} k}={ }_{\psi}$ $\sum_{k, l \in G} T_{\sigma}^{k} L_{\tau}^{\bar{k} \bar{h}^{\prime} k} F_{\rho^{\prime}}^{\bar{k} \bar{h}^{\prime} k, l} L_{\bar{\tau}}^{\overline{l k} \bar{h}^{\prime} k l}$. But (C1) implies $L_{\tau}^{g}=$ $\sum_{k \in G} T_{\tau^{\prime}}^{k} L_{\bar{\tau}}^{\bar{k} \bar{g} k}$ for any $g \in G$, and then $L_{\sigma}^{h^{\prime}}={ }_{\psi}$ $\sum_{k \in G} T_{\sigma}^{k} L_{\sigma^{\prime}}^{\bar{k} \bar{h}^{\prime} k}$. The result follows.

(ii.b) From (C1) we have $L_{\rho}^{h^{\prime}}={ }_{\psi} \sum_{k, l \in G} F_{\rho^{\prime}}^{l \bar{k} \bar{h}^{\prime} k \bar{l}, l} T_{\tau}^{k}$. We can set $\sigma=\tau^{\prime} \rho, \sigma^{\prime}=\rho^{\prime} \bar{\tau}^{\prime}$ with $\tau^{\prime}$ a dual triangle. The strip $\sigma_{0}=\bar{\tau} \rho \tau \rho^{\prime}$ is a nice closed strip, and indeed a block. Then (B9|C6) give $T_{\sigma}^{g H}={ }_{\psi} T_{\rho}^{g H}={ }_{\psi} T_{\tau \rho^{\prime} \bar{\tau}}^{\bar{g} H}={ }_{\psi}$ $\sum_{k \in H^{\prime}} T_{\tau}^{k} T_{\sigma^{\prime}}^{\bar{k} \bar{g} k H}$. Using (B13) the result follows.

\section{Deformations in $\mathcal{F}_{\rho}, \mathcal{K}_{\sigma}, \mathcal{J}_{\rho}$ and $\mathcal{K}_{\sigma}^{\prime}$.}

We are now in position to discuss the transformation properties of the ribbon operator algebras introduced in appendix B, We distinguish three cases, which depend on the values of the subgroups $N, M$ that label the Hamiltonian (35).

a. The original Kitaev model: $N=1, M=G$.

In this case we are interested in the algebras $\mathcal{F}_{\rho}$ and $\mathcal{K}_{\sigma}$. As for the first, open ribbons can be deformed so that if $\rho \simeq_{\psi}^{1 G} \rho^{\prime}$ then $F_{\rho}^{h, g}={ }_{\psi} F_{\rho^{\prime}}^{h, g}$. That is, the action of $\mathcal{F}_{\rho}$ is invariant as long as ribbons are deformed without crossing any excitation. They can also be reversed: if $\rho \risingdotseq_{\psi}^{1 G} \rho^{\prime}$ then $F_{\rho}^{h, g}={ }_{\psi} F_{\rho^{\prime}}^{\bar{g} \bar{h} g, \bar{g}}$. Regarding closed ribbons, the action of $\mathcal{K}_{\sigma}$ is invariant under deformations $\left(\simeq_{\psi}^{1 G}\right)$ or rotations $\left(\bar{x}_{\psi}^{G 1}\right)$. Closed ribbon inversions give charge inversion: if $\sigma \doteqdot_{\psi}^{1 G} \sigma^{\prime}$ then $K_{\sigma}^{R C}={ }_{\psi} K_{\sigma^{\prime}}^{\bar{R}^{C} \bar{C}}$, where $R^{C}:=R^{g}$ (as defined in section A 2) with $r_{\bar{C}}=\bar{g} \bar{r}_{C} g$ for some $g \in G$.

\section{b. String tension: $N$ and $M$ normal, $N$ central in $M$.}

In this case we are interested in the algebras $\mathcal{J}_{\rho}$, which gives the domain wall fluxes, and $\mathcal{K}_{\sigma}^{\prime}$, which gives the charges. The action of $\mathcal{J}_{\rho}$ is invariant under deformations which do not cross confined excitations $\left(\simeq_{\psi}^{M N}\right)$, even if ends change as long as they do not cross a domain wall $\left(\asymp_{\psi}^{M N}\right)$. Inversions $\left(\risingdotseq_{\psi}^{M N}\right)$ give domain flux inversion: $(\chi, t)$ goes to $\left(\bar{\chi}^{t}, \bar{t}\right)$. The action of $\mathcal{K}_{\sigma}^{\prime}$ is invariant under deformations $\left(\simeq_{\psi}^{N M}\right)$ or rotations in which the end of $\sigma$ does not cross domain walls $\left(﹎{\psi}^{M N}\right)$. Charge inversion $\left(\stackrel{N}{N}^{N M}\right)$ is as follows: $(R, C)$ goes to $\left(\bar{R}^{C}, \bar{C}\right)$ where $R^{C}:=$ $R^{m}$ with $r_{\bar{C}}=\bar{m} \bar{r}_{C} m$ for some $m \in M$.

\section{c. Domain walls: $N$ normal and abelian.}

In this case we are only interested in domain wall fluxes, that is, in $\mathcal{J}_{\rho}$. Its action is invariant under deformations (those allowed by $\simeq_{*}^{N}$ ), even if ends change as long as they do not cross a domain wall $\left(\asymp_{\psi}^{M N}\right)$. Domain wall flux inversion $\left(\risingdotseq_{\psi}^{N N}\right)$ is as follows: $(R, T)$ goes to $\left(\bar{R}^{T}, \bar{T}\right)$, where $R^{T}:=R^{r_{T} m}$ with $r_{\bar{T}} M=\bar{m} \bar{r}_{T} M$ for some $m \in M$, so that $\mathbf{N}_{\bar{T}}=\bar{m} \bar{r}_{T} \mathbf{N}_{T} r_{T} m$.

\section{Charge types}

The previous results about closed ribbon transformations must be complemented with the following one, which relates proper closed ribbon operators with local vertex and face operators. Let $N, M \subset G$ be normal subgroups in $G$ with $N$ central in $M$, and define for $R \in\left(\mathbf{N}_{C}^{\prime}\right)_{\text {ir }}$ and $C \in(G / N)_{\mathrm{cj}}$

$$
D_{s}^{R C}:=\frac{n_{R}}{\left|\mathbf{N}_{C}^{\prime}\right|} \sum_{D} \sum_{q \in Q_{C}} \sum_{d \in D} \bar{\chi}_{R}(d) A_{s}^{q d \bar{q}} B_{s}^{q r_{C} \bar{q}}
$$

where $D$ runs over $\left(\mathbf{N}_{C}^{\prime}\right)_{\mathrm{cj}}$.

Proposition 21 Let $s$ be a site, $\sigma$ a closed ribbon and $\tau$ a dual triangle with $\beta_{s} \doteqdot V_{\sigma}, \tau$. If $|\psi\rangle \in \mathcal{H}_{G}$ is such that $A_{v}^{M}={ }_{\psi} 1$ for any vertex $v \neq v_{s}$ in $f_{s}$ and $L_{\tau}^{N}={ }_{\psi} 1$ then

$$
K_{\sigma}^{R C}=D_{s}^{R C},
$$

where $R \in\left(\mathbf{N}_{C}^{\prime}\right)_{\mathrm{ir}}, C \in(G / N)_{\mathrm{cj}}$.

Proof. Let $s^{\prime}$ be the second site of $\sigma$, so that $v_{s^{\prime}}=v_{s}$ and $e_{\tau}$ does not belong to $f_{s^{\prime}}$. The states $\left|\psi^{g}\right\rangle:=A_{v}^{M} B_{s^{\prime}}^{g}, \quad g \in G$, are such that 
$\beta_{s} \doteqdot{ }_{\psi^{g}}^{N M} \sigma$. Then with the notation of (C12) we have $\sum_{k \in H^{\prime}} F_{\sigma}^{\bar{k} h^{\prime} k, \bar{k} S k} B_{s^{\prime}}^{g}=\sum_{k \in H^{\prime}} B_{s^{\prime}}^{\bar{k} h^{\prime} k g \bar{k} \bar{h}^{\prime} k} F_{\sigma}^{\bar{k} h^{\prime} k, \bar{k} S k}={ }_{\psi^{g}}$ $\sum_{k \in H^{\prime}} B_{s^{\prime}}^{\bar{k} h^{\prime} k g \bar{k} \bar{h}^{\prime} k} B_{s}^{\bar{k} S k} \quad={ }_{\psi^{g}} \quad \sum_{k \in H^{\prime}} A_{s}^{\bar{k} h^{\prime} k} B_{s}^{\bar{k} S k} B_{s^{\prime}}^{g}$. Since $|\psi\rangle=\sum_{g} B_{s^{\prime}}^{g}\left|\psi^{g}\right\rangle$, the result follows.

\section{APPENDIX D: LOCAL DEGREES OF FREEDOM}

In this appendix we give the details of the results indicated in section (IID). Choose any $C \in(G)_{\mathrm{cj}}$ and two indices $i, i^{\prime}$ and define

$$
|n\rangle:=\left|n ; i, i^{\prime}\right\rangle:=F_{\rho}^{\bar{c}_{i}, \bar{q}_{i} n q_{i^{\prime}}}\left|\psi_{G}\right\rangle,
$$

where $\left|\psi_{G}\right\rangle$ is a ground state of $H_{G}$ (1). Let $V$ be the space with basis $|n\rangle, n \in \mathbf{N}_{C}$. Then there exists an evident isomorphism $p: \mathbf{C}\left[\mathbf{N}_{C}\right] \longrightarrow V$. For $n, n^{\prime} \in \mathbf{N}_{C}$ and $s=\partial_{0} \rho, s^{\prime}=\partial_{1} \rho$, consider the operators

$$
a_{n, n^{\prime}}:=A_{s}^{\bar{q}_{i} n q_{i}} A_{s^{\prime}}^{\bar{q}_{i^{\prime}} n^{\prime} q_{i^{\prime}}} .
$$

They give a representation $a: \mathbf{N}_{C} \times \mathbf{N}_{C} \longrightarrow \mathbf{G L}(V)$ because

$$
a_{n_{1}, n_{2}}|n\rangle=\left|n_{1} n \bar{n}_{2}\right\rangle
$$

so that if $\mathcal{R}: \mathbf{N}_{C} \times \mathbf{N}_{C} \longrightarrow \mathbf{G L}\left(\mathbf{C}\left[\mathbf{N}_{C}\right]\right)$ is the representation of appendix $\mathrm{A}$, we have $a_{n_{1}, n_{2}} p=p \mathcal{R}_{n_{1}, n_{2}}$. This has several consequences. First, if we define in accordance with A15 a basis for $V$ with elements

$$
\left|R ; j j^{\prime}\right\rangle:=\sum_{n \in \mathbf{N}_{C}} \bar{\Gamma}_{R}^{j j^{\prime}}(n)|n\rangle
$$

then in the new basis

$$
a_{n, n^{\prime}}\left|R ; j j^{\prime}\right\rangle=\sum_{k, k^{\prime}=1}^{n_{R}} \Gamma_{R}^{k j}(n) \bar{\Gamma}_{R}^{k^{\prime} j^{\prime}}\left(n^{\prime}\right)\left|R ; k k^{\prime}\right\rangle .
$$

In $\mathbf{C}\left[\mathbf{N}_{C}\right]$ from a19 get

$$
\begin{aligned}
e_{R}^{u v} e_{R^{\prime}}^{j j^{\prime}} e_{R}^{v^{\prime} u^{\prime}} & =\delta_{R, R^{\prime}} \delta_{v, j} \delta_{v^{\prime}, j^{\prime}} e_{R}^{u u^{\prime}}, \\
e_{R} e_{R^{\prime}}^{j j^{\prime}} & =e_{R^{\prime}}^{j j^{\prime}} e_{R}=\delta_{R, R^{\prime}} e_{R}^{j j^{\prime}}
\end{aligned}
$$

which through the isomorphism $p$ give

$$
\begin{gathered}
a_{R}^{u v} a_{\bar{R}}^{\prime} u^{\prime} v^{\prime}\left|R^{\prime} ; j j^{\prime}\right\rangle=\delta_{R, R^{\prime}} \delta_{v, j} \delta_{v^{\prime}, j^{\prime}}\left|R ; u u^{\prime}\right\rangle, \\
a_{R}\left|R^{\prime} ; j j^{\prime}\right\rangle=a_{\bar{R}}^{\prime}\left|R^{\prime} ; j j^{\prime}\right\rangle=\delta_{R, R^{\prime}}\left|R ; j j^{\prime}\right\rangle,
\end{gathered}
$$

where

$$
\begin{array}{r}
a_{R}^{u v}:=\frac{n_{R}}{\left|\mathbf{N}_{C}\right|} \sum_{n \in \mathbf{N}_{C}} \bar{\Gamma}_{R}^{u v}(n) a_{n, 1}, \\
a_{R}^{\prime u v}:=\frac{n_{R}}{\left|\mathbf{N}_{C}\right|} \sum_{n \in \mathbf{N}_{C}} \bar{\Gamma}_{R}^{u v}(n) a_{1, n} \\
a_{R}=\sum_{u=1}^{n_{R}} a_{R}^{u u}, \quad a_{R}^{\prime}=\sum_{u=1}^{n_{R}} a_{R}^{\prime u u} .
\end{array}
$$

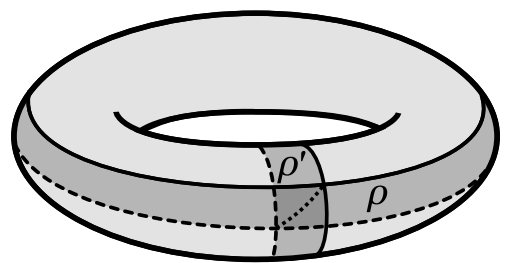

FIG. 15: In a torus we can find a pair of closed ribbons $\sigma, \sigma^{\prime}$ such that they form a crossed joint, $\left(\sigma, \sigma^{\prime}\right)_{+}$. This is not possible in a sphere.

Note that $a_{R}^{u v}, a_{R} \in \mathcal{D}_{s}, a_{R}^{\prime u v}, a_{R}^{\prime} \in \mathcal{D}_{s^{\prime}}$.

Finally, from (B42) we have

$$
B_{s}^{c_{k}} B_{s^{\prime}}^{\bar{c}_{k^{\prime}}}\left|n ; i, i^{\prime}\right\rangle=\delta_{k, i} \delta_{k^{\prime}, i^{\prime}}\left|n ; i, i^{\prime}\right\rangle
$$

and from (B41)

$$
A_{s}^{\bar{q}_{k} q_{i}} A_{s^{\prime}}^{\bar{q}_{k^{\prime}} q_{i^{\prime}}}\left|n ; i, i^{\prime}\right\rangle=\left|n ; k, k^{\prime}\right\rangle .
$$

Note that $\left|R ; j j^{\prime}\right\rangle$ is just a shorthand for (21). Finally, these results must be complemented with proposition 21 .

\section{APPENDIX E: SINGLE-QUASIPARTICLE STATES}

Only in a surface of non-trivial topology can we find two closed ribbons $\sigma, \sigma^{\prime}$ such that $\left(\sigma, \sigma^{\prime}\right)_{+}$, see Fig. 15 . When such ribbons exist, we can construct for any $h, g \in$ $G$ the state

$$
\left|\psi_{h g}\right\rangle:=F_{\sigma}^{h g} L_{\sigma^{\prime}}^{\bar{g}} \prod_{v} A_{v}|\mathbf{1}\rangle
$$

The state $|\psi\rangle$ is not zero, because (B21|B45

$$
L_{\sigma^{\prime}}^{g} L_{\sigma}^{\bar{h}}\left|\psi_{h g}\right\rangle=\prod_{v} A_{v}|\mathbf{1}\rangle .
$$

At most, it can have an excitation at $(v, f)=\partial \sigma=\partial \sigma^{\prime}$. In fact $\mathrm{B} 45$

$$
B_{f}\left|\psi_{h g}\right\rangle=\delta_{g h, h g}\left|\psi_{h g}\right\rangle,
$$

showing that for non-abelian groups single-quasiparticle excitations exist.

\section{APPENDIX F: CONDENSATION}

In this appendix we give the details of the calculations of certain expected values for ribbon operators $\langle F\rangle$ for a ground state of the Hamiltonian (35) for $N \subset M \subset$ $G$ subgroups of $G, N$ normal. Such ground states are characterized by the conditions (36). For $S \subset G, g \in G$ we introduce the notation

$$
\delta_{g, S}:=\delta_{g S, S}
$$


Proposition 22 Let $h, g \in G, n \in N$ and $|\psi\rangle,|\psi\rangle^{\prime} \in \mathcal{H}_{G}$ satisfy (36).

(i) For an arbitrary ribbon $\rho$

$$
\begin{aligned}
F_{\rho}^{h, g}|\psi\rangle & =\delta_{g, M} F_{\rho}^{h n, g}|\psi\rangle, \\
\left\langle\psi^{\prime}\left|F_{\rho}^{h, g}\right| \psi\right\rangle & =\delta_{h, M}\left\langle\psi^{\prime}\left|F_{\rho}^{h, g n}\right| \psi\right\rangle .
\end{aligned}
$$

(ii) If $\rho$ is an open ribbon

$$
\begin{aligned}
F_{\rho}^{N M}|\psi\rangle & =|\psi\rangle, \\
\left\langle\psi^{\prime}\left|F_{\rho}^{h, g}\right| \psi\right\rangle & =\delta_{h, N} \delta_{g, M} \frac{1}{|M|}\left\langle\psi^{\prime} \mid \psi\right\rangle .
\end{aligned}
$$

(iii) If $\sigma$ is a boundary ribbon

$$
\begin{aligned}
F_{\sigma}^{M N}|\psi\rangle & =|\psi\rangle, \\
\left\langle\psi^{\prime}\left|F_{\sigma}^{h, g}\right| \psi\right\rangle & =\delta_{h, M} \delta_{g, N} \frac{1}{|N|}\left\langle\psi^{\prime} \mid \psi\right\rangle .
\end{aligned}
$$

Proof. (i) If $\rho$ is a triangle this is a direct consequence of the identities $L_{\tau}^{n} L_{\tau}^{N}=L_{\tau}^{N}, T_{\tau}^{g} T_{\tau}^{M}=\delta_{g, M} T_{\tau}^{g} T_{\tau}^{M}$, $T_{\tau}^{M} L_{\tau}^{h} T_{\tau}^{M}=\delta_{h, M} T_{\tau}^{M} L_{\tau}^{h} T_{\tau}^{M}$ and $L_{\tau}^{N} T_{\tau}^{g} L_{\tau}^{N}=L_{\tau}^{N} T_{\tau}^{g n} L_{\tau}^{N}$. For general ribbons, just apply (B9).

(ii) From (i) we get $F_{\sigma}^{N M}|\psi\rangle=F_{\sigma}^{1 G}|\psi\rangle=|\psi\rangle$ using (B16). Let $s_{i}=\partial_{i} \rho$ and set $\langle\cdot\rangle:=\left\langle\psi^{\prime}|\cdot| \psi\right\rangle$. Then from (B12, 36) we have $\left\langle F_{\rho}^{h, g}\right\rangle=\left\langle B_{s_{0}}^{N} F_{\rho}^{h, g}\right\rangle=\left\langle F_{\rho}^{h, g m} B_{s_{0}}^{N h}\right\rangle=$ $\delta_{h, N}\left\langle F_{\rho}^{h, g m}\right\rangle$ and for $m \in M$ from (B41, 36) we have $\left\langle F_{\rho}^{h, g}\right\rangle=\left\langle F_{\rho}^{h, g} A_{s_{1}}^{m}\right\rangle=\left\langle A_{s_{1}}^{m} F_{\rho}^{h, g m}\right\rangle=\left\langle F_{\rho}^{h, g m}\right\rangle$. Thus $\left\langle F_{\sigma}^{h, g}\right\rangle=\delta_{g, M} \delta_{h, N}\left\langle F_{\sigma}^{1,1}\right\rangle$ and the result follows since $\left\langle F_{\rho}^{1, M}\right\rangle=\left\langle F_{\rho}^{1, G}\right\rangle=\langle 1\rangle$.

(iii) Using the notation of appendix $\mathbb{C} p_{\sigma}$ encloses a disc $R \subset R_{\psi}^{N}$ so that $F_{\sigma}^{M N}|\psi\rangle=|\psi\rangle$. Also, $L_{\sigma}^{m}|\psi\rangle=|\psi\rangle$ for any $m \in M$. To check this, suppose for example that the edges $E_{\rho}^{\triangle}$ lie outside $R$ and choose for each vertex $v$ in $R$ a ribbon $\rho_{v}$ with $p_{\rho_{v}}$ a path inside $R$ from $v_{0}=v_{\partial_{0} \rho}$ to $v$. If we set $A_{\rho}^{m}=A_{v_{0}}^{m} \prod_{v \neq v_{0}} \sum_{k} T_{\rho_{v}}^{k} A_{v}^{\bar{k} m k}$, with the product running over all vertices in $R$, one can check that $|\psi\rangle=A^{m}|\psi\rangle=L_{\sigma}^{m}|\psi\rangle$. The other case is similar. Thus, for $m \in M$ we get $\left\langle F_{\sigma}^{h, g}\right\rangle=\delta_{g, N}\left\langle F_{\sigma}^{h m, g}\right\rangle$, so that $\left\langle F_{\sigma}^{h, g}\right\rangle=\delta_{g, N} \delta_{h, M}\left\langle F_{\sigma}^{1,1}\right\rangle$. The result follows since $\left\langle F_{\rho}^{1, N}\right\rangle=\left\langle F_{\rho}^{1, G}\right\rangle=\langle 1\rangle$.

A state satisfying (F4] F6 for all open ribbons $\rho$ and boundary ribbons $\sigma$ cannot contain vertex, face or edge excitations. Therefore, these conditions characterize ground states.

We proceed to check (49), the derivation of (56) is similar. From (B70, F7) we get

$$
\left\langle K_{\sigma}^{D C}\right\rangle=\frac{|C \cap N|}{|N||G|} \sum_{g \in G}|D \cap \bar{g} M g|,
$$

where $D \in\left(\mathbf{N}_{C}\right)_{\mathrm{cj}}, C \in(G)_{\mathrm{cj}}$. This together with B75) gives (49) because if $e_{M} \uparrow$ is the induced representation in $G$ of the identity representation in $M$

$$
\chi_{e_{M} \uparrow}(g)=\frac{1}{|M|} \sum_{k \in G} \delta_{g, \bar{k} M k} .
$$

As for (55), from (F5) we have

$$
|M|\left\langle\sum_{n \in \mathbf{N}_{C}} \Gamma_{R}^{j j^{\prime}}(n) F_{\rho}^{\bar{c}_{i}, \bar{q}_{i} n q_{i}^{\prime}}\right\rangle=\delta_{c_{i}, N} \sum_{n \in M_{c}^{i, i^{\prime}}} \Gamma_{R}^{j j^{\prime}}(n),
$$

where $M_{c}^{i, i^{\prime}}:=\mathbf{N}_{C} \cap q_{i} M \bar{q}_{i}^{\prime}$. If $M_{c}^{i, i^{\prime}}$ is empty, we are done. Else, $M_{c}^{i, i^{\prime}}=M_{c}^{i, i} s$ for some $s \in \mathbf{N}_{C}$, so that $\sum_{n \in M_{c}^{i, i^{\prime}}} \Gamma_{R}(n)=\sum_{n \in M_{c}^{i, i}} \Gamma_{R}(n) \Gamma_{R}(s)$. But [63] $\sum_{n \in M_{c}^{i, i}} \Gamma_{R}(n)=0$ if $\left(\chi_{R}, 1\right)_{M_{C}^{i, i}}=0$.
[1] X.-G. Wen. Quantum Field Theory of Many-body Systems, Oxford University Press, (2004).

[2] X.-G. Wen and Q. Niu, Phys. Rev. B 41, 9377 (1990).

[3] X.-G. Wen, Int. J. Mod. Phys. B 4, 239 (1990).

[4] X.-G. Wen, Int. J. Mod. Phys. B 6, 1711 (1992).

[5] X.-G. Wen, Phys. Rev. B 44, 2664 (1991).

[6] J. Fröhlich and T. Kerler, Nucl. Phys. B 354, 369 (1991).

[7] D.S. Rokshar and S.A. Kivelson, Phys. Rev. Lett. 61, 2376 (1988).

[8] N. Read and B. Chakraborty, Phys. Rev. B 40, 7133 (1989).

[9] R. Moessner and S.L. Sondhi, Phys. Rev. Lett. 86, 1881 (2001).

[10] E. Ardonne, P. Fendley and E. Fradkin, Annals of Phys. 310, 493 (2004).

[11] V. Kalmeyer and R.B. Laughlin, Phys. Rev. Lett. 59, 2095 (1987).

[12] X. G. Wen, F. Wilczek, and A. Zee, Phys. Rev. B 39, 11413 (1989).

[13] N. Read and S. Sachdev, Phys. Rev. Lett. 66, 1773 (1991).
[14] T. Senthil and M.P. Fisher, Phys. Rev. Lett. 86, 292 (2001).

[15] X.-G. Wen, Phys. Rev. B 65, 165113 (2002).

[16] S. Sachdev and K. Park, Annals of Phys. 298, 58 (2002).

[17] L. Balents, M. P. A. Fisher, and S. M. Girvin Phys. Rev. B 65, 224412 (2002).

[18] A.Yu. Kitaev, Annals Phys. 303, 2 (2003), quant-ph/9707021.

[19] M. Levin and X.-G. Wen, Phys. Rev. B 67, 245316 (2003).

[20] M. Levin and X.-G. Wen, Phys. Rev. B 71, 045110 (2005).

[21] H. Bombin and M.A. Martin-Delgado Phys. Rev. B 75, 075103 (2007); cond-mat/0607736

[22] F.A. Bais, Nucl. Phys. B170, 3 (1980).

[23] F.A. Bais, Phys. Lett. B98, 437 (1981).

[24] L.M. Krauss and F. Wilczek, Phys. Rev. Lett. 62, 1221 (1989).

[25] J. Preskill and L.M. Krauss, Nucl. Phys. B341, 50 (1990).

[26] M. de Wild Propitius and F.A. Bais, in Particles and Fields, edited by G. Semenoff and L. Vinet, CRM Series 
in Mathematical Physics (Springer-Verlag, New York, 1998), p. 353.

[27] F. A. Bais, B. J. Schroers, and J. K. Slingerland, Phys. Rev. Lett. 89, 181601 (2002); arXiv:hep-th/0205117.

[28] F. A. Bais, B. J. Schroers, and J. K. Slingerland, JHEP 0305 (2003) 068; arXiv:hep-th/0205114

[29] J. K. Slingerland and F. A. Bais, Nucl. Phys. B612, 229 (2001); cond-mat/0104035.

[30] E. Dennis, A. Kitaev, A. Landahl, J. Preskill, J. Math. Phys. 43, 4452-4505 (2002).

[31] S. B. Bravyi, A. Yu. Kitaev, quant-ph/9811052

[32] R. W. Ogburn and J. Preskill, Lecture Notes in Computer Science 1509, 341-356, (1999).

[33] Michael H. Freedman, Alexei Kitaev, Zhenghan Wang, Commun.Math.Phys. 227 587-603, (2002).

[34] M. Freedman, M. Larsen, Z. Wang, Comm.Math. Phys. 227 605-622, (2002).

[35] M. H. Freedman, A. Kitaev, M. J. Larsen, Z. Wang, Bull. Amer. Math. Soc. 40 31-38, (2003); quant-ph/0101025

[36] C. Mochon, Phys. Rev. A 69, 032306 (2004); arXiv:quant-ph/0306063.

[37] L.S. Georgiev, arXiv:hep-th/0611340v2.

[38] S. Das Sarma, M. Freedman, C. Nayak, S.H. Simon, A. Stern arXiv:0707.1889

[39] H. Bombin and M. A. Martin-Delgado; Phys. Rev. Lett. 97, 180501 (2006); quant-ph/0605138

[40] H. Bombin and M.A. Martin-Delgado; Phys. Rev. Lett. 98, 160502 (2007); quant-ph/0610024

[41] R. Raussendorf, J. Harrington1 and K. Goyal, New J. Phys. 9, 199 (2007); arXiv:quant-ph/0703143 1.

[42] A. Galindo and M.A. Martin-Delgado, Rev.Mod.Phys. 74347 (2002); quant-ph/0112105.

[43] H. Bombin and M.A. Martin-Delgado; J. Math. Phys. 48, 052105 (2007); quant-ph/0605094

[44] H. Bombin and M.A. Martin-Delgado; Phys. Rev. A 76,
012305 (2007); quant-ph/0703272.

[45] F. Verstraete, M.A. Martin-Delgado, J.I. Cirac, Phys. Rev. Lett. 92, 087201 (2004).

[46] W. Dur, H.J. Briegel, Rep. Prog. Phys. 70, 1381 (2007); arXiv:0705.4165

[47] E. Rico and H.J. Briegel, arXiv:0710.2349.

[48] H. Katsura, T. Hirano, V.E. Korepin; arXiv:0711.3882.

[49] S. Yang, D.L. Zhou, C.P. Sun, arXiv:0708.0676, (2007).

[50] A. Hamma, D. A. Lidar; arXiv:quant-ph/0607145

[51] L.-M. Duan, E. Demler, M. D. Lukin, Phys. Rev. Lett. 91, 090402 (2003), cond-mat/0210564.

[52] A. Micheli, G.K. Brennen, P. Zoller, quant-ph/0512222.

[53] J. Du, J. Zhu, M. Shi, X. Peng, D. Suter, Phys. Rev. A 76, 042121 (2007); arXiv:0705.3566.

[54] J. J. Garcia-Ripoll, M. A. Martin-Delgado, J. I. Cirac, Phys. Rev. Lett. 93, 250405 (2004).

[55] L.B. Ioffe, M.V. Feigel'man, A. Ioselevich, D. Ivanov, M. Troyer, and G. Blatter, Nature 415, 503 (2002).

[56] B. Doucot, L.B. Ioffe, J. Vidal, Phys.Rev. B69 214501 (2004); arXiv:cond-mat/0302104

[57] A.F. Albuquerque, H.G. Katzgraber, M. Troyer, G. Blatter, arXiv:0708.0191

[58] R. Dijkgraaf, V. Pasquier, and P. Roche, Nucl. Phys. (Proc. Suppl.) B18, 60 (1990).

[59] F.A. Bais, P. van Driel, and M. de Wild Propitius, Phys. Lett. B 280, 63 (1992).

[60] H. Bombin, M.A. Martin-Delgado, arXiv:0705.0007

[61] M. B. Hastings, Xiao-Gang Wen, Phys.Rev. B72 (2005) 045141;

[62] R. Alicki, M. Fannes, M. Horodecki, J. Phys. A: Math. Theor. 40, 6451 (2007); arXiv:quant-ph/0702102

[63] J.-P. Serre, "Linear Representations of Finite Groups", Springer-Verlag, New York, 1977 